\title{
A review of wetting versus adsorption, complexions, and related phenomena: the rosetta stone of wetting
}

\author{
Wayne D. Kaplan · Dominique Chatain • \\ Paul Wynblatt $\cdot$ W. Craig Carter
}

Received: 11 May 2013/Accepted: 22 May 2013/Published online: 27 June 2013

(C) The Author(s) 2013. This article is published with open access at Springerlink.com

\begin{abstract}
This paper reviews the fundamental concepts and the terminology of wetting. In particular, it focuses on high temperature wetting phenomena of primary interest to materials scientists. We have chosen to split this review into two sections: one related to macroscopic (continuum) definitions and the other to a microscopic (or atomistic) approach, where the role of chemistry and structure of interfaces and free surfaces on wetting phenomena are addressed. A great deal of attention has been placed on thermodynamics. This allows clarification of many important features, including the state of equilibrium between phases, the kinetics of equilibration, triple lines, hysteresis, adsorption (segregation) and the concept of complexions, intergranular films, prewetting, bulk phase transitions versus "interface transitions", liquid versus solid wetting, and wetting versus dewetting.
\end{abstract}

W. D. Kaplan $(\bowtie)$

Department of Materials Science \& Engineering, TechnionIsrael Institute of Technology, 32000 Haifa, Israel

e-mail: kaplan@tx.technion.ac.il

D. Chatain

CNRS, CINaM UMR 7325, Aix Marseille Université,

13288 Marseille, France

P. Wynblatt

Department of Materials Science \& Engineering, Carnegie

Mellon University, Pittsburgh, PA 15213, USA

W. C. Carter

Department of Materials Science \& Engineering, MIT, Cambridge, MA, USA

\section{Introduction}

High temperature capillarity is an important scientific and technological field of research. The degree by which a liquid wets a solid is an important technological parameter for processes such as joining [1-6], solidification [7-9], and composite processing [10-14]. While wetting is a measure of the "energy" of interfaces between bulk phases, and thus a parameter associated with equilibrium thermodynamics, the rate by which a liquid spreads in contact area with a solid is equally important for technological processes [15-17]. Fundamentally, wetting depends on the chemical content and atomistic structure of the bulk phases and the interface itself. This review first attempts to identify phenomena related to wetting between phases, and then proceeds to describe how these phenomena may be modified by the presence of adsorption (segregation). This includes the role of anisotropy of crystalline materials in wetting, and the heterogeneity and roughness of surfaces, and we clearly separate between equilibrium (wetting) and kinetics (spreading).

While solid-liquid interfaces are often important for materials processing, it is the solid-solid interface which frequently determines the mechanical and functional properties of the final material system. It is the solid-solid interfacial energy which defines the nominal energy required to fracture a solid at a join, ignoring irreversible processes and deformation [18-20], and thus measuring and decreasing solid-solid interface energy offers an engineering approach for the optimization of mechanical properties via fundamental interface science [21, 22]. As such we have explicitly reviewed the concept of solid-solid wetting, how solid-solid interfacial energy and the thermodynamic work of adhesion can be experimentally measured, and how the anisotropy of crystalline materials must be taken into account. 
Finally, we have reviewed the fundamentals of adsorption at the thermodynamic, or macroscopic, scale, and why adsorption must be considered in the analysis of both wetting and wetting transitions. Adsorption has also been considered at the level of the local atomistic structure, first with regard to excess distribution and then by using the concept of interface complexions. It is our hope that this review will demonstrate to the reader that fully understanding wetting phenomena requires the concept of complexions, and that including complexions offers the possibility to merge continuum and atomistic approaches to interface science.

\section{Interfaces and their energies}

In what follows, the term interface is used in a generic sense to indicate any region of a material that separates two distinguishable bulk phases. Typically, the interface can be treated as a thin slab in which the features which distinguish the two bulk phases vary from one bulk material to another, or it can be replaced by a mathematical plane. This general definition of the term interface naturally includes the surface of a solid in contact with a gas phase, and the boundary between two grains of the same phase, but with differing orientations (a grain boundary). The term 'surface' is reserved for the subset of interfaces between condensed phases and their equilibrium vapor. As is now wellknown, if at least one of the two phases separated by an interface is crystalline, then the energy ${ }^{1}$ of that interface, $\gamma$, may be anisotropic, i.e., it may depend on the crystallographic orientation of the interface with respect to the crystalline phase(s), and the misorientation of the abutting phases if they are both crystalline. To simplify the presentation, we will treat wetting from the simplest case and progress to more complex systems.

\section{Macroscopic wetting of a liquid on a rigid solid substrate}

Wetting phenomena involve interactions among three separated volumes, which abut three interfaces and meet at a triple line. The Young contact angle, $\theta_{\mathrm{Y}}$, of a wetting phase on a rigid substrate (or wetted phase) is related to the interfacial energies by the Young equation, written here for

\footnotetext{
$1 \gamma$ is technically an interfacial energy density, or free energy per unit area. It is traditional to call $\gamma$ the interfacial (or surface) energy, which may be confused with the total interfacial energy $\int \gamma \mathrm{d} A$. In the bulk of our paper we will discuss means by which $\gamma$ can be changed, and if we refer to $\int \gamma \mathrm{d} A$ we will specifically state this.
}

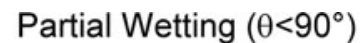

Partial Wetting $\left(\theta>90^{\circ}\right)$
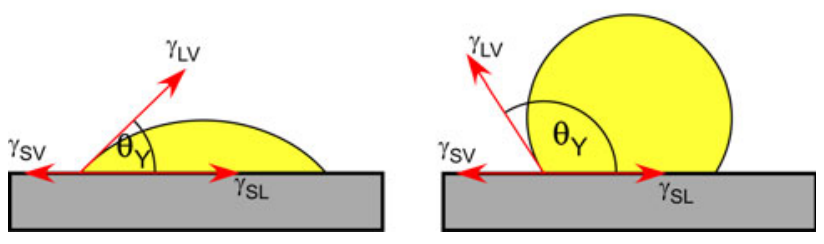

Fig. 1 Young, or equilibrium, or intrinsic contact angle and interfacial energies

water on glass

(a)

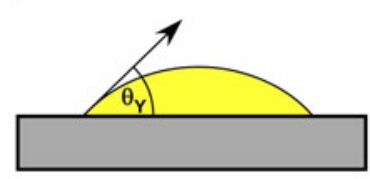

(b)

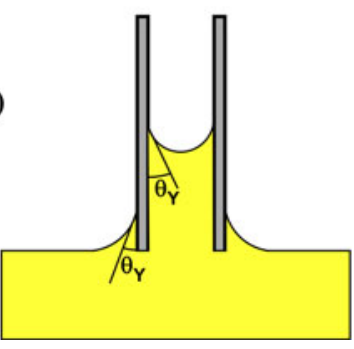

Fig. 2 Examples of a sessile drops and $\mathbf{b}$ capillary rise of water and depression of mercury on a same glass surface. The contact angle for a given three-phase system does not change with the macroscopic shape of the solid

a liquid wetting phase $(L)$ on a solid substrate $(S)$ in a vapor phase $(V)$ :

$\cos \theta_{\mathrm{Y}}=\frac{\gamma_{\mathrm{SV}}-\gamma_{\mathrm{SL}}}{\gamma_{\mathrm{LV}}}$

where $\gamma_{i j}$ are the energies of the three interfaces $i j$, and $i$ and $j$ are the phases that coexist at equilibrium. As such, at equilibrium $\theta_{\mathrm{Y}}$ reflects the relative interfacial energies of the system.

This equation corresponds to the vector equilibrium obtained by representing the energies of the three interfaces at the triple line as interfacial tensions projected onto the solid plane (see Fig. 1) ${ }^{2}$ [23]. It can also be derived from the values of the interfacial energy densities. Young's equation will apply only if these interfacial energies are isotropic.

At the macroscopic scale, a liquid on a flat horizontal solid surface (or substrate) adopts a shape generally referred to as a sessile drop (see Fig. 2a). The Young contact angle, $\theta_{\mathrm{Y}}$, at the solid-liquid-vapor triple line, must

\footnotetext{
2 The surface tension may differ in value from the surface energy. Technically, the surface tension is a tensor quantity [23].
} 
be measured in a plane perpendicular to both the substrate and the triple line.

Under the influence of gravity, the shape of the drop changes as the result of an equilibrium between competing forces due to capillary pressure (under which the drop would adopt the shape of a spherical cap) and hydrostatic pressure (under which the drop would spread and flatten), but the equilibrium contact angle $\theta_{\mathrm{Y}}$ does not change due to the influence of gravity. The capillary length, $L_{\mathrm{c}}$, is a characteristic length scale for a liquid surface subject to both pressures:

$L_{\mathrm{c}}=\sqrt{\frac{\gamma_{\mathrm{LV}}}{\Delta \rho g}}$

where $\Delta \rho$ is the difference in density between the two fluids coexisting at the surface, and $g$ is the acceleration due to gravity. Drops smaller than $L_{\mathrm{c}}$ will remain spherical, whereas larger drops will flatten.

For a given solid-liquid-vapor system, the Young contact angle does not depend on the macroscopic shape of the solid if the solid is smoothly curved. For example, when the solid is in the shape of a small vertical tube, the contact angles inside and outside the tube are identical to that of a sessile drop of the same liquid on a planar substrate of the same solid. If the contact angle is less than $90^{\circ}$ (greater than $90^{\circ}$ ), then the liquid on the interior of the tube will rise (be depressed) as shown in Fig. 2b; this is the phenomenon of capillary rise or depression. The length of the rise is set by the contact angle on the interior of the cylinder, by $L_{\mathrm{c}}$, and by the difference in liquid curvature between the inside and outside liquid surfaces (the curvature difference supports the hydrostatic pressure created by the capillary rise: see Fig. 2b). Cases for a surface which is not smooth will be dealt with in subsequent sections. Again, the height of the liquid in the tube results from a balance between the capillary and hydrostatic pressures.

In addition to the contact angle, the thermodynamic work of adhesion $\left(W_{\mathrm{ad}}\right)$ is often used to compare the relative interfacial and surface energies of a particular system. $W_{\text {ad }}$ is the work per unit area necessary to separate an interface of interfacial energy $\gamma_{\mathrm{SL}}$ into two equilibrated (i.e., including any adjustments of surface energy due to adsorption or reconstruction) surfaces of energies $\gamma_{\mathrm{LV}}$ and $\gamma_{\mathrm{SV}}$ :

$W_{\mathrm{ad}}=\left(\gamma_{\mathrm{LV}}+\gamma_{\mathrm{SV}}\right)-\gamma_{\mathrm{SL}}$

It is important to differentiate between the thermodynamic work of adhesion and the work of separation. The work of separation is often used in fracture analysis, or in atomistic simulations, to define the difference in energy between an equilibrated interface and the two surfaces created immediately after the interface has been separated (i.e., before the newly created surfaces have reached equilibrium).
Since the surface energy is a minimum at equilibrium, the work of separation is larger than the work of adhesion. If all the interfaces are isotropic, then by combining Eq. (3) with Young's equation (1), $W_{\text {ad }}$ can be expressed as a function of the contact angle (the Young-Dupré equation):

$W_{\mathrm{ad}}=\gamma_{\mathrm{LV}}(1+\cos \theta)$

This is a very useful relationship since it expresses $W_{\mathrm{ad}}$ in terms of two experimentally measurable quantities in solid-liquid-vapor systems: $\gamma_{\mathrm{LV}}$ and $\theta_{\mathrm{Y}}$.

In principle, contact angles can have any value between $0^{\circ}$ and $180^{\circ}$. Materials scientists working with inorganic materials at high temperature tend to distinguish between two types of systems, "good wetting" systems where $\theta_{\mathrm{Y}}<90^{\circ}$, and "bad wetting" systems, $\theta_{\mathrm{Y}}>90^{\circ}$. This nomenclature is related to the ability of a liquid to spontaneously rise within an ideal vertical capillary tube when $\theta_{\mathrm{Y}}<90^{\circ}$. For an isotropic system consisting of a droplet trapped between two flat coplanar plates, the capillary force between the plates is only negative (pulling the plates towards each other) when $\theta_{\mathrm{Y}}<=90^{\circ}$. When $\theta_{\mathrm{Y}}>90^{\circ}$, the capillary force can have either sign and the plates have an equilibrium separation at zero force (see Cannon and Carter [24, 25] for a derivation of this phenomenon as well as a variational formulation of the equilibrium shapes, and the boundary conditions to Euler's equation which must be satisfied by the Young-Dupré equation). We prefer the use of the nomenclature: "Partial wetting" for any contact angle between $0^{\circ}$ and $180^{\circ}$ (see Fig. 1).

Instead of partial wetting, scientists who deal with organic systems use the terms "wetting" and "non-wetting" to describe systems which display contact angles that are zero or positive, respectively. To avoid confusion, we will use the terminology "complete" or "perfect" wetting when the contact angle is zero, and "non-wetting" when the contact angle is $180^{\circ}$; thus the limiting conditions of partial wetting are complete (or perfect) wetting and non-wetting.

The observation of a continuous layer at an interface does not necessarily imply perfect wetting. Such observed layers may not correspond to an equilibrium phase, but rather to an interfacial layer which minimizes the total free energy by local adjustment of structure, density, and/or chemical composition. The name "complexions" has recently been assigned to such layers. This is an important issue which will be addressed in detail in sections "Microscopic scale and adsorption" and "Complexions". Complete wetting requires that the interfacial layer be an equilibrium bulk phase that coexists with its abutting phases (or phase). Thus, in a given system, it is essential to verify that a wetting phase conforms to the coexistence conditions of the corresponding phase diagram. In addition, the relevant phase diagram must include any species that are present in the interfacial regions, including those present in the vapor phase, because even at very low partial 
pressures, certain species may be adsorbed at the surfaces and interfaces (such as oxygen at metallic and oxide surfaces/interfaces). See "Panel 1".

When the contact angle is $180^{\circ}$, wetting is "null". In this case, it is the vapor phase that completely wets the solid at coexistence with the liquid. Apparent contact angles close to $180^{\circ}$ can be obtained when the morphology of the solid surface is specifically designed to reduce the actual contact between the liquid phase and the entire area of the solid surface. This "super-hygrophobic" 3 phenomenon [26], originally referred to as "composite wetting" [27] and recently renamed the "lotus effect", will be described in greater detail in the next section.

\section{Panel 1: influence of oxygen adsorption on copper surfaces}

The influence of oxygen adsorption on copper surfaces is used here as an example of the effects of adsorption on properties. Figure P1-1 shows the change in surface energy of liquid copper as a function of $\sqrt{ }\left(\mathrm{PO}_{2}\right)$ where $P\left(\mathrm{O}_{2}\right)$ is the oxygen partial pressure $(\mathrm{atm})$. The figure shows the significant decrease in surface energy (by about $40 \%$ ) that can be produced by exposure to an environment that contains a relatively low oxygen partial pressure.

Figure P1-2 illustrates the influence of equilibration of a solid copper crystal in two different oxygen partial pressures. The micrographs on the left shows a crystal equilibrated at $1253 \mathrm{~K}$ in an oxygen partial pressure of $10^{-18} \mathrm{~atm}$. Under these conditions oxygen adsorption is negligible, and the copper crystal displays an equilibrium crystal shape (ECS) that is essentially identical to that of pure copper at this temperature [28]. This ECS consists of small $\{111\}$ and $\{100\}$ facets, with all possible surface orientations present, so that the facets merge smoothly into the curved portions of the ECS. The photomicrograph on the right corresponds to equilibration at $1253 \mathrm{~K}$ in an oxygen partial pressure of $10^{-12} \mathrm{~atm}$. Here, the ECS also displays $\{111\}$ and $\{100\}$ facets, but in contrast to the picture on the left, some surface orientations are missing. As a result, the

\footnotetext{
3 The reader will more often find in the literature use of the term 'hydrophobic' (or 'hydrophilic') rather than hygrophobic (or hygrophilic). Hydrophobic (or hydrophilic) necessarily deals with very specific case of wetting of a surface by water whereas the terms hygrophobic or hygrophilic refer to general liquids. The reader is referred to the recent publication by Marmur for applications of terminology in wetting [26].
}

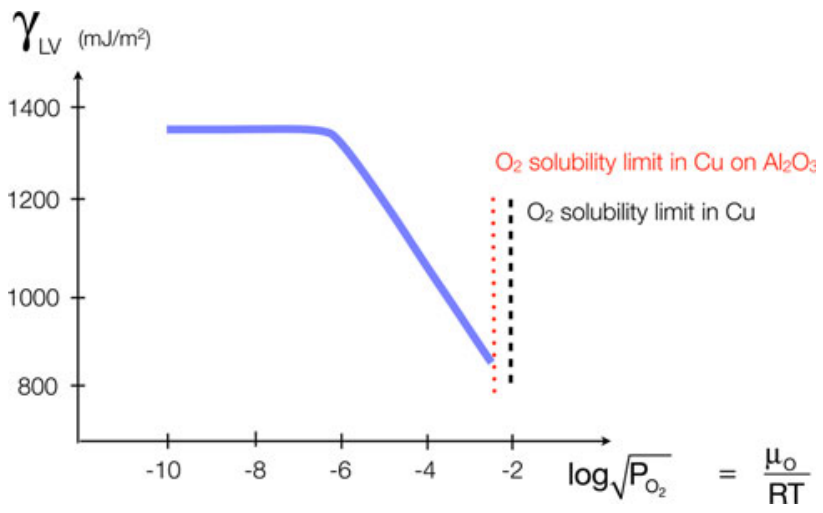

Fig. P1-1 Variation of the surface energy of liquid copper with oxygen partial pressure at $1373 \mathrm{~K}$ [150]

(100)

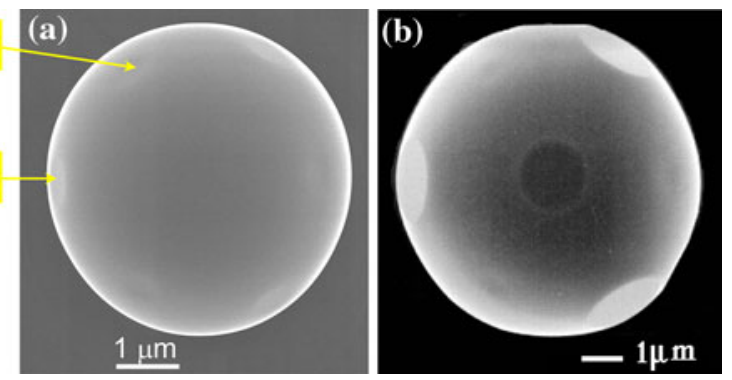

Fig. P1-2 Micrographs of copper crystals equilibrated at $1253 \mathrm{~K}$ $(0.9 \mathrm{Tm})$ in $\mathrm{H}_{2} / \mathrm{H}_{2} \mathrm{O}$ mixtures corresponding to oxygen partial pressures of either $10^{-18}$ atm $(\mathbf{a})$, or $10^{-12}$ atm (b)

facets have sharp edges, and are therefore more easily identified.

There have been relatively few reports on the changes in the ECS by adsorption effects. However, there is one study where the ECS of $\mathrm{Pb}$ has been investigated as a function of temperature for two different bulk compositions of ternary $\mathrm{Pb}-\mathrm{Bi}-\mathrm{Ni}$ alloys [29].

\section{Wetting on heterogeneous substrates}

An actual solid surface is often macroscopically rough and spotted with chemical heterogeneities. This is one of the main, and often forgotten, origins of scatter in contact angle data. When a wetting experiment is performed with a liquid drop of a size that is much larger than the surface defects of the substrate, the measured macroscopic contact angle depends not only on the wetting of the liquid on these defects but also on the path followed by the triple line of the drop prior to the contact angle measurement [30]. Understanding the factors that control the position of the triple line on an imperfect substrate is important. Indeed, micro-patterning of surfaces with geometric and/or chemical features can be used to produce contact angles that cannot be inferred from the Young equation. This may be 
referred to as "apparent wetting". In the following, explanations are provided through some simple examples.

Wetting on rough surfaces

The wetting of a drop on rough surfaces with simple geometries has been addressed theoretically by Huh and Mason [31]. A randomly rough substrate resembles a landscape of hills and valleys on which the contact angle corresponds locally to the intrinsic (or Young) contact angle of the surface, $\theta_{\mathrm{Y}}$. The deviation of the local tilt angle of the substrate from the average plane of the solid surface is defined as $\delta$. Figure 3 shows a schematic of a 2D saw-tooth roughness where the slopes are $+\delta$ and $-\delta(\delta>0)$. On this simple model of $2 \mathrm{D}$ roughness the macroscopic contact angle, measured at the intersection of the macroscopic shape of the 2D drop by the average plane of the substrate, can take on any value between the minimum and the maximum local angles of $\theta_{\mathrm{Y}}-\delta$ and $\theta_{\mathrm{Y}}+\delta$, respectively. These extreme angles can be achieved by moving the triple line of the drop inwards or outwards, and are referred to as the minimum receding and the maximum advancing contact angles. The difference between these two angles defines the maximum wetting hysteresis, which is equal to $2 \delta$.

Within the range of hysteresis, there is one value of contact angle which corresponds to a minimum in the total interfacial energy of a sessile drop on a microscopically rough substrate; it is known as the Wenzel contact angle [32]. It takes into account the increase in the areas of the solid/liquid interface due to the roughness. If $(1+K)$ is the ratio of the actual to the geometric solid/liquid interface, the Wenzel equilibrium contact angle, $\theta_{\mathrm{W}}$, is written as follows:

$\cos \theta_{\mathrm{w}}=(1+K) \frac{\gamma_{\mathrm{SV}}-\gamma_{\mathrm{SL}}}{\gamma_{\mathrm{LV}}}=(1+K) \cos \theta_{\mathrm{Y}}$

Since $K$ is positive, the Wenzel angle is always larger than the Young contact angle. The lower limit of defect sizes that must be included in $K$ is still unknown. This can be an issue in the case of fractal roughness, where $K$ tends towards infinity.

Figure 3 presents a sketch of the total interfacial energy curve as a function of the macroscopic contact angle to illustrate wetting hysteresis, and the possible sticking of the triple line in several metastable states. It is inspired by calculations performed for a meniscus on a vertical sawtooth plate [33], in which the total interfacial energy is taken to be the sum of three terms; i.e., the energy of each interface multiplied by its area. Within a certain range of macroscopic contact angles there are local minima which are separated by energy barriers. The absolute minimum of the curve corresponds to the Wenzel contact angle. Macroscopic contact angles smaller than $\theta_{\mathrm{W}}$, corresponding to

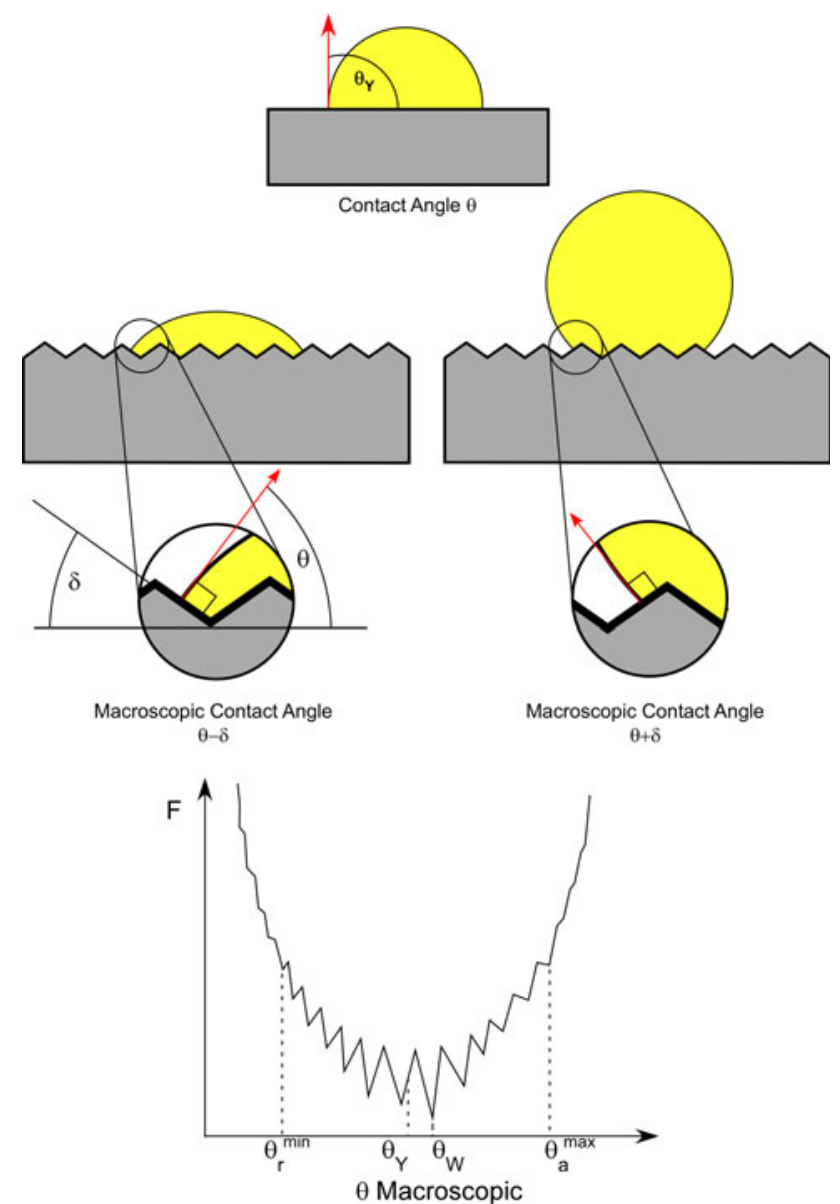

Fig. 3 Wetting on a saw-tooth rough surface. $F=\gamma_{\mathrm{LV}} A_{\mathrm{LV}}+$ $\gamma_{\mathrm{SV}} A_{\mathrm{SV}}+\gamma_{\mathrm{SL}} A_{\mathrm{SL}}$ and the minimum is at $\theta_{\mathrm{W}}$ : the first minimum on the left of the minimum is at $\theta_{\mathrm{Y}}-\delta$ and the last one, on the right is at $\theta_{\mathrm{Y}}+\delta$. The diagram can also be used for the case of heterogeneous surfaces. Then, the Wenzel angle becomes the Cassie angle, but the minimum receding angle (maximum advancing angle) becomes the Cassie angle if the Young contact angle of the chemical defects is higher (lower) than the one on the clean surface

local minima, can be reached by receding the triple line, and conversely, angles larger than $\theta_{\mathrm{W}}$ can be reached by advancing the triple line. The smallest macroscopic contact angle corresponding to a minimum is the minimum receding contact angle $\left(\theta_{\mathrm{Y}}-\delta\right)$. Conversely, the largest macroscopic advancing contact angle is $\left(\theta_{\mathrm{Y}}+\delta\right)$. Their difference defines the width of the wetting hysteresis.

\section{Wetting on chemically heterogeneous surfaces}

A similar type of equilibrium macroscopic contact angle can be defined for a solid with a randomly heterogeneous surface. Consider a surface consisting of two different solids, 1 and 2, with contact angles $\theta_{\mathrm{Y} 1}$ and $\theta_{\mathrm{Y} 2}$ and area fractions $f$ and $1-f$, respectively. Unlike the case of roughness, we only consider one type of defect (of solid 2) on which the contact angle is either smaller or larger than that of 
the clean surface (solid 1). Then, the Cassie equilibrium contact angle, $\theta_{C}$, is given by the following relation [34]:

$\cos \theta_{\mathrm{C}}=f \cos \theta_{\mathrm{Y} 1}+(1-f) \cos \theta_{\mathrm{Y} 2}$

As in the case of rough surfaces, wetting hysteresis also occurs on chemically heterogeneous surfaces. However, in the case of a binary flat surface, the wetting hysteresis does not range across the Cassie contact angle, but rather shifts either between the Cassie angle and a higher contact angle or between a lower contact angle and the Cassie angle. This is because better wetted defects do not cause the advancing triple line to stick and thus do not affect the apparent contact angle, whereas they do cause the receding triple line to stick, thereby inducing smaller contact angles, and vice versa [35].

Other comments on wetting on heterogeneous surfaces

A liquid drop with a triple line that advances or recedes on a surface with disconnected holes, into which the liquid cannot penetrate (hygrophobic wetting [26]), behaves as if it was on a binary surface with one phase having a $180^{\circ}$ contact angle. In that case, the Cassie contact angle is related to the surface fraction of holes through Eq. (6), and the triple line can only stick upon advancing. Consequently, on this kind of surface, the wetting hysteresis always ranges between the Cassie contact angle and higher contact angles [36]. For a very high surface fraction of holes the wetting becomes "superhygrophobic". Both the advancing and Cassie contact angles approach $180^{\circ}$, and wetting hysteresis disappears. This is the origin of the "lotus effect".

When sessile drop measurements are performed, the macroscopic contact angle must be extracted from the overall shape of the drop truncated by the substrate plane. The Wenzel and Cassie contact angles are difficult to measure because the respective absolute minima of the total interfacial energy are surrounded by the highest energy barriers, as shown in the sketch of Fig. 3 [33]. Thus, the measured macroscopic contact angles rarely correspond to $\theta_{\mathrm{Y}}, \theta_{\mathrm{W}}$, or $\theta_{\mathrm{C}}$, but rather to some arbitrary angle somewhere within the range of wetting hysteresis. The values measured for the macroscopic contact angles depend strongly on the location of the triple line, which itself depends on local pinning. The location of the triple line is related to the way in which the liquid drop is formed on the substrate. As an example, Fig. 4 shows the strong effect of micron-sized heterogeneities on the shape of the triple line of a solidified tin droplet; it is pinned on silicon squares, that are better wetted than the silica matrix surface, which produces the wandering of the triple line.

Many other phenomena, such as anisotropic wetting/ spreading of the drop and its motion, can take place on patterned substrates when the size of the surface pattern is

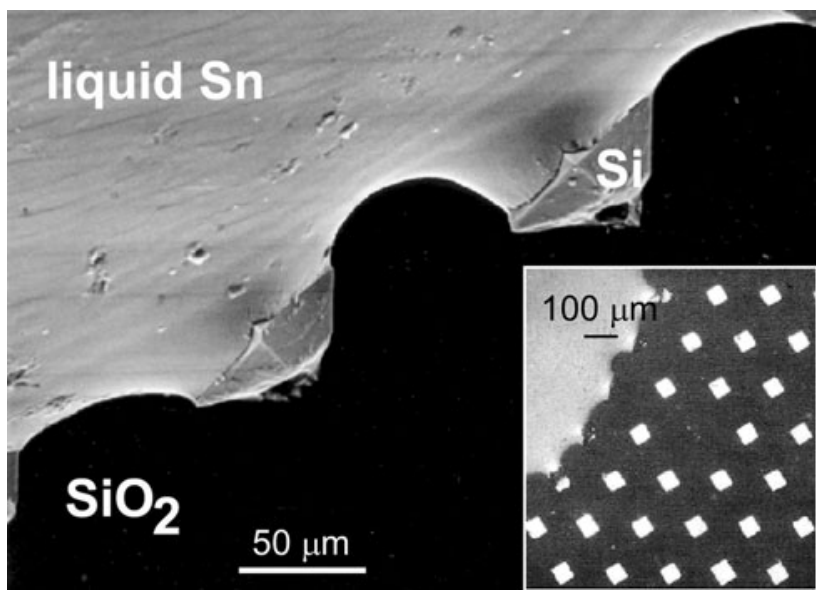

Fig. 4 Secondary electron micrograph of the triple line of a solidified droplet of tin attached to silicon squares organized on a silica surface. The edge of the silicon squares is $50 \mu \mathrm{m}$ ( $3 \mathrm{D}$ triple line). The inset shows a lower magnification micrograph where the silicon squares are white, the silica surface is dark, and the edge of the drop with its wandering triple line is light gray

of the order of the drop size [37]. Control of surface features also allows control of drop and triple line shapes [38]. The literature on these topics is enormous, especially in the field of room-temperature wetting.

In this section, phenomena that can lead to metastable wetting states, i.e., triple line positions, have been described. It should be emphasized that the measured contact angle will depend on the metastable state in which the triple line is trapped. Different states can be reached depending on the kinetics of the triple line, which have not been addressed specifically in this paper. However, the reader should be warned that the correct interpretation of a measured contact angle requires a thorough characterization of both the drop and the solid substrate on which the wetting experiment is performed, and on the manner in which the triple line has reached its location on the substrate.

\section{Contact angles near triple lines: interaction between interfaces}

In the vicinity of the triple line, the distance between interfaces becomes very small, which can lead to interactions between them. These interactions occur because of the finite thickness of interfaces (as described in later sections), and can in turn produce local distortions of the liquid surface, which may be displaced either towards or away from the solid/liquid or the solid/vapor interfaces, as depicted in Fig. 5. These distortions can produce excess energies of the order of $10^{-9} \mathrm{~J} / \mathrm{m}$ if assigned to the triple line (see for example [39, 40]). As a result of these distortions, a contact angle defined by the equilibrium of the macroscopic interfacial energies should never be measured 


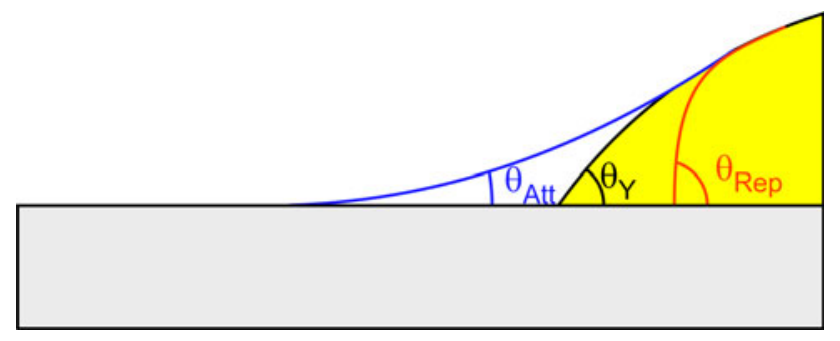

Fig. 5 Sketch of the deviation of the liquid surface at the triple line of a sessile drop under the influence of attractive interactions between two surfaces on the apparent $\left(\theta_{\text {Att }}\right)$ contact angle, versus the influence of repulsive surface interactions leading to an apparent contact angle $\left(\theta_{\mathrm{Rep}}\right)$ approaching $90^{\circ}$

too close to the triple line. As mentioned before, the best approach for measuring a macroscopic contact angle is to fit the shape of the liquid surface with a relevant function and truncate that shape by the substrate plane.

\section{Wetting on unconstrained isotropic substrates}

Wetting on a deformable substrate such as a liquid, shown schematically in Fig. 6 is characterized by a dihedral angle, $\phi$, within the lenticular cap of the partially wetting phase. In the case of a three-phase, liquid 1 (L1)-liquid 2 (L2)vapor $(\mathrm{V})$ system in which all surface energies are isotropic, the dihedral angle is related to the interfacial energies by the Neumann relationship:

$\frac{\gamma_{\mathrm{L} 1 \mathrm{~V}}}{\sin \beta}=\frac{\gamma_{\mathrm{L} 1 \mathrm{~L} 2}}{\sin \alpha}=\frac{\gamma_{\mathrm{L} 2 \mathrm{~V}}}{\sin \phi}$

The equilibrium shape of the confined phase corresponds to the minimum of the total interfacial energy which is the sum of the energy of each interface multiplied by its area. In order for the total interfacial energy to be minimized, the interfaces between L1 and L2 and the L1 surface, which confine the L1 drop, adopt the shape of spherical caps. The schematic in Fig. 6 is valid in the absence of buoyancy, and for isotropic interfaces; with

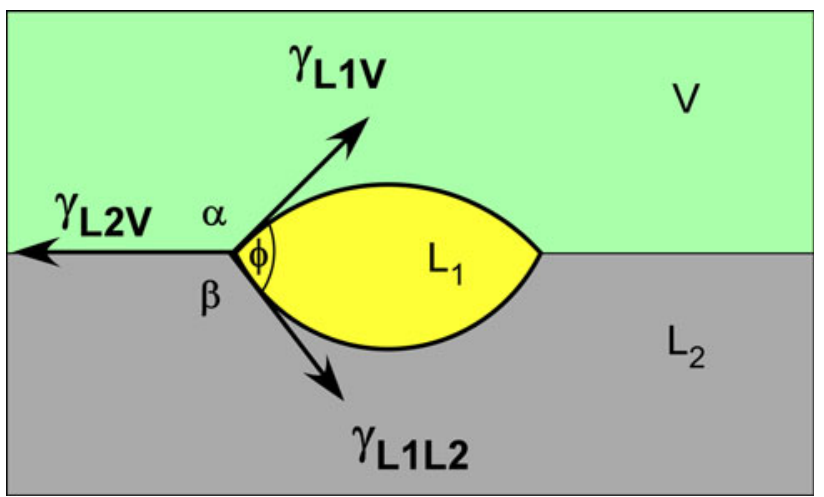

Fig. 6 Wetting on a deformable surface and the resulting lenticularshaped drop with dihedral angle $\phi$ buoyancy the $\mathrm{L} 2 \mathrm{~V}$ interface will be curved. When the $\mathrm{L} 2 \mathrm{~V}$ interface is flat, the values of surface and interface energyweighted curvatures on the two sides of the $\mathrm{L} 1$ lens must be equal: $\gamma_{\mathrm{L} 1 \mathrm{~V}} / R_{\mathrm{L} 1 \mathrm{~V}}=\gamma_{\mathrm{L} 1 \mathrm{~L} 2} / R_{\mathrm{L} 1 \mathrm{~L} 2}$ For the case illustrated in Fig. 6, it should be emphasized that the "apparent contact angle" above the level of the flat surface of the substrate is not related to the interfacial energies by Young's equation (1).

An isotropic particle embedded in an internal interface will also adopt a lenticular shape, and the wetting may be characterized by the dihedral angle, $\phi$, of the particle at the triple junction. A dihedral angle may also be used to describe the equilibrium angle at the groove that forms at the intersection of a grain boundary (or two-phase boundary) with another interface (see Fig. 7). The dihedral angle shown in Fig. 7a relates the energies of the interfaces on each side of the groove, $\gamma_{1}$ and $\gamma_{2}$, to the boundary energy, $\gamma_{12}$, as expressed by the Neumann equation (Eq. 7). This condition may also be expressed as a vector equilibrium resolved in the horizontal and vertical directions:

$$
\begin{aligned}
& \gamma_{1} \cos \phi_{1}+\gamma_{2} \cos \phi_{2}=\gamma_{12} \\
& \gamma_{1} \sin \phi_{1}=\gamma_{2} \sin \phi_{2} \\
& \phi_{1}+\phi_{2}=\phi
\end{aligned}
$$

It is more usual to find the dihedral angle at a grain boundary defined by Eq. (9), with the restriction of a symmetrically shaped groove (where $\gamma_{i 1}=\gamma_{i 2}=\gamma_{i}$ ) (see Fig. 7b, c).

$\cos \frac{\phi}{2}=\frac{\gamma_{12}}{2 \gamma_{i}}$

Note that the shapes of the surfaces around the groove are kinetic shapes [41] but the angle at the groove is an equilibrium angle.

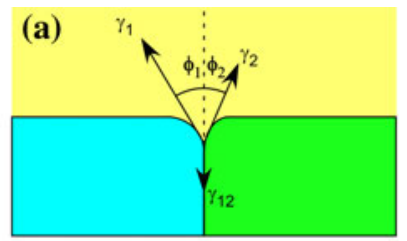

Symmetrical Grain Boundary Grooves

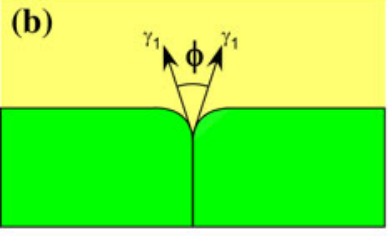

Bulk Diffusion

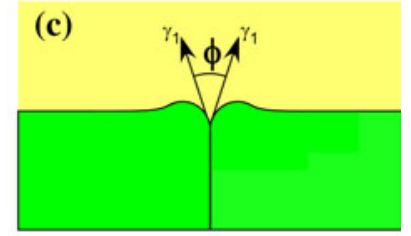

Interface Diffusion
Fig. 7 Wetting and grooves at internal interfaces: a general case, b and c classical sketches for a symmetrical grain boundary groove equilibrated under two different mechanisms of solid diffusion $[41,79]$ 


\section{Wetting and anisotropic interfaces}

The assumption of interfacial energy isotropy is only valid for the interfaces of fluids and amorphous solids, whereas interfaces that involve crystalline solids (or liquid crystals) are anisotropic. Interface anisotropy issues are addressed in two subsections. The first one will describe the wetting of a crystal on a flat substrate, and the second one, the wetting of an unconstrained anisotropic substrate.

\section{Equilibrium crystal shape}

First, it is useful to introduce the concept of the ECS, or Wulff shape, of a crystal equilibrated in a vapor phase (see Fig. 8). The ECS can be obtained from a polar plot of the orientation dependence of surface energy $(\gamma / \hat{n})$, the socalled $\gamma$-plot, by means of the Wulff construction [42] (where $\hat{n}$ is a unit vector normal to the surface). The ECS is convex and conveniently centered on a point referred to as the Wulff point. It may display facets (atomically flat surfaces of given orientations) and curved surfaces (atomically rough orientations). Facets occur at orientations that correspond to cusps on the $\gamma$-plot. The deeper the cusp, the lower the surface energy of this orientation, and the larger the corresponding facet on the ECS. All the orientations which exist on the ECS are stable. All orientations will be stable for the case of an ECS with facets, when facets and curved parts connect tangentially (see Fig. 8b). If a discontinuous (sharp) connection appears on the ECS, some orientations will be missing and thus unstable (see Fig. 8c) [43]. For example, for a face-centered cubic (fcc) crystal with an ECS in the shape of a cubo-octahedron, consisting of the $\{111\}$ and $\{100\}$ facets, these will be the only two stable orientations. The unstable orientations have a virtual energy, which cannot be measured experimentally. Such orientations decompose into micro-facets of the adjacent stable orientations present on the ECS (Fig. 8c). Their effective energy can be extracted from the ECS as suggested by Herring [44]:

$\gamma=\frac{1}{a_{0}} \sum_{i} \gamma_{i} a_{i}$

where $a_{0}$ is the area of the unstable plane, $i$ represents the stable facet types, and $a_{i}$ and $\gamma_{i}$ are the $i$ th facet area and surface energy, respectively (see Fig. 8d).

\section{Solid-state wetting}

Until this point we have dealt primarily with the concepts involved for wetting of a liquid in contact with a solid. These issues are important for a fundamental understanding of solid-liquid interfaces, and critical for engineering methods which depend on solid-liquid interfaces, such as

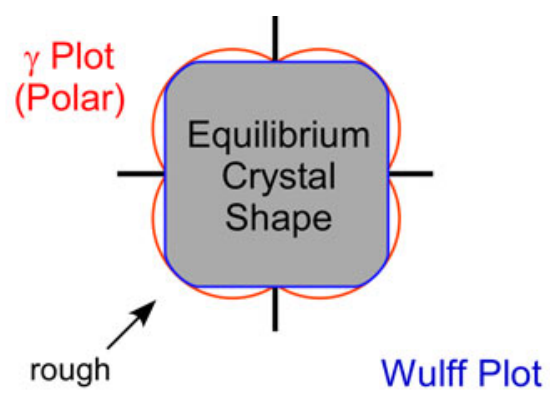

All Orientations

(b)

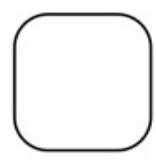

Missing

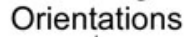

(c)

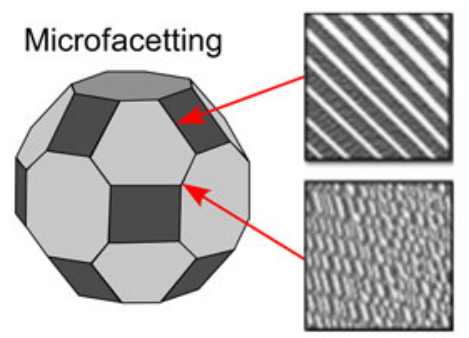

(d)

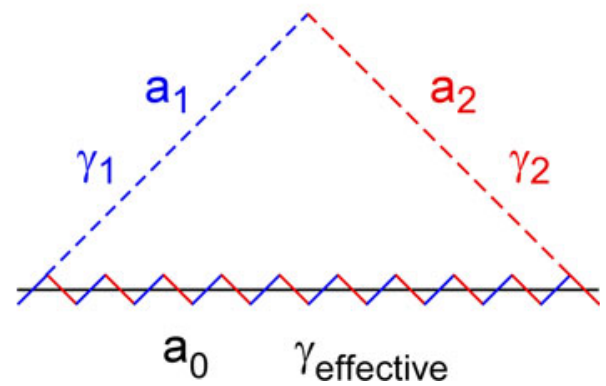

Fig. 8 Equilibrium shapes and faceting: a 2D $\gamma$-plot and equilibrium shape of a crystal; b two 2D equilibrium shapes, the left one with all the orientations and the right one with missing orientations where the shape has singularities; c 3D equilibrium shape of an fcc crystal with only three types of stable orientations $(\{111\},\{100\}$, and $\{110\})$; d break-up of an unstable facet into two facets with energy $\gamma_{1}$ and $\gamma_{2}$. On the right of c, two AFM micrographs show microfacetting of unstable orientations between two stable facets or three stable facets

solidification, soldering, and brazing. However, solid-solid interfaces are equally important for numerous technological applications as well as for fundamental studies. One fundamental goal of solid-state wetting analysis is to extract the interfacial energy between two solids. This important fundamental parameter can be used in the Young-Dupré equation (Eq. 4) to obtain the thermodynamic work of 
adhesion for solid-solid interfaces, which defines the lower limit of the fracture energy of an interface (ignoring dissipative processes) [45].

Why contact angles of solid crystals on a substrate should not be used?

Given the approach of Young described previously, the natural tendency of the experimentalist is to simply measure the apparent contact angle of an equilibrated crystal on a flat solid substrate, as is done in the sessile drop experiment. Unfortunately this approach is overly simplistic, and ignores the influence of the anisotropic crystal shape on the apparent contact angle. This problem is demonstrated via the simple schematic in Fig. 9a, which shows a crystal equilibrated in contact with a flat and rigid substrate, and the apparent contact angle $\theta$. As will be discussed later in section "Microscopic scale and adsorption", adsorption at interfaces can modify the interfacial energy. Let us suppose a hypothetical case where an additional component is added to the system of Fig. 9a, such that it adsorbs only to the interface between the substrate and the crystal (i.e., not to the free surface of the particle or the substrate) and decreases its energy. As a result, the total surface/interface areas are optimized in order to minimize the total surface/interfacial energies, and the relative interface area in Fig. $9 \mathrm{~b}$ is increased versus Fig. 9a, for a crystal of the same volume. However, due to the equilibrium shape of the crystal, the facets have not changed and neither has the apparent contact angle. Thus a measure of solid-state wetting via apparent contact angles is obviously an erroneous approach.

\section{Winterbottom analysis}

An approach to deal with experimental measurement of solidsolid interfacial energies was developed by Winterbottom [46]. This approach is based on a geometrical analysis of the Wulff shape of a crystal equilibrated on a flat solid substrate of a dissimilar material, under conditions of constant temperature, volume and chemical potentials. The Winterbottom construction is described in detail in "Panel 2".

\section{Panel 2: Winterbottom analysis}

The Winterbottom construction generates the equilibrium shape of a crystal - with fixed volume - that is attached to a flat substrate. The traditional description of the Winterbottom construction is that the center of Wulff shape is displaced in the direction of the substrate by a distance given by the difference of the crystal-substrate

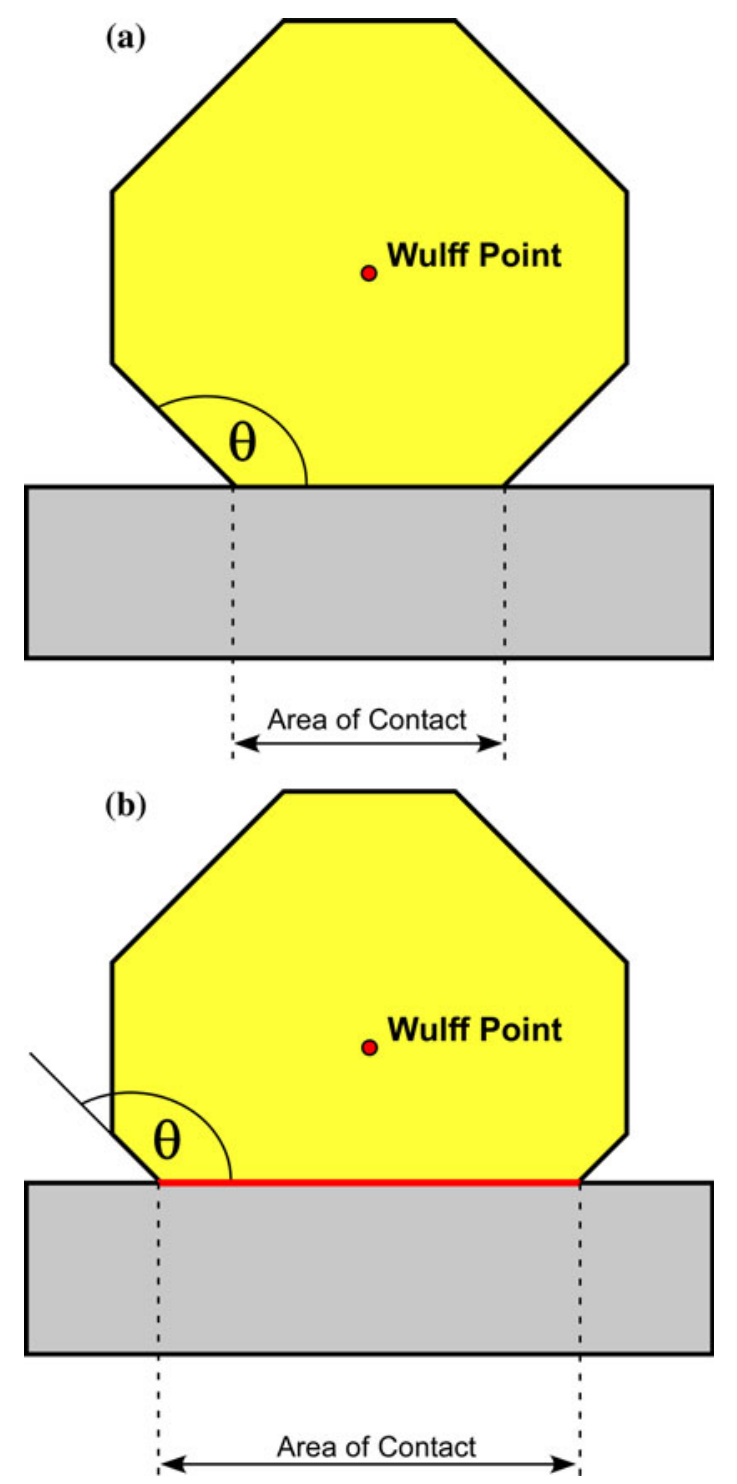

Fig. 9 Schematic drawing of a single crystal equilibrated in contact with a flat solid substrate. The apparent contact angle $\theta$ in a remains the same in $\mathbf{b}$ where the interface energy has been reduced due to segregation (indicated by the red line at the interface) (Color figure online)

interfacial energy minus the substrate-vapor surface energy. However, the derivation of this construction is questionable [46], and cannot apply to the general case where the Wulff shape does not have a unique center, such as crystal for which the point group lacks an inversion center [47].

Figure P2-1 justifies and generalizes the Winterbottom construction. The Wulff construction of the isolated crystal in equilibrium with a vapor is illustrated in Fig. P2-1, for a $\gamma$-plot in which $\gamma_{\mathrm{cv}}(\hat{n}) \hat{n}$ is drawn in red. The thin black lines are drawn for discrete values of interface orientation and are perpendicular to the gamma vectors for each orientation. Suppose that the substrate is isotropic and constrained to be flat. To this $\gamma$-plot, an 


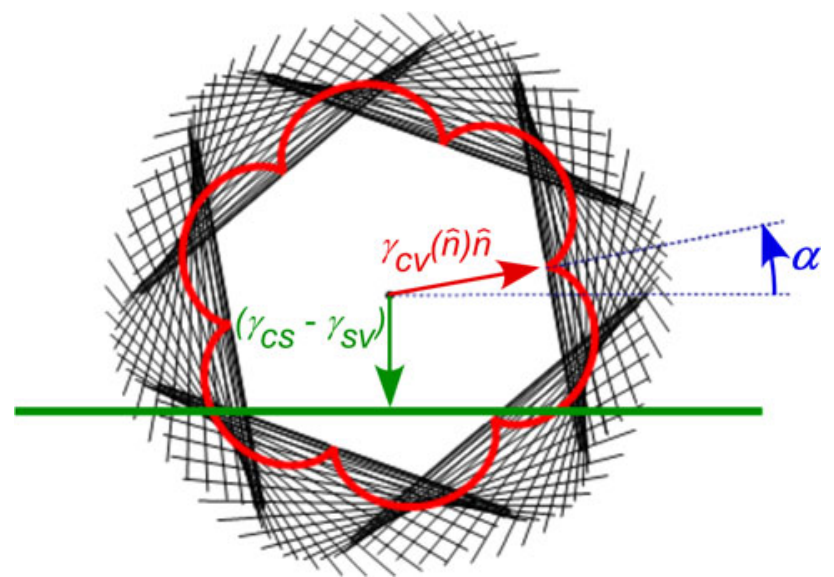

Fig. P2-1 Winterbottom construction on a flat substrate

additional surface energy must be superposed that represents the interfacial energy due a crystal/substrate interface oriented $\gamma_{c s}(\alpha=\pi / 2)(\hat{n}=(0,1))$ minus the surface energy of the vapor/substrate interface oriented in the opposite direction: $\gamma_{\mathrm{sv}}(\alpha=-\pi / 2)(\hat{n}=(0,-1))$. In the case of isotropic interfacial energy for the crystal/ substrate and substrate/vapor interfaces this reduces to a $\gamma$-interfacial vector with magnitude $\gamma_{c s}-\gamma_{v s}$. This interfacial vector is illustrated in green in Fig. P2-1. Performing the Wulff construction on superposed surface energies yields the equilibrium shape of the crystal attached to the substrate. When $\gamma_{\mathrm{cs}}-\gamma_{\mathrm{vs}}-\gamma_{\mathrm{cv}}>0$, i.e., the interfacial vector lies exterior to the crystal/vapor Wulff shape, the crystal/vapor Wulff shape separates from the substrate, and the vapor phase intrudes between the two solids. The transition to complete wetting occurs when $\gamma_{\mathrm{cv}}+\gamma_{\mathrm{cs}}-$ $\gamma_{\mathrm{vs}}<0$ i.e., when the "interfacial vector" touches the top of the crystal/vapor Wulff shape. In this state, the surface will be composed of any facets in the Wulff shape that are adjacent to the top point of the crystal/vapor Wulff shape; the only constraint is that the combinations of these facets produce an average orientation that is normal to the interface. However, this transition to complete wetting is more subtle: consider the range of interfacial vectors which give the same "shape" (e.g., in Fig. P2-1, those with magnitude greater than the top right point on the hexagon and less than the highest point on the hexagon). These will all produce the same morphology. However, at complete wetting, a continuous facetted film that entirely covers the substrate is formed, with microfacets of the same orientations as those present before complete wetting prevails. Thus, the transition to complete wetting can be directly observed.

Before generalizing this construction below, it is useful to point out a property of the Wulff construction. The crystal/vapor Wulff shape (illustrated in Fig. P2-1 as a hexagon) has an infinite set of $\gamma$-plots that produce this exact same shape. That is, any other $\gamma$-plot which is exterior to the one illustrated above, but has cusps which coincide with the particular $\gamma$-plot, will produce the same Wulff shape. When the border of the $\gamma$-plot is composed of circles (spheres in 3D) they are tangent to the origin. These are the circles from the Frank construction which is equivalent to the Wulff construction [48]. This minimal set of $\gamma$ corresponds to a convexification in polar (spherical in 3D) coordinates [49]. The following construction depends on the following property: each point on the convex set (i.e., circles) has a surface energy that is the same as the linear combination of neighboring facets which produce the same average orientation represented by that orientation on the set (i.e., the point at the circle at a particular angle from the origin).

It is straightforward to generalize this method of utilizing the Wulff construction to produce a Winterbottom shape for the case where each surface and the interface are anisotropic. This construction is illustrated in Fig. P2-2. In this case, the vapor/substrate surface energy is obtained from the substrate's average macroscopic normal. As illustrated in the left-hand portion of Fig. P2-2, the vertical normal is composed of two facets which do not have a vertical orientation, but combined in such a way that the average normal is the same as the substrate's. The morphology of the uncovered vapor/ substrate is illustrated as a jagged surface; the effective surface energy of this morphology is independent of the scale of the facet lengths (these are varifolds); therefore an infinitesimal amount of mass transport will give rise to such microfaceting. Because the characteristic length scale can be arbitrarily small, the development of such morphologies produces finite reductions in effective surface energy and takes place in arbitrarily short times, even if diffusion is slow.

A crystal/substrate interface energy is produced in an analogous fashion but with its normal obtained from the downward vertical direction (illustrated in blue in the right-hand portion of Fig. P2-2). Similarly, the morphology of the crystal/substrate interface derives from the corresponding orientation of the crystal/substrate Wulff shape. The interfacial vector is now represented by the sum of the two (oppositely oriented) vapor/ substrate and crystal/substrate effective interfacial energy. As above, the Wulff construction predicts that the distance (in units of $\gamma$ ) of the Wulff shape to the average substrate position is given by the interfacial vector. The construction above is the generalization to the lens construction on a flat isotropic substrate; in the generalized construction the flat interfaces are replaced with those that have zero-weighted mean curvature 
Fig. P2-2 Winterbottom construction in the case all surfaces and the interface are anisotropic (Color figure online)
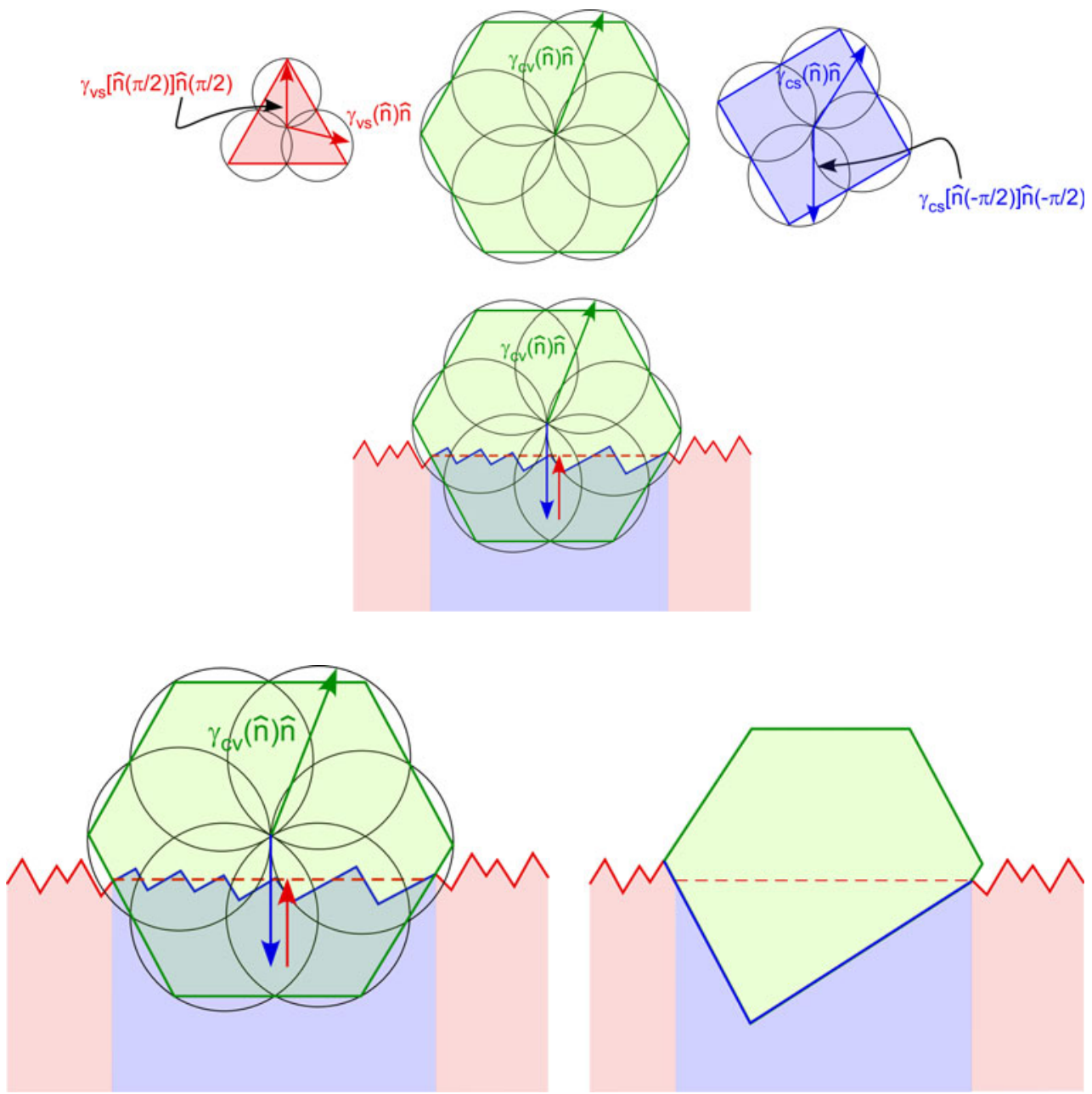

Fig. P2-3 Winterbottom construction in the case all surfaces and the interface are anisotropic and the interface is "deformable"
[50]. The generalization to the case where the substrate is deformable is treated below.

The construction for a deformable crystal/substrate interface is given in Fig. P2-3 as the transition from a jagged interface (with the same average normal as the substrate's) (on the left) to the deformed equilibrium interface composed of two consecutive facets of the Wulff shape (on the right) with the same crystal volume; this is a generalization of the double lens construction that balances both the vertical and horizontal components of the surface tensions at the triple line, and produces two circular (or spherical) caps, such that the product of the surface tension times the curvature of the cap is the same as the lens' opposite interfaces. In the general faceted case, the only requirement is that the weighted mean curvature be the same on each interface of the crystal. The weighted mean curvature is the rate of interfacial energy increase with respect to addition of volume (heuristically, $\partial(\gamma A) / \partial V)$. The equivalence of weighted mean curvature guarantees that there is no change in total energy if material from one facet is transported to another, which is a necessary condition for equilibrium.

The interfacial energy can be determined by measuring two characteristic lengths in the Wulff shape of the crystal truncated by the substrate: the distances from the Wulff point of the crystal to the interface with the substrate $\left(R_{1}\right)$, and from the Wulff point to the uppermost facet ${ }^{4}$ of the crystal $\left(R_{2}\right)$, as shown schematically in Fig. 10, which provides a basis for this method.

For the sake of simplicity, we have considered the case of a centro-symmetric crystal with a facet parallel to the substrate. When the effective contact angle is larger than $90^{\circ}$ (see Fig. 10a), the Wulff point is above the interface with the solid substrate. The measured values of $R_{1}$ and $R_{2}$ can then be used to determine the interfacial energy according to:

\footnotetext{
${ }^{4}$ Actually, any facet of the Wulff shape of the crystal can be chosen for a measure of $R_{2}$ under the condition that it is not truncated by the substrate.
} 


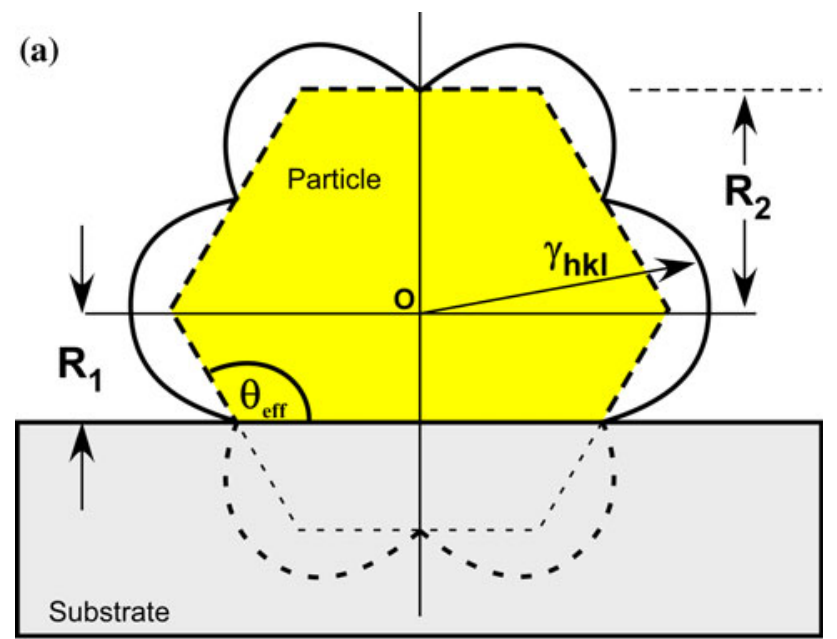

(b)

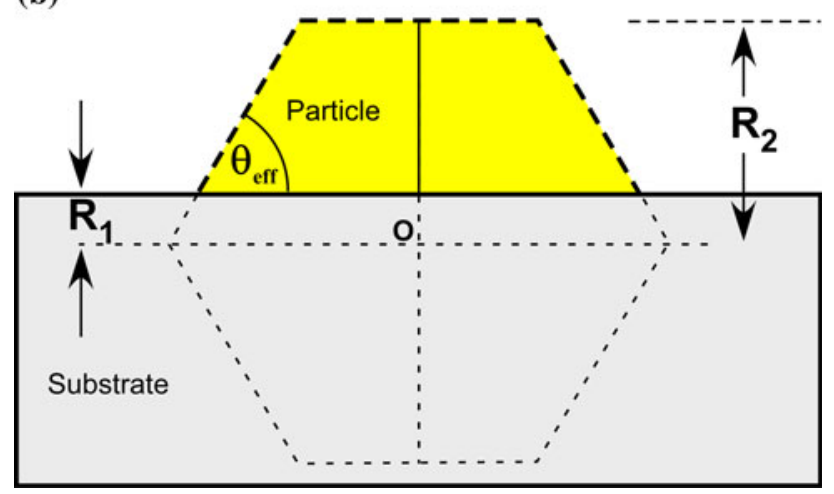

Fig. 10 Schematic drawing of the Winterbottom analysis for particles equilibrated on a substrate, having different effective contact angles: a $\theta<90^{\circ}$ and $\mathbf{b} \theta>90^{\circ}$. The dashed polyhedron indicates the resulting equilibrium Wulff shape and its center $\mathrm{O}$ (the Wulff point). $R_{1}$ and $R_{2}$ are the distances from the Wulff point to the interface with the substrate and to the uppermost particle facet, respectively

$\frac{R_{1}}{R_{2}}=\frac{\gamma_{\mathrm{SC}}-\gamma_{\mathrm{SV}}}{\gamma_{\mathrm{CV}}}$

where $\gamma_{\mathbf{S C}}$ is the substrate-crystal interfacial energy, $\gamma_{\mathbf{S V}}$ is the surface energy of the substrate, and $\gamma_{\mathrm{CV}}$ is the surface energy of the uppermost crystal facet $[46,51]$. Note that this equation can also be used for a particle with an isotropic surface (in the shape of a spherical segment (like the sessile drop of Fig. 1)) where the ratio of radii $R_{1} / R_{2}$ can be replaced by the cosine of the Young contact angle. Thus, $\cos ^{-1}\left(R_{1} / R_{2}\right)$ may be viewed as an effective contact angle for faceted particles on a substrate.

Although Winterbottom's analysis provides a relatively simple methodology for the measurement of $\gamma_{\mathrm{SC}}$, past experimental limitations prevented widespread applications. Initially, the Winterbottom analysis was used only in isolated studies [51-54]. The major limitation arises from the demand that the examined system consist of a single crystal particle thermodynamically equilibrated and having a flat interface which is co-planar with the substrate surface [55]. The requirement for equilibration (in a reasonable time frame), in addition to the absence of grain boundaries, limits the particle size. This poses a challenge regarding the characterization techniques for high accuracy morphological analysis. In addition, the macroscopic degrees of freedom defining the relative orientation of the crystal with the substrate should be measured, and thus the orientation of both the crystal and the substrate should be determined. With the introduction of dual-beam focused ion beam (FIB) systems, it is now possible to accurately prepare cross section samples from the center of small crystals equilibrated on substrates [56, 57], and make accurate measurements of solid-solid interfacial energy and orientation relationships between the crystal and the substrate [58]. Furthermore, if the cross section transmission electron microscopy (TEM) sample is thin enough [59, 60], more advanced TEM techniques can be used to determine the atomistic structure and chemistry of the interface for the same sample [61-63]. The size of the crystals must not be too small, since clusters in the nanometer length scale may exhibit variance of equilibrium shapes depending on the particle size and hetero-epitaxial related interfacial stress [64-67].

The use of Eq. (11) requires knowledge of the absolute values of the relevant surface energies, if an absolute value of the interface energy is the goal. Adsorption may occur to the surfaces, resulting in changes in their chemical composition and lead to a decrease in the surface energies, which can be estimated if the chemical potentials are known (see section "Jumps in adsorption do not mean jumps in surface energy"). While the surface composition can be determined by analytical TEM techniques or atom probe tomography, measurement of the relevant surface energies is a major obstacle, and systematic experimental approaches are needed.

Another obstacle lies in the application of this approach to systems in which the effective contact angle is smaller than $90^{\circ}$ (illustrated in Fig. 10b). In this case, less than half of the complete Wulff shape (of an isolated crystal) is visible, making determination of the Wulff point less accurate (even impossible). Hansen et al. [54] encountered this problem when applying Winterbottom's analysis to a $\mathrm{Pd}-\mathrm{Al}_{2} \mathrm{O}_{3}$ interface. Their solution was to superimpose the calculated Wulff shape of Pd on the particle morphology in order to measure $R_{1}$ and $R_{2}$. Assuming this approach is valid, which necessarily assumes no changes in the Wulff shape of the experimentally characterized particle compared to the simulated Wulff shape, then: 
$\frac{R_{1}}{R_{2}}=-\left(\frac{\gamma_{\mathrm{SC}}-\gamma_{\mathrm{SV}}}{\gamma_{\mathrm{CV}}}\right)$

Wetting on unconstrained anisotropic substrates

\section{Effects of interfacial torques on wetting equilibrium}

Wetting on a rigid flat solid substrate by means of the classical Young equation has been described above. That configuration is simple, but it only describes equilibrium under the constraint that the substrate remains flat. At high temperatures, where mass transport processes can play a role, the substrate interface will change shape so as to allow the triple junction of three isotropic interfaces to satisfy the Neumann equation (Eq. 7).

The Neumann equation corresponds to the isotropic limit of the more general Herring equation of interfacial equilibrium, which takes into account the anisotropy of interfacial energies by including "torques" [44], defined as the derivatives of interfacial energy with respect to orientation. The complete equilibrium of three interfaces at a triple junction has been given by Herring [44] as:

$\sum_{i=1}^{3}\left(\gamma_{i} \vec{t}_{i}+\frac{\partial \gamma_{i}}{\partial \vec{t}_{i}}\right)=0$

where $\gamma_{i}$ are the three interfacial energies, $\vec{t}_{i}$ is the vector in the plane of the $i$ th interface, normal to the triple line and pointing away from it; and the torque, $\partial \gamma_{i} / \partial \vec{t}_{i}$, is a vector perpendicular to $\vec{t}_{i}$ and to the triple line (see Fig. 11) [68]. If the orientation of one of the interfaces corresponds to a cusp in the $\gamma$ plot, then $\partial \gamma_{i} / \partial \vec{t}_{i}$ is indeterminate, but equilibrium can still prevail as long as $\partial \gamma_{i} / \partial \vec{t}_{i}$ adopts a value that lies between the two limiting slopes on the sides of the cusp (Fig. 11a). Thus, if in the case of a classical sessile drop configuration, such as Fig. 1, the torques of the solid/ fluid and of the substrate/vapor interfaces correspond to cusps, but lie between limits that can balance the pull of the liquid surface tension perpendicular to the substrate (i.e.,
$\left.\gamma_{\mathrm{LV}} \sin \theta_{\mathrm{Y}}\right)$ then both of these interfaces will remain flat and coplanar. This is a case where the Young equation applies at high temperature.

Deviations from Young and Winterbottom conditions: double Winterbottom construction and ridging

The Young equation for drops is valid, as long as the interface is flat, and co-planar with the surface of the substrate. However, this condition will only be met when the torques of the crystallographic plane parallel to the surface of the substrate and its interface with the crystal are significant, as explained above. If this condition is not met, then an isotropic drop will adopt an equilibrium shape such as that described in Fig. 6. In the case of an anisotropic crystal on a crystalline substrate, the corresponding equilibrium shape will be as shown in the right panel of Fig. P2-3, where both the crystal surface and the crystalsubstrate interface may be facetted. This amounts to applying the Winterbottom analysis to both the crystal surface and the crystal-substrate interface [69, 70], as has been described in "Panel 2", and which is generally referred to as the double Winterbottom construction.

This effect has been specifically characterized for particles at grain boundaries [71, 72], and numerically modeled for particles equilibrated on a solid surface or at grain boundaries [73, 74]. More recently, Zucker et al. [75] have developed a simulation tool which can be used to both model and extract relative surface and interface energies for such complicated morphological systems. One of the expected critical outcomes of these types of simulations will be to determine whether the interface deviates from planarity, at length scales relative to the size of the particle (see the examples described in Fig. 12).

If the torques of the solid/drop or solid/crystal and the substrate/vapor interfaces are insufficient to balance the vertical pull of the surface energy of the confined phase
Fig. 11 a Schematic crosssectional view of a triple junction, where $n_{i}$ are unit vectors in the direction $\partial \gamma_{i} / \partial \vec{t}_{i}$ (after Saylor and Rohrer [68]). b Grains with isotropic surface energies result in a rough triple junction, while anisotropic surface energies result in c faceted surface planes (a)

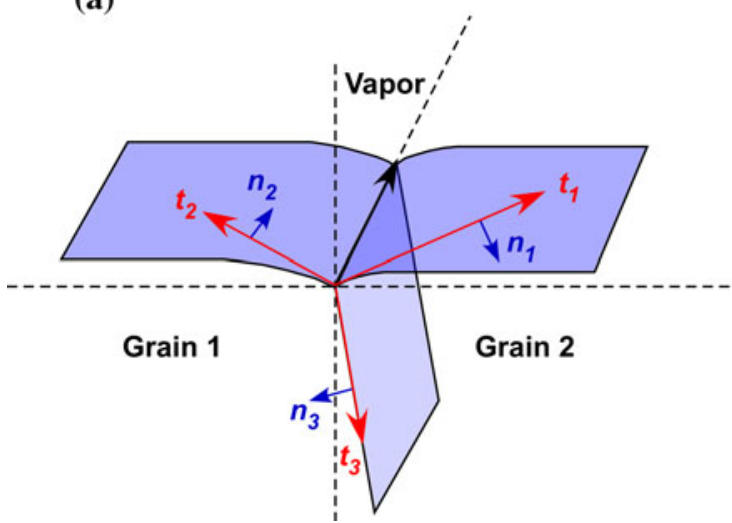

(b)

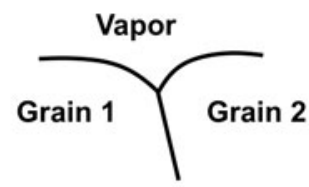

(c)

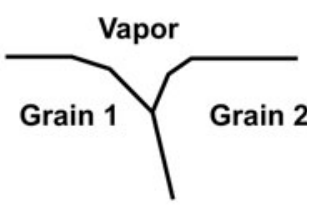


Fig. 12 Three particle shapes calculated for the abutment of a a cube and a sphere attached to a (100)-type boundary, $\mathbf{b}$ an octahedron and a sphere attached to a (111)-type boundary, and $\mathbf{c}$ an octahedron and a sphere attached to a (110)type grain boundary. Reprinted with permission from [73]
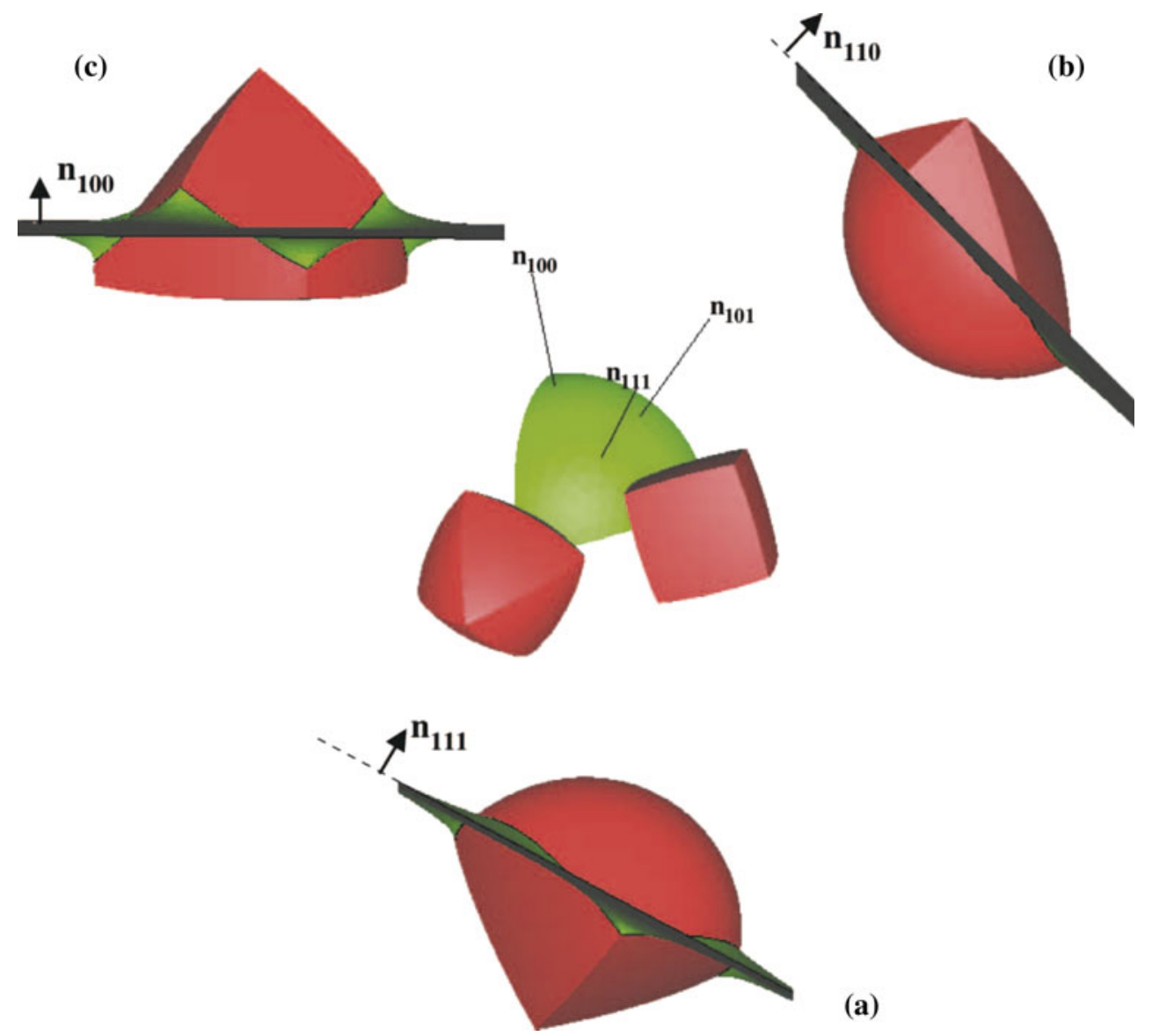

(a)

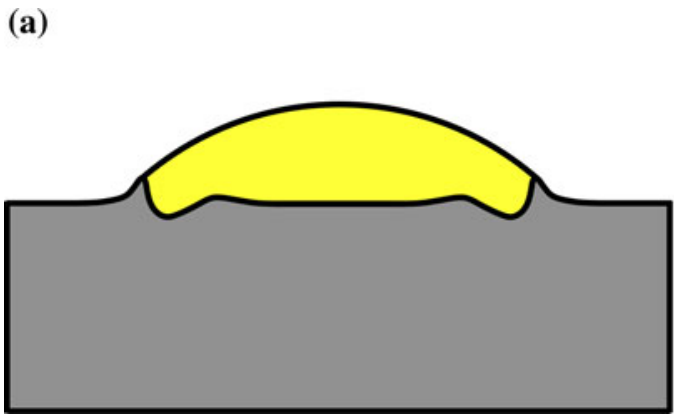

(b)

Fig. 13 a Schematic drawing of ridging at a triple junction due to unbalanced torque terms, and $\mathbf{b}$ a SEM micrograph of a faceted ridge formed at the triple line of an copper drop equilibrated on a sapphire surface (b was reproduced with permission from [78])

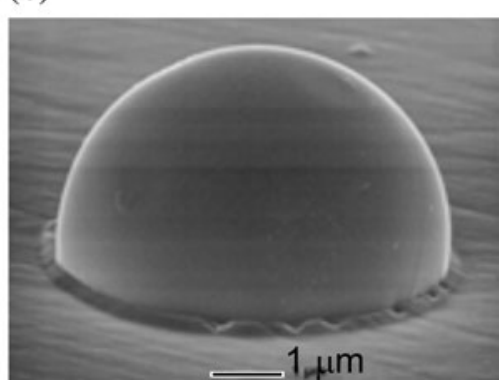

(drop or crystal), and transport of matter near the triple line is sufficiently rapid, then mass transport of the solid will allow the local equilibrium angles required by the Neumann equation (Eq. 7) to develop.

Under these conditions, a transient ridge will form at the triple line [76, 77] of a sessile drop or a sessile crystal, as shown in Fig. 13 for a copper droplet annealed above its melting point on a sapphire substrate [78]. This phenomenon is similar to the well-known phenomenon of grain boundary grooving, which occurs where a grain boundary intercepts a surface or an interface [41, 79] (see Figs. 7, 11). It should be noted that the detailed shape evolution of ridges (or of grooves at a grain boundary) depends on kinetics [41, 79], whereas the angles are always determined by the equilibrium equations, as local interfacial energy equilibrium always prevails.

The relative size of the ridge and the drop radius can have a significant effect on whether the observed macroscopic contact angle follows the Young equation. For relatively small ridges, the departure from Young's equation is small; however, for relatively large ridges the apparent contact angle can be quite different from that expected from Young's equation. Details on the kinetics of ridging are given in [76]. On the other hand, the presence of ridges can be used to determine interfacial energies [80], as in the case of grain boundary grooving.

In spite of its importance, as indicated in the above discussion, information on the orientation dependence of 
interfacial energy is available only for a limited number of pure fcc metals and alloys and for a few simple oxides. As a result, the torque terms in Eq. (13) have often been neglected owing to insufficient data.

\section{Dewetting and spreading}

While the term 'wetting' has been used throughout this text as the description of a thermodynamic state (e.g., reflected by a contact angle), 'wetting' is sometimes incorrectly used to describe the kinetics of spreading of a liquid across a solid. The kinetics of spreading, which is driven by the minimization of surface and interface energy (defined by wetting), is obviously an important technological topic. In order to prevent confusion, we adopt 'spreading' to describe the kinetic changes of surface and interface area, and 'wetting' to describe thermodynamics (equilibrium). The term "dewetting" has also gained general use to describe the break-up (agglomeration) of a thin film in the liquid (or solid state) into drops (or particles), driven by the minimization of surface and interfacial energy in a system where the three coexisting phases are at equilibrium. While the kinetics of dewetting can be studied to reach important conclusions regarding surface transport, the equilibrium state reached by the kinetic process of dewetting offers an alternative approach to study the equilibrium state of solid-liquid and solid-solid interfaces. A recent review by Thompson [81] has been dedicated to this phenomenon. In the following we report on the main points of dewetting relevant to this paper.

Spinoidal dewetting versus nucleation of holes

There are two main proposed mechanisms for dewetting of thin films on solid surfaces: spinodal dewetting (which mostly refers to the Raleigh instability) and nucleation of voids [82]. The mechanism for the Raleigh instability in liquid-state dewetting is a wave fluctuation which grows exponentially with thermal activation, resulting in two opposite surface curvatures and the tendency to minimize surface area by breaking the film up into droplets to reach equilibrium [79]. For solid-state dewetting, spinodal dewetting occurs mostly for thin films, where the amplitude of the fluctuations is large enough to form hills and depressions via surface diffusion. When the depressions reach the substrate, agglomerated particles are formed, or break-up of the film occurs [83]. Recently, faceted film-edges were observed without depressions [84, 85] (due to surface energy anisotropy) and confirmed by a model developed by Klinger et al. [86].

In polycrystalline films, solid-state dewetting takes place either by extension of grain boundary and triple junction grooves [87, 88] from the free surface to the interface, or via nucleation of voids at the interface (see
Fig. 14). Following Srolovitz and Safran [82] and using the criteria of grain boundary grooves extending from the free surface to the interface, a polycrystalline film of thickness, $a$, will rupture if:

$\frac{R}{a} \geq \frac{3 \sin ^{3} \beta}{2-3 \cos \beta+\cos ^{3} \beta}$

where $R$ is the grain size, and $\beta$ is defined in terms the grain boundary $\left(\gamma_{\mathrm{GB}}\right)$ and surface $\left(\gamma_{\mathrm{SV}}\right)$ energies as $\beta=$ $\sin ^{-1}\left(\gamma_{\mathrm{GB}} / \gamma_{\mathrm{SV}}\right)[82,83,89,90]$. This model ignores the influence of the film-substrate interfacial energy, and if the interfacial energy is relatively high and a mechanism exists for nucleation of voids at the interface, then voids will first form at the interface, and grow towards the surface [91, 92]. The details of the mechanism for void nucleation at the interface are not clear, although void nucleation is more likely to occur at intersections of grain boundaries with the substrate, and any perturbations or defects at the filmsubstrate interface. This is a field which requires further study, since the data available in the literature is sparse.

Regardless of whether solid-state dewetting is via grain boundary grooves or void growth from the interface, the final equilibrated state is that of a single crystalline particle on a substrate. If the initial film is thin enough, equilibrated particles will form within reasonable periods of time, and can be used to study the ECS of the film material [61, 93], and the interfacial energy between the film and the substrate $[56,58$, 94]. The kinetics of the dewetting process have also been used to extract surface diffusion parameters [95-97].

While fundamental studies of dewetting kinetics, and the final equilibrium configuration are important, dewetting has also been used as a method to pattern a surface with small particles for applications [81, 98-100]. Examples include catalysis [101-103], porous electrodes [104, 105], and more recently charge storage for memory applications [106-108].

\section{Thin film stability}

The discussion above leads to the necessary, albeit sometimes worrisome conclusion, that thin solid films are not stable. While this conclusion is trivial, it has important technological implications given the current dependence on thin film technology for the microelectronics industry. The minimization of total surface and interfacial energy is the driving force for dewetting, but the kinetics of the process depend on interface and surface transport mechanisms, and nucleation of the process may strongly depend on the nature and distribution of defects at the free surface, and at the interface between thin films and substrates. Given the advantages of particles with a length scale which influences their functional properties (i.e., "nano"), the last 10 years have seen a multitude of experiments designed to utilize 
(a)

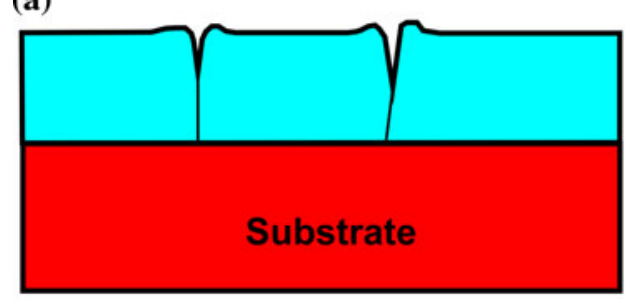

(c)

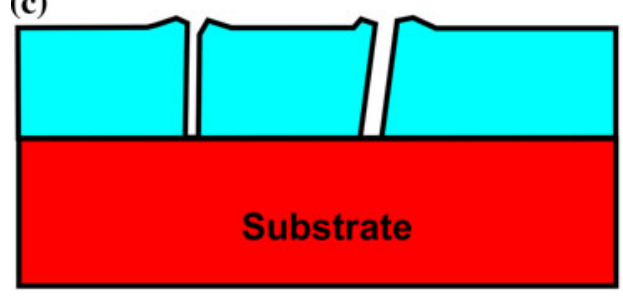

(e)

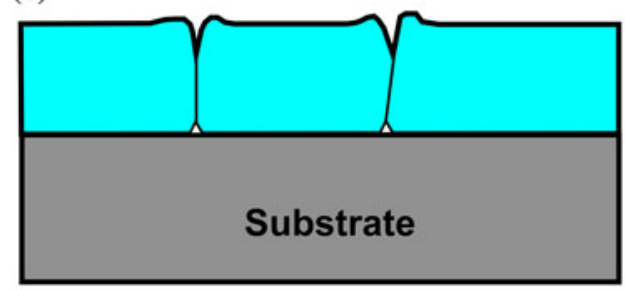

(g)

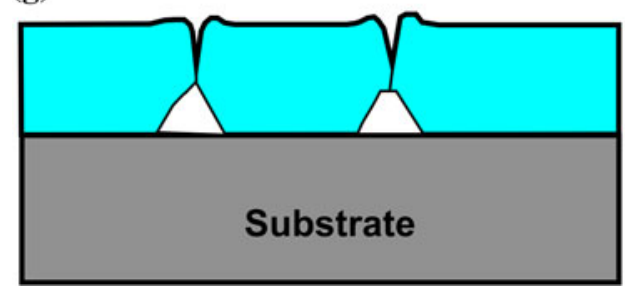

(b)

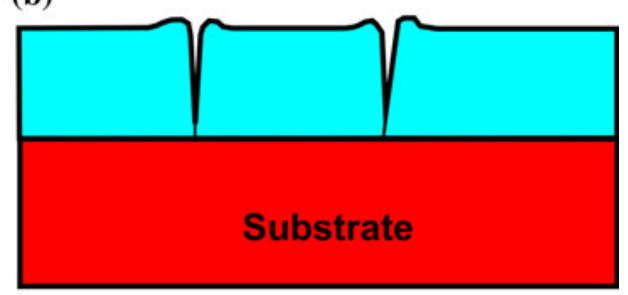

(d)

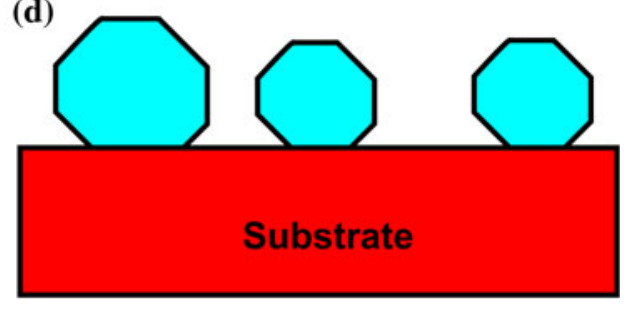

(f)

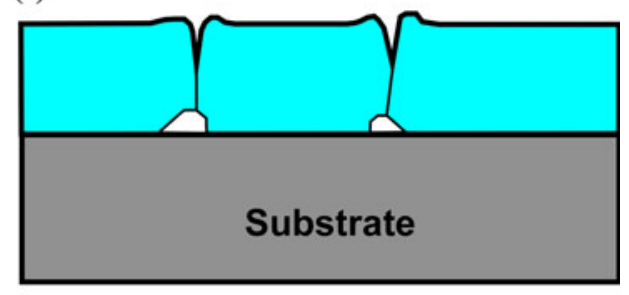

(h)

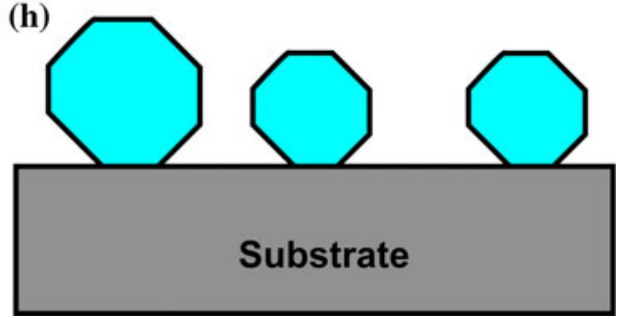

Fig. 14 Schematic drawing of solid-state dewetting of a thin film on a substrate, where a-d grain boundary grooves from the free surface slowly increase until they contact the substrate, and $\mathbf{e}-\mathbf{h}$ voids at triple junctions nucleate and grow towards the free surface

dewetting for this purpose. However, time-temperature experiments to probe the dewetting of continuous thin film devices, which depends on the defect microstructure, are lacking.

Precursor wetting foot and kinetics of spreading

A great deal of work has been done on the influence of the triple line during spreading of a liquid on a solid, or retraction of a liquid drop (liquid-state dewetting), and the associated hygrodynamics [30, 109]. The fluid dynamics involved are beyond the scope of the present work, but we would like to clarify the context of wetting versus spreading.

It is important to clarify the concept of a precursor foot, which is an undefined amount of the component of the liquid drop which extends upon the substrate ahead the main mass of the spreading drop [110] (see Fig. 15). A precursor foot can be either a bulk liquid phase identical to the drop phase, or an adsorption layer [111-114]. The expansion of the former is driven by macroscopic phenomena related to fluid mechanics and corresponds to the achievement of complete wetting, while the latter is driven by atomistic mechanisms and is related to the equilibration of the chemistry and the structure of the bare substrate at coexistence with the phase of the drop, and it has nothing to do with the wetting of a phase $[112,113]$. As we will see in subsequent sections of this review, an adsorption layer is an intrinsic part of a surface (or an interface) which is not a bulk phase. It is a region of finite thickness at a surface (or an interface) which contains all the gradients of composition and/or structure perpendicular to the surface which are necessary to minimize the surface (or interfacial) energy. 
(a)

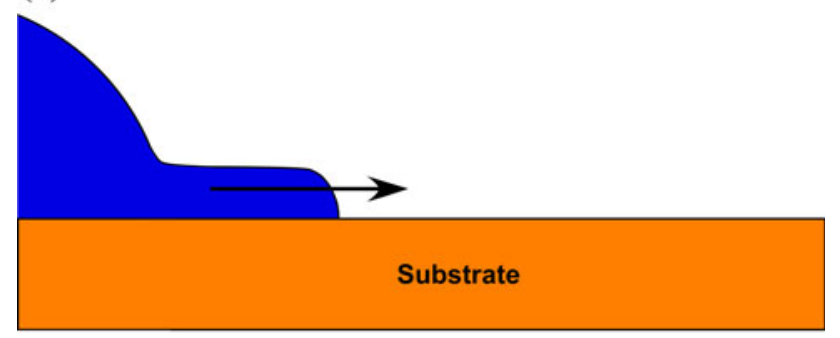

(b)

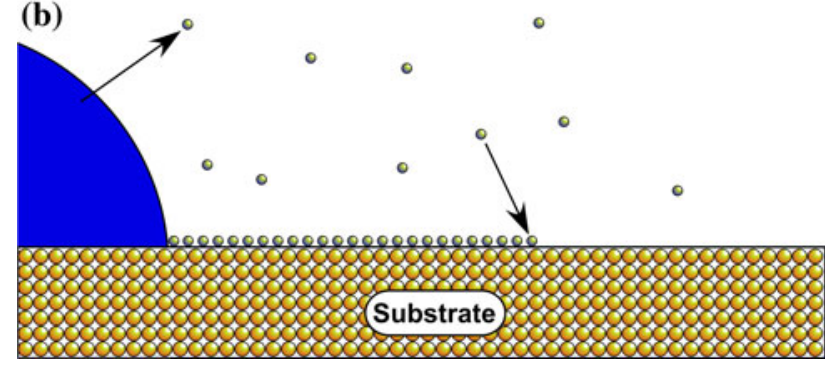

Fig. 15 Schematic drawing of a spreading drop with a a bulk precursor foot of liquid which precedes the main body, and $\mathbf{b}$ an adsorbate of atoms on the surface of the substrate which either diffused over the surface from the bulk drop, or evaporated from the bulk and then adsorbed to the surface from the gas phase

To form the adsorption layer, the constituent atoms from the drop either diffuse across the solid surface or evaporate and then adsorb onto the surface. In the thermodynamic regime, the contact angle reflects the modified (lowered) solid-vapor surface energy, while in the kinetic regime the driving force for spreading reflects the instantaneous surface energy of the substrate.

Due to the combined technological and fundamental importance of spreading rates, a great deal of fundamental and phenomenological research has been invested in this topic. For 'high' temperature materials science, two main regimes of spreading kinetics have been identified, for reactive and non-reactive systems.

The classical "low-temperature" spreading kinetics usually employs the hydrodynamic theory, where the spreading kinetics depends on the dynamic contact angle $\theta_{\mathrm{D}}$, and the capillary number $C_{a}=\nu \eta / \gamma_{\mathrm{LV}}\left(\gamma_{\mathrm{LV}}\right.$ is the surface energy of the liquid, $v$ is the velocity of the triple junction and $\eta$ is the liquid viscosity) [115]. The rate of spreading is thus reflected by the capillary number which contains the velocity of the triple line:

$$
\begin{aligned}
C_{a} & =\frac{\left(\theta_{\mathrm{D}}^{3}-\theta^{3}\right)}{9 \ln \frac{L_{\mathrm{c}}}{L_{\mathrm{S}}}} \quad\left(\theta_{\mathrm{D}} \leq 135^{\circ}\right) \\
C_{a} & =\frac{\left[\frac{9 \pi}{4} \ln \left(\frac{1-\cos \theta_{\mathrm{D}}}{1+\cos \theta_{\mathrm{D}}}\right)\left(\pi-\theta_{\mathrm{D}}\right)^{3}-\theta^{3}\right]}{9 \ln \frac{L_{\mathrm{c}}}{L_{\mathrm{S}}}} \quad\left(\theta_{\mathrm{D}} \leq 135^{\circ}\right)
\end{aligned}
$$

where $\theta$ is the equilibrium contact angle, $L_{\mathrm{c}}$ is a characteristic capillary length (Eq. 2 ), and $L_{\mathrm{S}}$ corresponds to a thickness of the meniscus immediately adjacent to the solid wall over which the 'no-slip' boundary condition of classical hydrodynamics is relaxed to avoid a singularity at the triple junction.

The molecular kinetic theory (MKT) of spreading was developed by Blake [116]. It assumes that the atoms of the spreading fluid replace adsorbed atoms on the surface, and yields:

$C_{a}=\frac{2 \eta \lambda k T}{h \gamma_{\mathrm{LV}}}\left[\sinh \left(\frac{\lambda^{2} \gamma_{\mathrm{LV}}}{2 k T}\left(\cos \theta-\cos \theta_{\mathrm{D}}\right)\right)\right] \exp \left(\frac{\Delta G_{\mathrm{w}}}{N k T}\right)$

where $\lambda$ is the average spacing between adsorption sites, $k$ is Boltzmann's constant, $h$ is Planck's constant, $\Delta G_{\mathrm{w}}$ is the activation free energy for wetting that derives mainly from solid-fluid interactions, $N$ is Avogadro's number, and $T$ is temperature.

Both approaches were developed for non-reactive spreading, and the last 10 years have seen numerous experiments designed to probe which approach best describes high temperature spreading of liquid metals (for a review of the subject see [117]). From meticulous experiments $[115,118]$ and molecular dynamics simulations [119-121], it appears that the rate limiting mechanism for non-reactive and dissolutive spreading kinetics is friction at the triple junction, where order in the liquid at the solidliquid interface plays a key role in defining this parameter. Order in the liquid at solid-liquid interfaces has been theoretically analyzed [122-129] and experimentally observed [130-135], and will be discussed below in terms of adsorption.

\section{Microscopic scale and adsorption}

Thus far we have discussed wetting under conditions where the compositions of the wetting and substrate phases have not been addressed explicitly. The compositions of the two condensed phases (and the vapor phase), and those of the interfacial regions in particular, are important, as we shall see, because changes in interfacial chemical compositions may cause changes in the interfacial energies that determine wetting equilibrium (see the Young, Neumann, and Herring wetting equilibrium equations described in sections "Macroscopic wetting of a liquid on a rigid solid substrate", "Wetting on unconstrained isotropic substrates", and "Effects of interfacial torques on wetting equilibrium").

This section introduces some basic concepts on the thermodynamics of interfaces that are useful for treating the chemistry of interfaces, and then proceeds to couple this formalism with a more atomistic approach. The more general concept of complexions, which includes order 
parameters in addition to composition, will be addressed at the end of this paper.

In this section of the paper, we follow the approach developed by Gibbs [136]. In a later section, we also introduce another formalism, due to Cahn, which is equivalent to Gibbs' treatment, but which is more transparent when dealing with multi-component, multi-phase systems that simultaneously contain several different interfaces. Gibbs is not easy to read, and so it is convenient to draw on other sources which have "translated" Gibbs' work into a more modern thermodynamic language, such as the papers by Hirth [137, 138] and by Mullins [41], and the book by Rowlinson and Widom [139]. Parts of what is covered in this paper on the topic of interfacial thermodynamics has been excerpted from a recent review of interfacial adsorption (or segregation) [140].

We begin by reviewing the thermodynamics of interfaces, using the liquid-vapor surface as an example, i.e., we consider a system composed of a liquid in equilibrium with a vapor phase, separated by an interface. The energy associated with the interface, i.e., the surface or interfacial energy, denoted by $\gamma$ (units of [energy/(unit area)], typically $\mathrm{mJ} / \mathrm{m}^{2}$ ), is defined as the reversible work needed to create unit area of surface, at constant temperature $(T)$, volume $(V)$ (or pressure $(P)$ ), and chemical potentials of the components $i$ of the system $\left(\mu_{i}\right)$.

\section{Gibbs dividing surface}

The interface is not perfectly sharp and has a finite thickness, i.e., there is a transition region separating the two phases where the local density changes from that of the liquid (referred to as phase ') to that of the vapor (referred to as phase "), as shown schematically in Fig. 16. The diffuseness illustrated here for a liquid/vapor interface is also present at other types of interfaces, such as grain boundaries, solid-liquid, or solid-solid interphase boundaries. Note that the gradient in composition, and in other kinds of density parameters, can vary in shape and width (see section "Complexions"). In order to avoid having to define the diffuseness of the interface, Gibbs defined a mathematical plane, known as the dividing surface, with area $A$, onto which he projected all the extensive thermodynamic variables pertaining to the interface. To do this, he took the value of the variable in the system containing the interface, and subtracted the value of the variable obtained from a hypothetical reference system consisting of the two bulk phases, assumed to extend uniformly all the way to this mathematical plane. An extensive variable obtained by this subtraction process is referred to as an interfacial excess quantity, and is identified by the superscript S. As

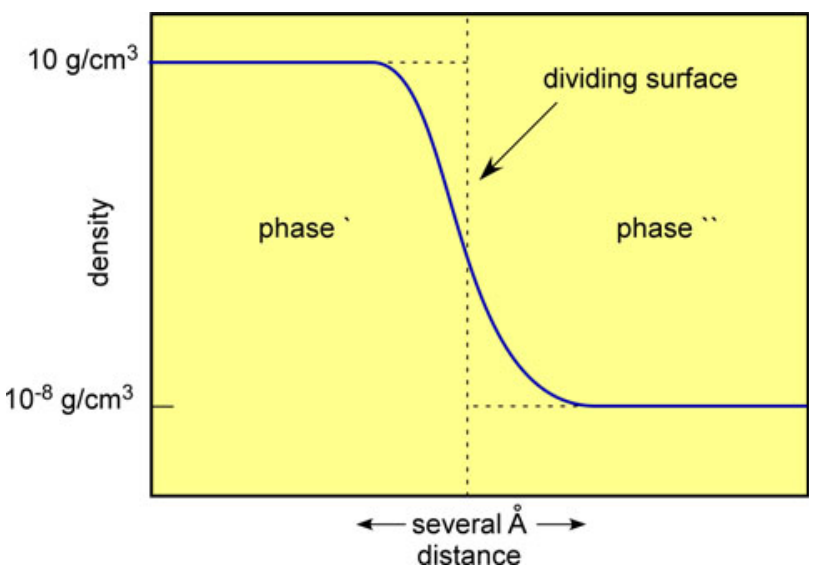

Fig. 16 Schematic of the density variation across a liquid-vapor interface. The vertical dashed line represents the Gibbs' dividing surface. The solid line represents the density variation in the system containing the interface. Horizontal dashed lines illustrate the definition of the hypothetical reference system

an example, the interfacial excess internal energy of the system, $E^{\mathrm{S}}$, may be written as:

$E^{\mathrm{S}}=E-E^{\prime}-E^{\prime \prime}$

where $E$ is the internal energy of the system (containing the interface), $E^{\prime}$ is the internal energy of phase ', and $E^{\prime \prime}$ is the internal energy of phase ". This shows how the interfacial excess quantity is just the quantity in the total system, less the quantity in the bulk phases (hypothetically extended to the dividing surface). It is important to note that the position of the dividing surface is arbitrary. It can be placed at any convenient location within the bounds of the physically diffuse region. Some consequences of this arbitrariness are addressed below.

Every extensive thermodynamic variable of the system can be written in the same manner as in Eq. 18; e.g., the number of moles of component $i$ in the real system is expressed as: $n_{i}=n_{i}^{\prime}+n_{i}^{\prime \prime}+n_{i}^{\mathrm{s}}$. The only exception is the volume of the system, which is written:

$V^{\mathrm{S}}=V^{\prime}-V^{\prime \prime}$

i.e., Gibbs assigned no excess volume to the interface.

\section{Panel 3: inclusion of surface energy into the standard expression for internal energy}

The internal energy of any bulk phase (say phase ') is conveniently considered to be a function of the extensive variables entropy, $S^{\prime}$, volume, $V^{\prime}$ and number of moles, $n_{i}^{\prime}$ : 
$\mathrm{d} E^{\prime}=\left(\frac{\partial E^{\prime}}{\partial S^{\prime}}\right)_{V^{\prime}, n_{i}^{\prime}} \mathrm{d} S^{\prime}+\left(\frac{\partial E^{\prime}}{\partial V^{\prime}}\right)_{S^{\prime}, n_{i}^{\prime}} \mathrm{d} V^{\prime}+\sum_{i}\left(\frac{\partial E^{\prime}}{\partial n_{i}^{\prime}}\right)_{S^{\prime}, V^{\prime}, n_{i \neq i}^{\prime}} \mathrm{d} n_{i}^{\prime}$

(P3(1)20)

or

$\mathrm{d} E^{\prime}=T \mathrm{~d} s^{\prime}-P \mathrm{~d} V^{\prime}+\sum_{i} \mu_{i}^{\prime} d n_{i}^{\prime}$

where $T$ is the temperature, $P$ is the pressure, and $\mu_{i}^{\prime}$ is the chemical potential of component $i$ in phase' . This is the standard expression for the internal energy of a single uniform phase containing more than one component. Similarly, the internal energy of phase " is:

$\mathrm{d} E^{\prime \prime}=T \mathrm{~d} S^{\prime \prime}-P \mathrm{~d} V^{\prime \prime}+\sum_{i} \mu_{i}^{\prime \prime} \mathrm{d} n_{i}^{\prime \prime}$

For the surface (which has an area, $A$, but zero excess volume) the excess internal energy is written:

$\mathrm{d} E^{\mathrm{S}}=T \mathrm{~d} S^{\mathrm{S}}+\gamma \mathrm{d} A+\sum_{i} \mu_{i}^{\mathrm{S}} \mathrm{d} n_{i}^{\mathrm{S}}$

This illustrates that in the expression for the surface excess internal energy, the $P \mathrm{~d} V$ term is replaced by a term consisting of the surface energy, $\gamma$, multiplied by a change in area, $\mathrm{d} A$. Since, for a system in equilibrium, the chemical potential must be equal everywhere, we impose the condition: $\mu_{i}^{\prime}=\mu_{i}^{\prime \prime}=\mu_{i}^{\mathrm{s}}$. Substituting for $\mathrm{d} E^{\prime}, \mathrm{d} E^{\prime \prime}$ and $\mathrm{d} E^{\mathrm{S}}$ into Eq. (18), we obtain:

$$
\begin{aligned}
\mathrm{d} E= & T\left(\mathrm{~d} S^{\prime}+\mathrm{d} S^{\prime \prime}+\mathrm{d} S^{\mathrm{S}}\right)-P\left(\mathrm{~d} V^{\prime}+\mathrm{d} V^{\prime \prime}\right)+\gamma \mathrm{d} A \\
& +\sum_{i} \mu_{i}\left(\mathrm{~d} n_{i}^{\prime}+\mathrm{d} n_{i}^{\prime \prime} \mathrm{d} n_{i}^{\mathrm{s}}\right)
\end{aligned}
$$

or

$\mathrm{d} E=T \mathrm{~d} S-P \mathrm{~d} V+\gamma \mathrm{d} A+\sum_{i} \mu_{i} \mathrm{~d} n_{i}$

This is the standard expression for the differential of the internal energy of a system containing an interface. All differentials in Eq. (P3(6)25) pertain to extensive variables, so the equation can readily be integrated to yield:

$E=T S-P V+\gamma A+\sum_{i} \mu_{i} n_{i}$

(P3(7)26)

"Panel 3" shows that the internal energy of a system with an interface may be expressed as in Eqs. (P3(6)25)(P3(7)26). This is an important result, as it demonstrates that in the limit of a system where the contribution of the surface (or interfacial) energy is small with respect to the bulk energy, the $\gamma \mathrm{d} A$ term becomes negligible and the expression reduces to the standard thermodynamic expression for the internal energy of a bulk phase. All other thermodynamic variables of the system containing a surface can be derived from these expressions.
For example, the Helmholtz free energy is given by:

$F=E-T S$

where $S$ is the entropy, and the derivative of $F$ (when combined with Eq. P3(6)25) may be written:

$\begin{aligned} \mathrm{d} F= & \mathrm{d} E-T \mathrm{~d} S-S \mathrm{~d} T=-S \mathrm{~d} T-P \mathrm{~d} V+\gamma \mathrm{d} A \\ & +\sum_{i} \mu_{i} \mathrm{~d} n_{i}\end{aligned}$

so that:

$\mathrm{d} F^{\mathrm{S}}=-S^{\mathrm{S}} \mathrm{d} T+\gamma \mathrm{d} A+\sum_{i} \mu_{i} \mathrm{~d} n_{i}^{S}$

Whereas the Helmholtz free energy is an appropriate thermodynamic function for a closed system (as is the Gibbs free energy), the grand potential, $\Omega$, is a form of free energy that is suitable for open systems [139]. It is defined as:

$\Omega=F-\sum_{i} \mu_{i} n_{i}=-P V+\gamma A$

where we have used Eq. (P3(7)26) and Eq. (27) to obtain the second equality. Thus, the surface excess grand potential may be expressed as:

$\Omega^{\mathrm{S}}=\Omega-\Omega^{\prime}-\Omega^{\prime \prime}=-P V+\gamma A+P\left(V^{\prime}+V^{\prime \prime}\right)=\gamma A$

This expression shows that the surface energy is just the surface excess grand potential per unit area. In particular, $\Omega^{\mathrm{S}}$ is a useful quantity, as Eq. (31) demonstrates that it is independent of any excess quantities that depend on the location of the dividing surface.

\section{Gibbs adsorption equation}

We now return to Eq. (P3(4)23), in which $\mathrm{d} E^{\mathrm{S}}$ is expressed only in terms of extensive variables $\left(\mathrm{d} S^{\mathrm{S}}, \mathrm{d} A, \mathrm{~d} n_{i}^{\mathrm{S}}\right)$ while the intensive variables $\left(T, \gamma, \mu_{i}\right)$ are constant in the equilibrated system. This can be integrated to obtain:

$E^{\mathrm{S}}=T S^{\mathrm{S}}+\gamma A+\sum_{1} \mu_{i} n_{i}^{\mathrm{S}}$

Re-differentiating this result yields:

$\mathrm{d} E^{\mathrm{S}}=T \mathrm{~d} S^{\mathrm{S}}+S^{S} \mathrm{~d} T+\gamma \mathrm{d} A+A \mathrm{~d} \gamma+\sum_{i} \mu_{i} \mathrm{~d} n_{i}^{\mathrm{S}}+\sum_{i} n_{i}^{\mathrm{S}} \mathrm{d} \mu_{i}$

Subtracting Eq. (P3(4)23) from Eq. (33), we obtain:

$S^{\mathrm{S}} \mathrm{d} T+A \mathrm{~d} \gamma+\sum_{i} n_{i}^{\mathrm{S}} \mathrm{d} \mu_{i}=0$

We now define the following new quantities: $s^{\mathrm{S}} \equiv S^{\mathrm{S}} / A$, and $\Gamma_{i} \equiv n_{i}^{\mathrm{S}} / A$. Quantities such as $s^{\mathrm{S}}$ and $\Gamma_{i}$, expressed per 
unit area, are known as specific interfacial excess quantities, e.g., $s^{\mathrm{S}}$ is known as the specific interfacial excess entropy, and $\Gamma_{i}[\mathrm{~mol} /$ (unit area)] is the specific interfacial excess number of moles of component $i$. However, in order to avoid this cumbersome nomenclature, $\Gamma_{i}$ is generally referred to simply as the adsorption of component $i$. With these definitions we can rewrite Eq. (34) as:

$\mathrm{d} \gamma=-s^{\mathrm{s}} \mathrm{d} T-\sum_{i} \Gamma_{i} \mathrm{~d} \mu_{i}$

Equation (35) is the well-known Gibbs adsorption equation. It gives the variation of $\gamma$ with changes in $T$ and $\mu_{i}$, where the $\mu_{i}$ are related to the compositions of the bulk phases. At constant temperature, this equation simplifies to the Gibbs adsorption isotherm. In the case of a two-component A-B system, we can write:

$\mathrm{d} \gamma=-\Gamma_{\mathrm{A}} d \mu_{\mathrm{A}}-\Gamma_{\mathrm{B}} \mathrm{d} \mu_{\mathrm{B}}$

where the subscripts A and B stand for the components. By convention, the solvent (majority component) is labeled A and the solute (minority component) is labeled B. However, the chemical potentials of the two components are not independent variables; they are related by the Gibbs-Duhem equations that can be written for each of the two bulk phases. For example, for phase':

$n_{\mathrm{A}}^{\prime} \mathrm{d} \mu_{\mathrm{A}}+n_{\mathrm{B}}^{\prime} \mathrm{d} \mu_{\mathrm{B}}=0$

which is valid either at constant $T$ and $P$, or at constant $T$ and $V$. Equation (37) can be used to eliminate one of the chemical potentials in Eq. (36). By convention, the chemical potential of the solvent is eliminated, and the Gibbs isotherm is rewritten in terms of the solute:

$\frac{\mathrm{d} \gamma}{\mathrm{d} \mu_{\mathrm{B}}}=\Gamma_{\mathrm{A}} \frac{n_{\mathrm{B}}^{\prime}}{n_{\mathrm{A}}^{\prime}}-\Gamma_{\mathrm{B}}$

Here, $\mathrm{d} \gamma / \mathrm{d} \mu_{\mathrm{B}}$ is a measurable quantity, and therefore cannot depend on an arbitrary choice of the dividing surface. On the other hand, $\Gamma_{\mathrm{A}}$ and $\Gamma_{\mathrm{B}}$ do depend on the position of the dividing surface. Thus, the right-hand side of Eq. (38): $\Gamma_{\mathrm{A}} \frac{n_{\mathrm{B}}^{\prime}}{n_{\mathrm{A}}^{\prime}}-\Gamma_{\mathrm{B}}$ (i.e., this particular combination of adsorption variables) must also be independent of the position of the dividing surface.

In the case of interfaces between a condensed phase and a vapor phase, the difference in density across the interface makes it possible to select the position of the Gibbs dividing surface so as to make one of the adsorption terms vanish. In those cases, Eq. (38) can be simplified by choosing the position of the dividing surface so as to make $\Gamma_{\mathrm{A}}=0$. Under these conditions, $\Gamma_{\mathrm{B}}$ is no longer arbitrary (since a specific choice for the position of the dividing surface has been made) and is written $\Gamma_{\mathrm{B}(\mathrm{A})}$ to indicate the choice $\Gamma_{\mathrm{A}}=0$ :

$\frac{\mathrm{d} \gamma}{\mathrm{d} \mu_{\mathrm{B}}}=-\Gamma_{\mathrm{B}(\mathrm{A})}$

$\Gamma_{\mathrm{B}(\mathrm{A})}$ is sometimes referred to as the relative adsorption [141]. The value of $\Gamma_{\mathrm{B}(\mathrm{A})}$ (or of any adsorption term) can be either positive or negative. From Eq. (39), it can be seen that if the relative adsorption of component B is positive (i.e., there is a positive excess of component $B$ at the surface of the condensed phase) then $\mathrm{d} \gamma / \mathrm{d} \mu_{\mathrm{B}}$ will be negative, and the surface energy of the solid will decrease as the chemical potential (i.e., the bulk concentration) of component B is increased. However, the simplification shown here for the case of interfaces between a condensed phase and a vapor cannot generally be applied to grain boundaries, or other interfaces between two condensed phases. Thus, in those cases, the variation of surface energy with chemical potential may be obtained from expressions based on the grand potential (e.g., Eqs. 30, 31), which do not depend on the position of the dividing surface. Some useful approximations for the Gibbs adsorption isotherm are given in "Panel 4".

\section{Panel 4: useful approximate form of the Gibbs adsorption isotherm}

The term, $\mathrm{d} \mu_{\mathrm{B}}$, in Eqs. (38) or (39), can be transformed using the definition of chemical potential in terms of activity, $a: \mu_{\mathrm{B}}=\mu_{\mathrm{B}}{ }^{\circ}+k T \ln a_{\mathrm{B}}$, where $\mu_{\mathrm{B}}{ }^{\circ}$ is the chemical potential in the standard state. In the case of ideal solutions, Raoult's law can be applied: $a_{\mathrm{B}}=x_{\mathrm{B}}$, where $x_{\mathrm{B}}$ is the atom (or mole) fraction of the solute. In dilute solutions, one may apply Henry's law: $a_{\mathrm{B}}=k_{\mathrm{o}}$ $x_{\mathrm{B}}$, where $k_{\mathrm{o}}$ is a constant. In either case, $\mathrm{d} \mu_{\mathrm{B}}=R T$ $\mathrm{d}\left(\ln x_{\mathrm{B}}\right)$. Therefore, for ideal or Henrian solutions, the above relationships may be simplified as follows:

$\frac{1}{R T} \frac{\mathrm{d} \gamma}{\mathrm{d} \ln x_{\mathrm{B}}}=\left(\Gamma_{\mathrm{A}} \frac{n_{\mathrm{B}}^{\prime}}{n_{\mathrm{A}}^{\prime}}-\Gamma_{\mathrm{B}}\right)$

or

$\frac{1}{R T} \frac{\mathrm{d} \gamma}{\mathrm{d} \ln x_{\mathrm{B}}}=-\Gamma_{\mathrm{B}(\mathrm{A})}$

Thus, we can obtain the adsorption, $\Gamma_{\mathrm{B}(\mathrm{A})}$ from the dependence of the surface energy on bulk composition. In principle, one can also obtain the change in surface energy that results from the adsorption of solute. To do this, it is necessary to integrate the Gibbs isotherm, which requires some knowledge of the dependence of adsorption on $\mu_{\mathrm{B}}$ or $x_{\mathrm{B}}$. Unfortunately, Gibbsian thermodynamics do not provide this dependence. In 
section "Microscopic scale and adsorption", we describe a simple model that gives an approximate form for the needed functional relationship. In addition, that section provides some simple examples that illustrate the trends expected from this formalism.

Now that adsorption has been defined, it is useful to briefly discuss the relationship between interfacial segregation and adsorption. This issue has been discussed previously, for example, by Hondros and Seah [142]. Historically, the term adsorption was first used to describe the presence of excess components at the surface of a condensed phase, which originated from the gas phase, as shown in Fig. 17a, b. In contrast, the term segregation was used to describe excess components at a surface that originated from the bulk of the

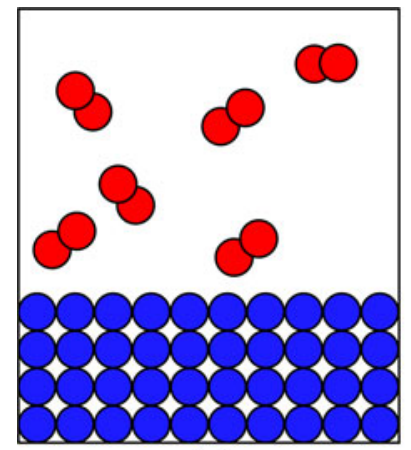

(a)

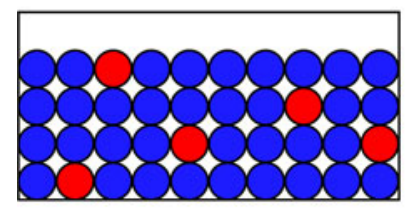

(c)

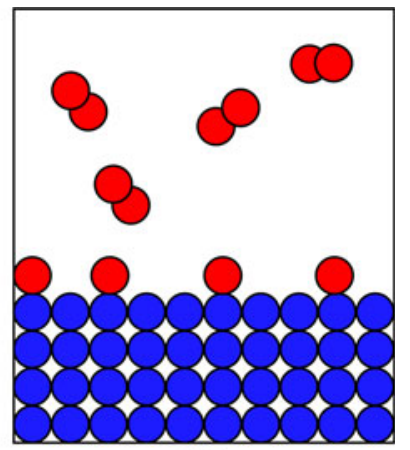

(b)

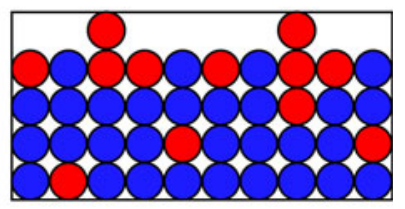

(d)

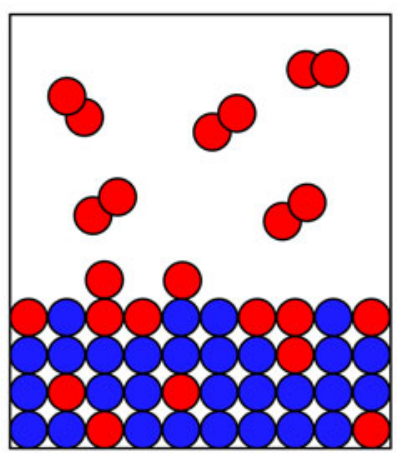

(e)

Fig. 17 a Initial state of a system consisting of a condensed phase with a surface exposed to a gas environment of diatomic molecules of red species; $\mathbf{b}$ state of system $\mathbf{a}$ after some of the gas molecules have adsorbed onto the surface and dissociated. $\mathbf{c}$ Initial state of a system containing some of the red species dissolved in the bulk; $\mathbf{d}$ state of system $\mathbf{c}$ after some of the dissolved red species have segregated to the surface. e Equilibrium state of the systems of figures a-d showing the gas phase, the equilibrium solution of the red species in the bulk of the condensed phase, and the equilibrated surface with adsorbed/segregated red species (Color figure online) condensed phase, as shown in Fig. 17c, d. In a system at equilibrium, consisting of a condensed phase in contact with a gas phase, the quantity of adsorbed or segregated component at the interface is independent of its origin (i.e., gas or bulk condensed phases). The final equilibrium state of such a system is illustrated in Fig. 17e. For our purposes, therefore, at equilibrium, the terms adsorption or segregation may be considered to be identical. However, it should be noted that the ways in which adsorption energy and segregation energy are defined are not identical.

Illustration of adsorption/segregation by means of a simple model

\section{Model framework}

Many models have used the concepts of statistical thermodynamics to develop expressions that relate interfacial adsorption to the bulk compositions of the adjacent phases [140, 141, 143-147]. Here, we make use of the model of Wynblatt and Chatain [140] for some simple illustrations.

Consider a system consisting of a two-component crystalline phase terminated by a surface of given crystallographic orientation. In general, the composition of the near-surface region will be different from that of the bulk, i.e., the surface will be enriched in one of the two components due to segregation from the bulk. In order to simplify matters, we assume that the enriched surface region is only one atom layer thick, i.e., we assume a "monolayer model", as illustrated in Fig. 18 for a (100) fcc surface. Although the model allows the enriched surface region to have any number of enriched layers, this simpler version will suffice for the present purposes of illustration.

The two-component crystalline bulk phase and its associated surface monolayer are each assumed to behave as regular solutions. In the regular solution approximation,

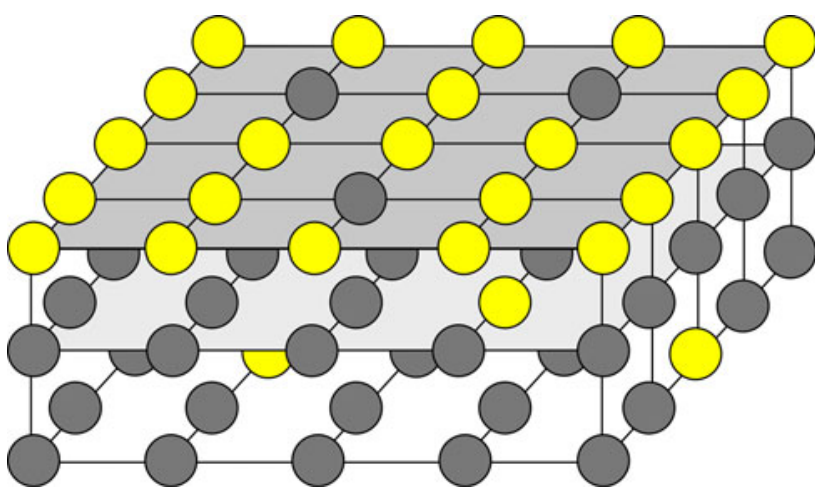

Fig. 18 Schematic of the monolayer model as applied to a twocomponent system with a (100) fcc surface: yellow circles represent atoms of the segregating species (chosen to be the solute). This species is enriched in the topmost atom layer of the surface. Darker circles represent the other species (Color figure online) 
the entropies of the bulk and surface solutions are taken to be the same as that of ideal solutions. However, unlike ideal solutions, a finite enthalpy or internal energy of mixing is allowed in both the bulk and the surface. The surface energy is first written as the surface excess grand potential, by combining Eqs. (27), (30), and (31)::

$\gamma A=\Omega^{\mathrm{S}}=F^{\mathrm{S}}-\sum_{i} \mu_{i} n_{i}^{\mathrm{S}}=E^{\mathrm{S}}-T S^{\mathrm{S}}-\sum_{i} \mu_{i} n_{i}^{\mathrm{S}}$

In order to write the various terms of Eq. (42) in the regular solution approximation, we need to define the following terms: $x^{\mathrm{S}}$ and $x$ are the atom fractions of the solute (component $\mathrm{B}$, assumed to be the segregating (adsorbing) component) in the interface and the bulk, respectively; $\gamma_{A}$ and $\gamma_{B}$ are the surface energies of the pure $\mathrm{A}$ and $\mathrm{B}$ components in the binary solution (component $\mathrm{B}$ taken to be the solute); and $\omega$ is the regular solution parameter for the $\mathrm{AB}$ solution. $\omega$ is defined as: $\omega=\varepsilon_{\mathrm{AB}}-$ $\left(\varepsilon_{\mathrm{AA}}+\varepsilon_{\mathrm{BB}}\right) / 2$, where $\varepsilon_{\mathrm{AB}}, \varepsilon_{\mathrm{AA}}$, and $\varepsilon_{\mathrm{BB}}$ (taken to be negative quantities) are the energies of bonds connecting $\mathrm{A}-\mathrm{B}, \mathrm{A}-\mathrm{A}$, and $\mathrm{B}-\mathrm{B}$ neighboring atom pairs, respectively. $z^{1}$ and $z^{\mathrm{v}}$ are the numbers of in-plane bonds and half of the out-of-plane bonds of an atom in the surface plane, such that the total coordination number of an atom in the bulk of the system is given by: $z=z^{1}+2 z^{\mathrm{v}}$. Finally, the surface excess energy and entropy are written as:

$$
\begin{aligned}
& E^{\mathrm{S}}= \frac{N z^{1}}{2}\left\{\left(x^{\mathrm{S}}\right)^{2} \varepsilon_{\mathrm{BB}}+2 x^{\mathrm{S}}\left(1-x^{\mathrm{S}}\right) \varepsilon_{\mathrm{AB}}+\left(1-x^{\mathrm{S}}\right)^{2} \varepsilon_{\mathrm{AA}}\right\} \\
&+N z^{\mathrm{v}}\left\{x^{\mathrm{S}} x \varepsilon_{\mathrm{BB}}+x^{\mathrm{S}}(1-x) \varepsilon_{\mathrm{AB}}+x\left(1-x^{\mathrm{S}}\right) \varepsilon_{\mathrm{AB}}\right\} \\
&+\left.(1-x)\left(1-x^{\mathrm{S}}\right) \varepsilon_{\mathrm{AA}}\right\}-N\left(\frac{z^{1}}{2}+\frac{3}{2} z^{\mathrm{v}}\right) \\
&\left\{x^{2} \varepsilon_{\mathrm{BB}}+2 x(1-x) \varepsilon_{\mathrm{AB}}+(1-x)^{2} \varepsilon_{\mathrm{AA}}\right\}
\end{aligned}
$$

$$
\begin{aligned}
S^{S}= & -N R\left\{x^{\mathrm{S}} \ln x^{\mathrm{S}}+\left(1-x^{\mathrm{S}}\right) \ln \left(1-x^{\mathrm{S}}\right)\right\} \\
& -\{x \ln x+(1-x) \ln (1-x)\}
\end{aligned}
$$

where $N$ is the number of surface atom per unit area.

By minimizing $\gamma$ with respect to $x^{\mathrm{S}}$ [140], it is possible to obtain the following expression for the equilibrium value of the segregated monolayer composition, $x^{\mathrm{s}}$ :

$\frac{x^{\mathrm{S}}}{1-x^{\mathrm{S}}}=\frac{x}{1-x} \exp -\frac{\Delta E_{\text {seg }}}{R T}$

where

$$
\begin{aligned}
\Delta E_{\mathrm{seg}}= & \left(\gamma_{\mathrm{B}}-\gamma_{\mathrm{A}}\right) \sigma+2 \omega\left[z^{1}\left(x-x^{\mathrm{S}}\right)+z^{v}\left(x-\frac{1}{2}\right)\right] \\
& -\Delta E_{\mathrm{el}}
\end{aligned}
$$

$\Delta E_{\mathrm{el}}$ is the change in elastic strain energy experienced by a solute atom when it segregates from the bulk to the surface, and $\sigma$ is the area per mole at the (monolayer) interface. $\Delta E_{\text {el }}$ may be expressed, for example, by a relation due to Friedel [148] as:

$\Delta E_{\mathrm{el}}=\frac{24 \pi K_{\mathrm{B}} G_{\mathrm{A}} r_{\mathrm{B}}\left(r_{\mathrm{A}}-r_{\mathrm{B}}\right)^{2}}{3 K_{\mathrm{B}} r_{\mathrm{B}}+4 G_{\mathrm{A}} r_{\mathrm{A}}}$

where $K_{\mathrm{B}}$ is the bulk modulus of the solute, $G_{\mathrm{A}}$ is the shear modulus of the solvent, and $r_{\mathrm{B}}$ and $r_{\mathrm{A}}$ are the atomic radii of the pure solute and solvent atoms, respectively.

It should be noted that $x^{\mathrm{S}}$, the atom fraction of solute (B) in the segregated monolayer, is not identical with the Gibbsian $\Gamma$. The relation between these two quantities is:

$\Gamma_{\mathrm{B}}=\left(x^{\mathrm{S}}-x\right) / \sigma$

Such expressions were used to relate surface atom fractions to the adsorption of the various components in Eq. (42) in order to obtain Eqs. (44) and (45) by minimization of $\gamma$ with respect to $x^{\mathrm{S}}$. Some physical characteristics of the energy of segregation are given in "Panel 5".

Panel 5: contributions to the driving force for surface segregation in the regular solution model

The model of Eqs. (44) and (45) shows that there are three principal contributions responsible for segregation to surfaces (or grain boundaries) in alloys. There is a "chemical" driving force, which consists of two terms: one of which depends on the difference between the surface energies of the pure components (i.e., a surface energy driving force) and another that depends on the regular solution constant (i.e., an interatomic interaction driving force). The third driving force is an elastic strain energy contribution associated with the degree of misfit of the solute in a solid solution. Both the regular solution constant term and the elastic strain energy term vanish in the limit of an ideal solution (for which $\omega=0$, and the atoms must necessarily have nearly identical size).

The extent of segregation depends not only on the magnitude of the three contributions mentioned above, but also on their signs. Negative contributions to the heat of segregation increase the value of $x^{\mathrm{S}}$ in Eq. (44), whereas positive ones decrease $x^{\mathrm{S}}$. Since $\Delta E_{\text {el }}$ (Eq. 46) is always positive, the solute strain energy contribution to the heat of segregation always favors solute segregation. In contrast, the two chemical terms can be either positive or negative for the solute. The surface energy of the pure solute can either be greater or smaller than that of the solvent, and a smaller surface energy will tend to 
promote adsorption/segregation of that component. Similarly, a positive regular solution constant, $\omega$, will add to the driving force for solute segregation. Thus, it is the sign of $\Delta E_{\mathrm{seg}}$, arising from the sum of the three contributions, that will determine whether the solute or the solvent will tend to segregate to the interface. Furthermore, solute segregation will be strongest when all three contributions are negative [147].

\section{Expected trends}

Figure 19a is a plot of $\ln \left(x^{\mathrm{S}} / 1-x^{\mathrm{S}}\right)$ (i.e., the logarithm of the left-hand side of Eq. 44) versus $1 / T$, obtained by applying the monolayer model to the (100) surface of a fcc solid solution, for various values of $\Delta E_{\mathrm{seg}}$, as defined in Eq. (45). In the plots, the surface energies of the pure components and the solute elastic strain energy have been kept constant, but the regular solution parameter, $\omega$, has been changed from positive, to zero, to negative. Consideration of Eq. (44) shows that when $\omega=0, \Delta E_{\text {seg }}$ becomes independent of surface composition. As a result, the line in Fig. 19a corresponding to that condition is a straight line. For the other two cases, with finite $\omega$, the dependence of $\ln \left(x^{\mathrm{S}} / 1-x^{\mathrm{S}}\right)$ on $1 / T$ is no longer a straight line, i.e., its slope varies with temperature through the dependence of $x^{\mathrm{S}}$ on temperature. Furthermore, the figure shows that segregation is stronger for positive $\omega$ and weaker for negative $\omega$. Positive $\omega$ corresponds to solid solutions with a tendency to cluster, in which the solute (and solvent) atoms prefer to be surrounded by atoms of their own species. At suitable temperatures, this tendency will lead to bulk phase separation into an A-rich phase and a B-rich phase. This incipient tendency to phase separation means that once some solute atoms are driven to the surface (by the surface energy and/or elastic energy

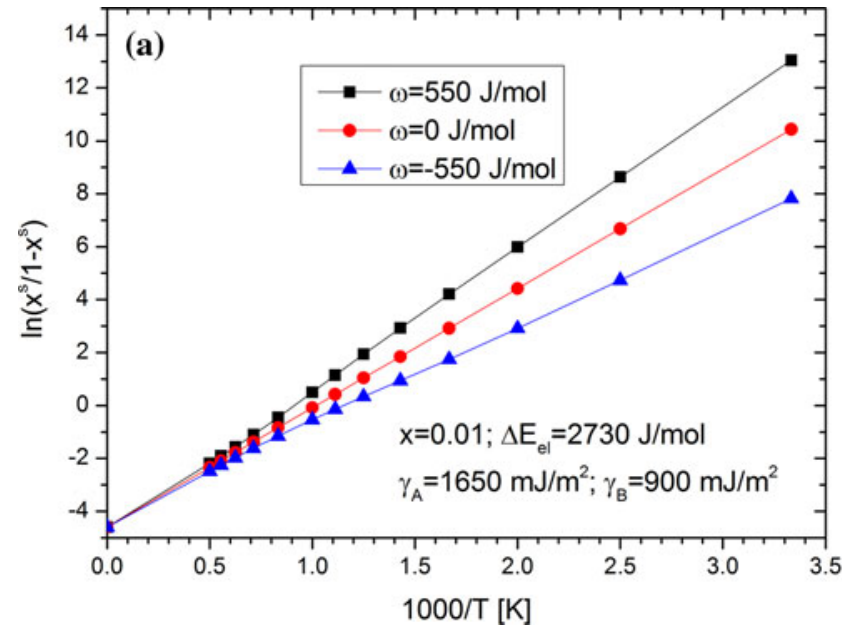

Fig. 19 a Dependence of (100) surface segregation on temperature for fcc solutions with a bulk atomic fraction of the solute component B, $x=0.01$, for the monolayer model computed by Eqs. (44)-(46). The solute strain energy and surface energy driving forces are held driving forces) then it becomes energetically favorable for more solute atoms to be present there. Conversely, for cases where $\omega$ is negative, the solution will tend to order, i.e., atoms of one species will prefer to be surrounded by atoms of the other species. This tendency makes high concentrations of like atoms at an interface energetically less favorable, thereby decreasing the strength of segregation. Also, note that the value of $\ln \left(x^{\mathrm{S}} / 1-x^{\mathrm{S}}\right)$, in the limit of infinite temperature $(1 / T=0)$, is identical for all three cases. From Eq. (44), it can be seen that as $1 / T$ tends to $0, \ln \left(x^{\mathrm{S}} / 1-x^{\mathrm{S}}\right)$ approaches $\ln (x / 1-x)$; i.e., segregation tends to vanish in the limit of high temperatures.

The monolayer model used to obtain Fig. 19a assumes that only the outermost surface layer can change its composition. The validity of this approximation is examined in Fig. 19b, which compares the results obtained from the monolayer model with results of a multilayer model in which all atomic layers are allowed to reach equilibrium [140]. In order to emphasize the effect of considering multilayers, the bulk composition was increased from $x=0.01$ in Fig. 19a to $x=0.1$ in Fig. 19b. The figure shows that the total adsorption with the more complete model is about $50 \%$ larger than with the monolayer model.

Integral form of the surface energy

Recall that it is not possible to relate the adsorption, $\Gamma$, to the chemical potential, $\mu$, within the Gibbsian framework of interfacial thermodynamics. This prevents integration of the Gibbs adsorption isotherm to obtain the change in interfacial energy resulting from adsorption. In the previous section, we have used a statistical thermodynamic approach to obtain interfacial composition, $x^{\mathrm{S}}$, as a

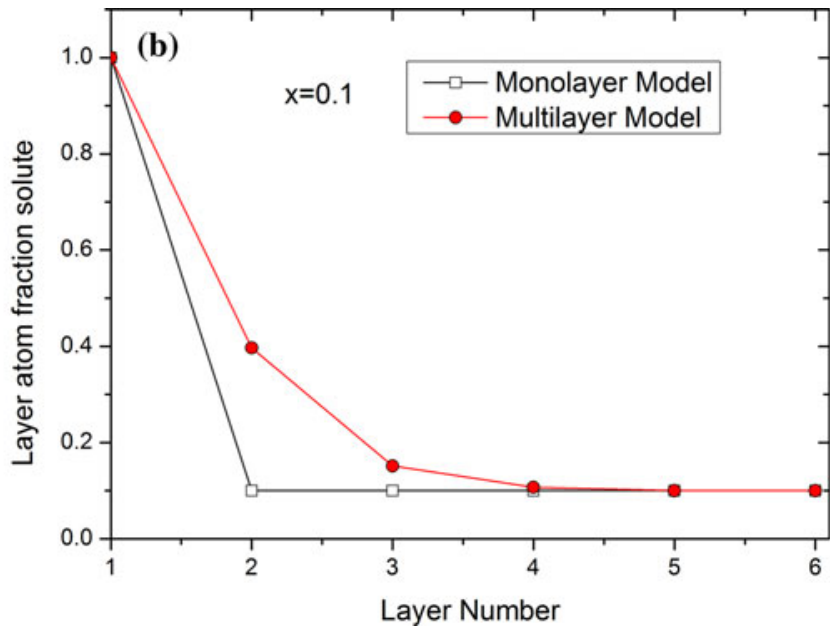

constant, but the regular solution constant, reflecting interatomic interactions, is varied. b Comparison of surface segregation profiles obtained by the monolayer and multilayer models, for a bulk compositions of $x=0.1$, with the same parameters as a 
function of bulk composition, $x$, in Eq. (44), and this information can be employed to obtain the surface energy for any given equilibrium state of adsorption.

In principle, it is easy to integrate the Gibbs adsorption isotherm, but only for the case $\omega=0$ (ideal solution) where $\Delta E_{\text {seg }}$ is independent of $x^{\mathrm{S}}$ (see Eq. 45), as was shown for example in Ref. [149]. A better approach for obtaining an integrated form of the surface energy, which is not restricted to cases where $\omega=0$, is to use Eq. (42). Combining this with expressions such as Eq. (47) to evaluate the adsorption terms for solute and solvent, the surface energy can readily be calculated from the surface composition information obtained by the model. The results are displayed in Fig. 20 for the surface composition data obtained in the case of a positive regular solution constant in Fig. 19a.

The slopes in the curves shown in Fig. 20 are related to the adsorption (see Eq. P4(2)41). As the bulk concentration of the solute, $x$, increases, at any given temperature, so does the adsorption (by Eq. 44), hence the gradual increase in absolute slope of the curves with increasing $x$. But note that the slope of the curves approaches an asymptotic value. This corresponds to saturation of the surface monolayer in solute. Note also, that although the surface becomes saturated in solute, the surface energy continues to decline. This points to a common misconception. The surface energy is not proportional to the adsorption, rather it is the slope, $\mathrm{d} \gamma / \mathrm{d} \mu_{\mathrm{B}}$, that is proportional to adsorption.

Finally, it is useful to cross-plot the results of Fig. 20, as $\gamma$ versus $T$ for constant bulk composition, as shown in Fig. 21. For low-bulk concentrations of solute, the surface energy increases with temperature but eventually reaches a plateau value corresponding to the surface energy of the pure solvent when $\Gamma_{\mathrm{B}}$ and its derivative are so small that there is longer any effect on the change in surface energy.

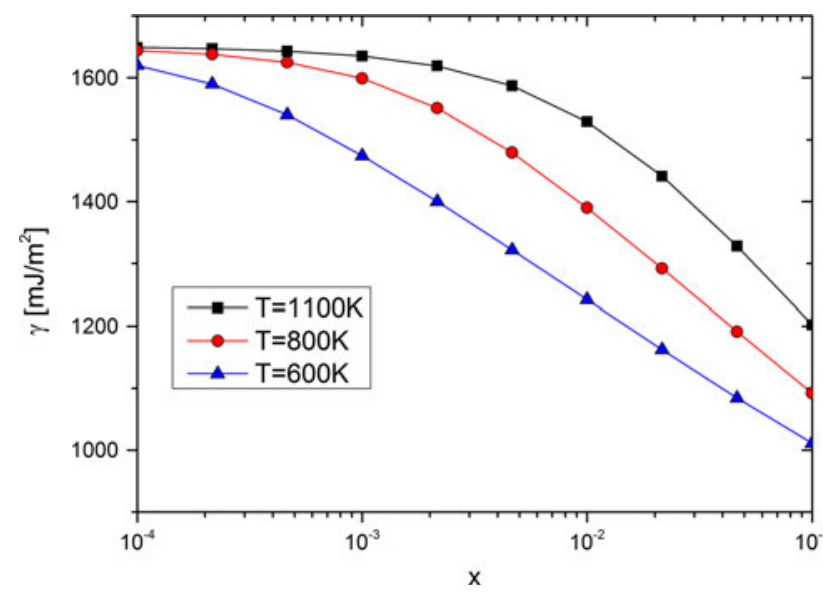

Fig. 20 Variation of the interfacial energy with bulk composition (log scale), at several temperatures, for values of the parameters used in the case $\omega=550 \mathrm{~J} / \mathrm{mol}$ in Fig. 16. $x$ is the atomic fraction of the solute component $\mathrm{B}$

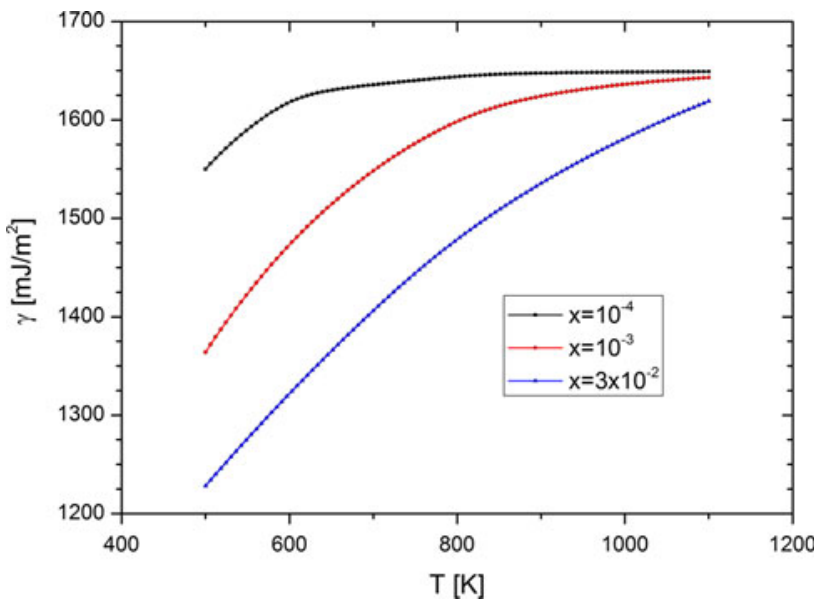

Fig. 21 Variation of surface energy with temperature for various values of the bulk solute concentration, cross-plotted from Fig. 17

More realistically, this low-bulk concentration curve should display a slightly negative slope above approximately $800 \mathrm{~K}$, because the surface energy of pure materials shows a small but finite decrease with increasing temperature. However, the main point illustrated by Fig. 21 is the strong temperature dependence of surface energy that can result from segregation. This is most evident at higher bulk concentrations of solute and results from the exponential decrease in segregation with increasing temperature illustrated in Fig. 19.

Although the trends illustrated in Figs. 19, 20, and 21 are for the case of surface segregation in a solid solution, they provide a useful qualitative picture of the effects of segregation on the energy of all interfaces.

\section{Effects of adsorption/segregation on wetting}

Wetting systems can be quite complex, thus for the sake of simplicity, we will consider a system consisting of two surfaces (those belonging to the liquid phase and to the solid substrate) and one solid/liquid interface. Both the wetting phase and the substrate may contain impurities and/or deliberate alloying additions, and these components can all segregate to some or all of the interfaces (two surfaces and one solid/liquid interface). In addition, any interfacially active species present in the environment (gas phase) surrounding the wetting system can also adsorb at these interfaces (see the effect of oxygen in [150-152]). Thus, in general, it is possible for the energies of any or all of the interfaces relevant to a wetting system to be modified by adsorption/segregation effects.

When adsorption is limited to a single interface, at such chemical potential(s) of the adsorbate(s) that its energy is decreased, the consequences on wetting are predictable on the basis of the Gibbs adsorption isotherm. Consider, for 
example, a case where (the contact angle) $\theta<90^{\circ}$, in which adsorption occurs exclusively at the surface of the wetting phase and decreases its energy, $\gamma_{\mathrm{LV}}$. This leads to a consequent decrease in contact angle, or an improvement in wetting. However, if $\theta>90^{\circ}$, a decrease of the surface energy of the wetting phase will increase the contact angle, leading to poorer wetting. Energy decrease related to adsorption confined to either of the other two interfaces (surface of the substrate or interface between the substrate and the wetting phase) does not produce this reversal of behavior at $\theta=90^{\circ}$. Rather, a decrease in $\gamma_{\mathrm{SV}}$ always produces poorer wetting, whereas a decrease in $\gamma_{\mathrm{SL}}$ always improves wetting.

When more than one of the interfaces that control wetting behavior decreases in energy because of adsorption (i.e., an increase of one or several $\Gamma_{i} \mathrm{~d} \mu_{i}$ ), the net effect can act either to increase or decrease the contact angle, and more detailed information about segregation to the various interfaces is needed before experimentally observed trends can properly be interpreted [153].

\section{Wetting transitions}

The existence of transitions from non-zero to zero contact angle, i.e., wetting transitions, were first proposed by Cahn [154] and first observed by Moldover and Cahn [155]. For the sake of simplicity, let us first consider two fluid phases $\left(\alpha^{\prime}\right.$ and $\left.\alpha^{\prime \prime}\right)$ in contact and at equilibrium with a solid substrate (s) such that Young's equation may be applied: $\gamma_{\alpha^{\prime} \alpha^{\prime \prime}} \cos \theta=\gamma_{s \alpha^{\prime \prime}}-\gamma_{s \alpha^{\prime}}$

The $\alpha^{\prime}$ and $\alpha^{\prime \prime}$ phases are taken to be in equilibrium below their critical point $\left(T_{\mathrm{c}}\right)$. These phases may consist of a liquid and its vapor, or of two partially miscible liquid phases. In either case, as $T_{\mathrm{c}}$ is approached from lower temperatures, the difference between the $\alpha^{\prime}$ and $\alpha^{\prime \prime}$ phases disappears and $\left(\gamma_{s \alpha^{\prime \prime}}-\gamma_{s \alpha^{\prime}}\right)$ will approach 0 as $\left(T_{\mathrm{c}}-T\right)^{1.3}$. Also, as $T_{\mathrm{c}}$ is approached from below, the interfacial energy $\gamma_{\alpha^{\prime} \alpha^{\prime \prime}}$ will vanish as $\left(T_{\mathrm{c}}-T\right)^{\beta}$, where $\beta \approx 0.3-0.4$. As a result, $\cos \theta$ will approach unity (i.e., $\theta \rightarrow 0$ ) at some positive value of $\left(T_{\mathrm{c}}-T\right)$. The value of $T$ corresponding to $\theta=0$ is referred to as the wetting temperature and will be denoted as $T_{\mathrm{W}}$.

From a materials perspective, it is convenient to discuss this type of transition in the context of a two-component system where two liquid phases can co-exist over a range of temperature and composition, within a miscibility gap, as illustrated in Fig. 22a. Now consider a system consisting of two liquid phases, $\alpha^{\prime}$ and $\alpha^{\prime \prime}$ and their vapor, $V$. In general, one of the two liquid phases (say $\alpha^{\prime \prime}$ ) will float at the surface of the other. At temperatures below $T_{\mathrm{W}}$, the configuration of the floating drop is as depicted in Fig. 22b, and displays a finite dihedral angle $\left(\theta_{1}>0, \theta_{2}>0\right)$; whereas at $T_{\mathrm{W}}, \theta_{1}+\theta_{2}=0$, with the result that $\alpha^{\prime \prime}$ spreads over the surface of $\alpha^{\prime}$ to form a wetting layer, as shown in Fig. 22c.

In the case of Fig. 22b, the interfacial equilibrium condition is given by Eq. (8) and may be written as:
Fig. 22 a Phase diagram plotted as $T$ versus the atom fraction of component $\mathrm{B}, x_{\mathrm{B}}$, showing a liquid-liquid miscibility gap and $T_{\mathrm{W}}$; b partial wetting configuration of the $\alpha^{\prime}-\alpha^{\prime \prime}$ - vapor system at $T<T_{\mathrm{W}}$; c wetting configuration of the $\alpha^{\prime}-\alpha^{\prime \prime}-$ vapor system at $T \geq T_{\mathrm{W}}$ (a)

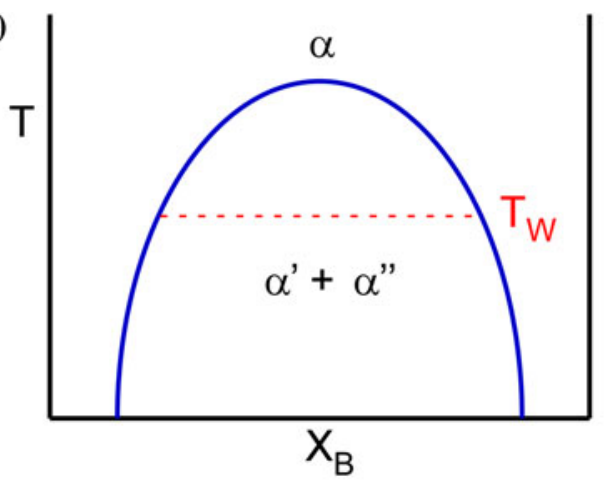

(b)

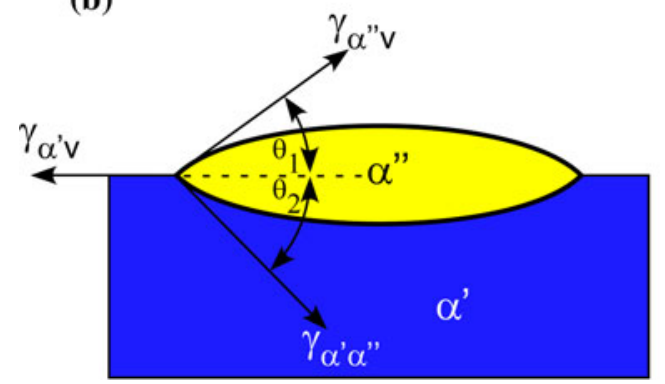

(c)

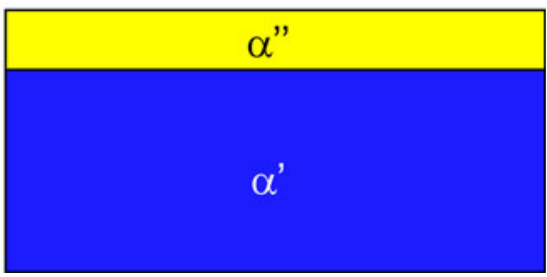


$\gamma_{\alpha^{\prime} V}=\gamma_{\alpha^{\prime} \alpha^{\prime \prime}} \cos \left(\theta_{2}\right)+\gamma_{\alpha^{\prime \prime} V} \cos \left(\theta_{1}\right)$

whereas in Fig. 22c, equilibrium is defined by:

$\gamma_{\alpha^{\prime} V}=\gamma_{\alpha^{\prime} \alpha^{\prime \prime}}+\gamma_{\alpha^{\prime \prime} V}$

Wetting transitions can be classified as being of first order or critical [156]. In both cases $\cos (\theta)$ approaches unity continuously as temperature is increased. However, its derivative with respect to $T$ can be either discontinuous at $T_{\mathrm{W}}$ (first-order wetting transition) or continuous (critical wetting transition). A variety of adsorption phenomena can occur in conjunction with wetting transitions, as described in the following section.

\section{Associated adsorption transitions}

Adsorption phenomena associated with wetting transitions have been reviewed by Schick [156] for the case of a gas adsorbing on a solid substrate; here we discuss this type of behavior in the context of a binary liquid system, of more interest to the high temperature wetting community.

The adsorption behavior is summarized in Fig. 23 for the case of a first-order wetting transition. Figure 23a reproduces part of the phase diagram shown earlier in Fig. 22a. In Fig. 23a, the locus of adsorption transitions or prewetting lines associated with the wetting transition are identified by dashed lines which lie to the left of the $\alpha^{\prime}-\alpha^{\prime \prime}$ coexistence line; in addition, three paths of approach to the coexistence line are indicated by horizontal arrows.

Figure $23 \mathrm{~b}$ shows the adsorption of component $\mathrm{B}$ at the surface of the $\alpha$ phase, as a function of the chemical potential of $\mathrm{B}, \mu_{\mathrm{B}}$. The vertical dashed line is drawn at $\mu_{0}$, the chemical potential of $\mathrm{B}$ at the edge of the miscibility gap, i.e., at $\alpha^{\prime}-\alpha^{\prime \prime}$ coexistence.

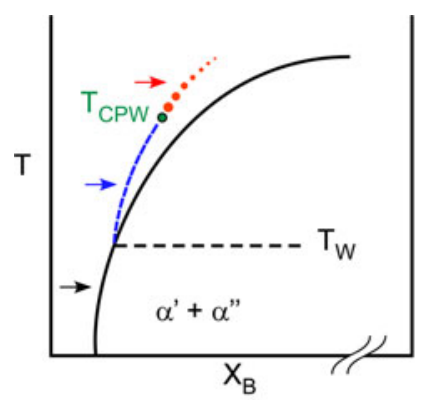

(a)

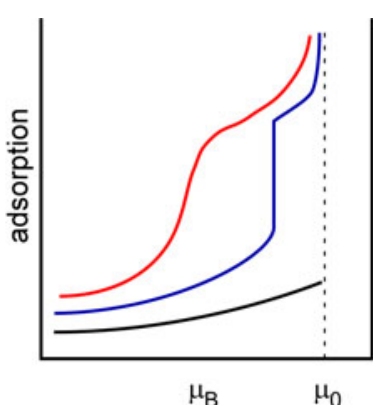

(b)
Fig. 23 a Part of the phase diagram shown in Fig. 22a. The dashed blue line designates the locus of prewetting adsorption transitions, which terminate at high temperature at the prewetting critical point ( $\left.T_{\mathrm{CPW}}\right)$, and the red dashed line shows the extension of the prewetting line above $T_{\mathrm{CPW}}$. In addition, three paths of approach to the edge of the miscibility gap are designated by arrows. b The adsorption behavior along the three paths identified in $\mathbf{a}$ is shown, as a function of the chemical potential (see text) (Color figure online)
Consider first the trajectory corresponding to the black arrow in Fig. 23a, along which coexistence is approached below $T_{\mathrm{W}}$. The corresponding black curve in Fig. 23b shows that in that case adsorption of B at the surface of $\alpha$ increases up to a finite value as coexistence is reached. Indeed, the presence of finite adsorption at coexistence is evidence that when the $\alpha^{\prime \prime}$ phase forms at the surface of $\alpha^{\prime}$, it only partially wets the $\alpha^{\prime \prime}$ phase.

Now consider the trajectory corresponding to the blue arrow in Fig. 23a, which lies above $T_{\mathrm{W}}$. As this path crosses the dashed blue prewetting line, B-adsorption at the surface of the $\alpha$ phase undergoes a first-order jump, as shown in the blue line of Fig. 23b (we recall that the surface energy at this transition only changes slope as described by the Gibbs adsorption equation (Eqs. 35, 38). As the path continues towards the two-phase coexistence line, the adsorption diverges, presaging the formation of the bulk $\alpha^{\prime \prime}$ phase at coexistence. When coexistence is reached, the newly formed $\alpha^{\prime \prime}$ phase completely wets the surface of the $\alpha^{\prime}$ phase, since the temperature lies above $T_{\mathrm{W}}$.

For other trajectories that cut the prewetting line, between its lower end at $T_{\mathrm{W}}$ on the edge of the miscibility gap and its upper end at the prewetting critical point $\left(T_{\mathrm{CPW}}\right)$, the magnitude of the first-order jump in B-adsorption gradually diminishes and eventually vanishes at $T_{\mathrm{CPW}}$. Above $T_{\mathrm{CPW}}$, along trajectories corresponding to the red arrow in Fig. 23a, higher order adsorption transitions occur as the extension of the prewetting line (dashed red line) is crossed, and adsorption continues to diverge in the limit of coexistence, as shown by the red line in Fig. 23b. Here also, complete wetting is achieved at coexistence.

It is also useful to consider a trajectory that follows the edge of the miscibility gap approaching $T_{\mathrm{W}}$ from below. In this case, adsorption is finite below $T_{\mathrm{W}}$, and jumps discontinuously to infinity at $T_{\mathrm{W}}$, in accordance with expectations for a first-order transition. In all of the preceding discussion, one should recognize that when the coexistence line is approached from the single-phase domain, divergence of the adsorption indicates the formation of the bulk $\alpha^{\prime \prime}$ wetting phase at the surface of the $\alpha^{\prime}$ phase, whereas finite adsorption is the conventional adsorption/segregation phenomenon that prevails in the absence of complete wetting.

In addition to the types of transitions described in Fig. 23, Pandit et al. [157] have developed a model in which layered adsorption transitions occur when certain relationships between adsorbate-adsorbate and adsorbatesubstrate interactions prevail. In layered transitions, the region containing the adsorbed species increases in thickness, one atomic layer at a time, by a series of either first or higher order transitions. In contrast to Cahn's, model, these 
transitions can occur even below the wetting temperature, as shown schematically in Fig. 24. Figure 24a shows a portion of the phase diagram shown previously in Fig. 22a, with dashed lines representing adsorption transitions that occur in the single $\alpha$-phase domain. Each dashed line represents a first-order adsorption transition in a single atomic layer. Figure 24b displays the adsorption as a function of chemical potential (or increasing bulk composition) of the adsorbing species, B. Consider the changes in adsorption shown by the blue curve in Fig. 24b, which occur along the path indicated by the blue arrow in Fig. $24 \mathrm{a}$, at a temperature below $T_{\mathrm{W}}$. As $x_{\mathrm{B}}$ (or $\mu_{\mathrm{B}}$ ) increases, adsorption at the surface of the $\alpha$-phase increases monotonically until it crosses the first dashed line (terminating in a critical point at $\left.T_{\mathrm{c} 1}\right)$. Where it crosses the dashed line, a first-order transition in adsorption will occur in the first atomic layer of the surface. As the chemical potential continues to increase, the second dashed line (terminating in a critical point at $T_{\mathrm{c} 2}$ ) is crossed, and this corresponds to a first-order transition in the second surface layer, as shown in the blue curve of Fig. 24b. In principle, the number of layers that can undergo transitions is not limited, but must be finite as long as the path followed lies below $T_{\mathrm{W}}$. Also, the thickness of the layers which undergo transition may not be limited to one atomic layer, but depend on the range of the interatomic interactions.

Paths at temperatures above $T_{\mathrm{W}}$ differ in that the number of layers undergoing transitions is unlimited, and the total adsorption will diverge, as was the case for the transitions shown in Fig. 23. If the path is as indicated by the red arrow, where all the dashed lines are crossed below their respective critical temperatures, there will be an infinite number of atomic layers undergoing first-order transitions (as in the red curve shown in Fig. 24b). Conversely, along

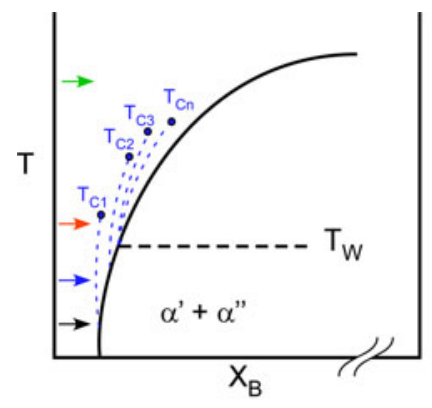

(a)

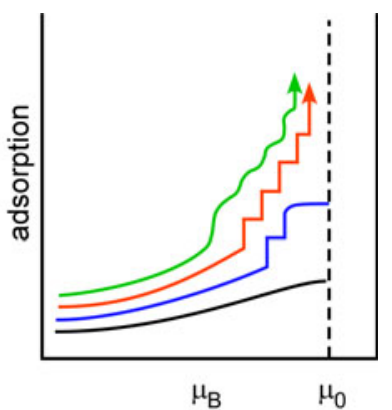

(b)
Fig. 24 a Part of the phase diagram shown in Fig. 19a. The dashed blue lines designate the locus of adsorption transitions, each of which terminates at high temperature at a critical point $\left(T_{\mathrm{Ci}}\right)$. In addition, four paths of approach to the edge of the miscibility gap are designated by arrows. b The adsorption behavior along the four paths identified in $\mathbf{a}$ is shown, as a function of the chemical potential, as in Fig. 20 (Color figure online) the green path, which lies above all of the layer critical temperatures, a series of higher order adsorption transitions will occur in each atomic layer. Intermediate paths between the red and green paths will display some high order and some first-order transitions.

Thus far, we have focused on first-order wetting transitions. As mentioned earlier, wetting transitions can also be critical. In this case, if one follows a trajectory along the edge of the miscibility gap towards $T_{\mathrm{W}}$ from lower temperatures, adsorption will increase continuously and diverge as $T_{\mathrm{W}}$ is approached. In the case of critical wetting transitions there are no associated adsorption transitions such as the prewetting phenomena described above; isothermal paths toward coexistence result either in finite adsorption at coexistence, if $T<T_{\mathrm{W}}$ (as in the black curve of Fig. 24b), or in continuously increasing adsorption with divergence at coexistence, for paths that approach coexistence at $T>T_{\mathrm{W}}$.

Jumps in adsorption do not mean jumps in surface energy

Whereas phase transitions in bulk phases at the equilibrium transition temperature (e.g., the transition from solid to liquid at the melting point) are universally understood to occur when the free energies of the two phases are equal, there is some confusion on this point where adsorption transitions are concerned. When two bulk phases coexist in equilibrium, the chemical potentials of each of the components in the phases must be equal, and this is also true for coexisting adsorption states. This is illustrated in Fig. 25, which has been generated by means of a multi-layer model similar to that described in section "Illustration of adsorption/segregation by means of a simple model", using parameters that give rise to a series of first-order adsorption transitions [158].

Figure 25a is a plot of $\mathrm{Pb}$ adsorption versus the bulk atom fraction of $\mathrm{Pb}, x$, at the surface of a liquid $\mathrm{Ga}-\mathrm{Pb}$ alloy, at a temperature of $500 \mathrm{~K}$. Here the surface of the liquid has been modeled as the (111) surface of a fcc solid [158]. The figure shows an adsorption transition in the first atomic layer at the surface. This temperature is above $T_{\mathrm{W}}$ for the alloy. Although the alloy does display an infinite number of atom layer transitions, only the first of these is shown in the figure, as the second and subsequent layers undergo transitions at values of $x$ that lie between the highest value plotted $(x=0.0079)$ and the phase boundary $\left(x_{0} \sim 0.0092\right)$ indicated on figure by an arrow. Even the second layer transition would be difficult to display as it occurs at $x_{0}-x \sim 10^{-4}$ [158]. The solid line in Fig. 25b gives the surface energy variation with $\mathrm{Pb}$ bulk atom fraction corresponding to Fig. 25a. It clearly shows that the surface energy at the bulk composition corresponding to 

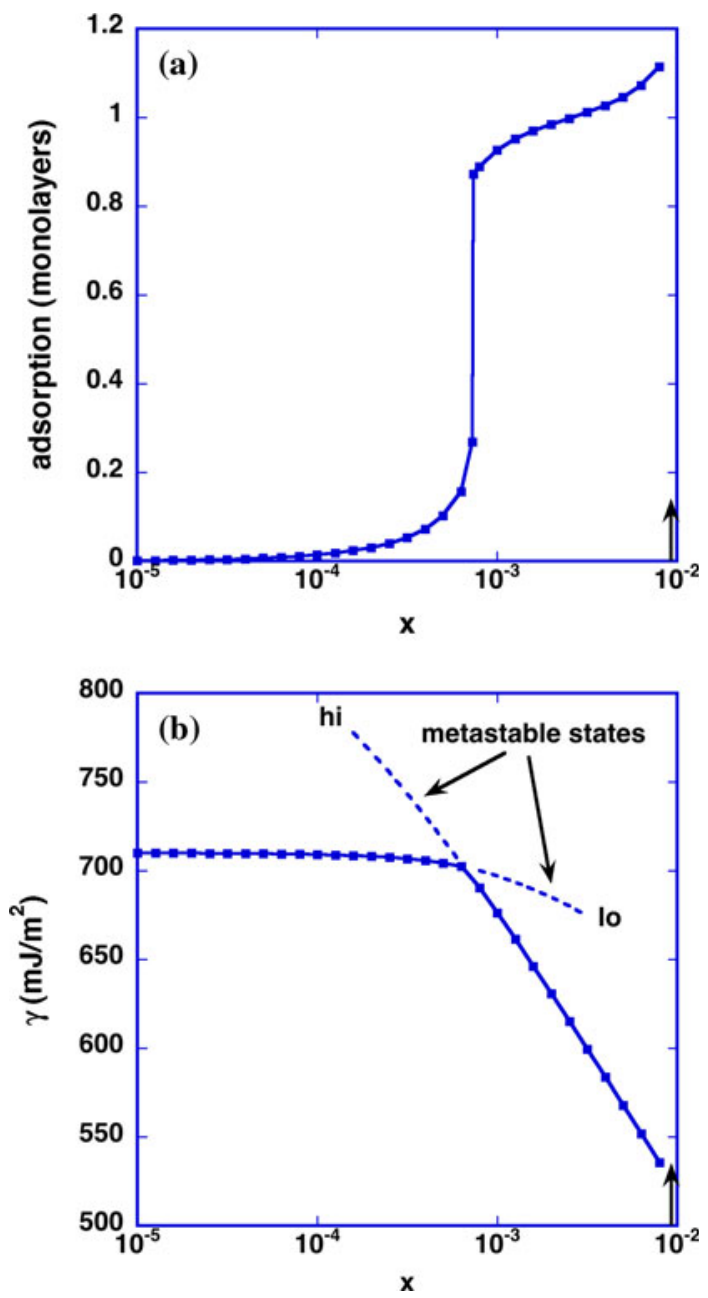

Fig. 25 a Plot of adsorption at the surface of a liquid $\mathrm{Ga}-\mathrm{Pb}$ alloy versus $\mathrm{Pb}$ bulk composition, showing an adsorption transition in the first surface layer. b Corresponding plot of surface energy; the solid line shows the locus of equilibrium states and displays a discontinuity in slope at the composition of the transition, but equal surface energies for both the high and low adsorption states; the dashed lines indicate the metastable high and low adsorption states. The arrows mark the value of $x$ corresponding to the phase boundary

the transition is discontinuous, but that the values of the surface energies that correspond to the coexisting low and high adsorption states are identical. The dashed lines in Fig. 25b are schematic, and illustrate the approximate surface energies of metastable states that could exist for the high and low adsorption states above and below the transition temperature.

What does "monolayer" adsorption mean?

The term monolayer adsorption/segregation is ambiguous in many situations. Here we consider several possible cases in order to illustrate this issue.

Consider first a case of adsorption from the gas phase onto an elemental crystalline solid surface, as depicted schematically in Fig. 17b. For the case of a close packed solid surface, it might be reasonable to assume that the number of adsorption sites in the layer above the outermost crystal layer is equal to the number of atomic sites in the crystalline layer. If all adsorption sites were occupied by adsorbate atoms, then one could describe this state as one depicting monolayer adsorption. For the sake of simplicity, let us consider that the adsorbate species behaves as hard spheres, and that the atomic radius of the adsorbate atoms is larger than that of the substrate atoms. Under these conditions, as the adsorption sites of the substrate surface become progressively occupied, a saturation point will be reached where no more adsorbate atoms can be accommodated, leading to a state where only a fraction of the adsorption sites, $f<1$, are filled. Conversely, for an adsorbate with an atomic radius smaller than that of the substrate atoms, saturation would occur for $f>1$. In both of these cases, however, the surface could be viewed as consisting of a "monolayer" of adsorbate. This is clearly ambiguous. But the situation is actually much more complicated than discussed thus far. What if the crystalline substrate surface consisted of some high index plane, e.g., (321) of a fcc solid where several (321) near-surface atomic layers lack complete coordination? Now, even the number of adsorption sites becomes difficult to define, as the adsorption sites lie on different atomic layers of the substrate.

In the case of segregation from the bulk, such as depicted schematically in Fig. $17 \mathrm{~d}$, new complications arise in defining the state of the system in terms of monolayers. This is due to the fact that adsorption generally occurs in several near-surface atomic layers of the substrate, and that each layer consists of a mixture (solution) of both components. Thus, when the topmost surface layer is close to saturation, there will also be non-negligible adsorption occurring in deeper layers. This situation was also illustrated in Fig. 19b, which shows that even though the uppermost atomic layer (layer 1) is essentially saturated with the segregating component, non-negligible segregation also occurs in layers 2 and 3.

Some of the issues alluded to here can be avoided by employing a Gibbsian definition of adsorption. Then, adsorption is defined as the sum of the number of adsorbate species present in the near-surface region that exceed (positively or negatively) their number in an equivalent region of the bulk phase, expressed per unit area of surface. With this approach, the term "monolayer" adsorption ceases to be meaningful, unless one arbitrarily defines a certain number of adsorbate species per unit area as constituting a monolayer. Even this approach suffers from the disadvantage that the distribution of adsorbate species in the near-surface region is not evident. Nevertheless, this discussion should make it clear that the terminology of monolayer adsorption/segregation is best avoided. 


\section{Complexions}

What are complexions and complexion transitions?

The discussion in the previous sections clearly shows that a first (or higher) order transition can occur in the state of an interface, surface, or grain boundary, with regards to chemical excess (adsorbate). Recently, these transitions have been shown to have a significant influence on the properties of grain boundaries with regards to mobility during grain growth [159]. Scanning TEM of similar grain boundaries in the same samples suggested that the changes in grain boundary mobility are dependent upon the state of the grain boundaries with regards to transitions in adsorption, which were classified by Dillon et al. [160] as specific states according to their structural width (rather than chemical content). A structural width is a useful characterization; see comments in Eq. (59).

The concept of structural (rather than chemical) changes at a grain boundary is not new, but the recent models by Tang et al. proposed that structural transitions could be seen as first-order transitions, in a manner analogous to the adsorption transitions discussed in section "Microscopic scale and adsorption" [161-163]. Unfortunately these transitions were described by some as phase transitions, or grain boundary phase transitions, while the transitions in adsorption or structure were clearly confined to the $2 \mathrm{D}$ state of the interface. As a result, Cannon and Carter suggested calling this general phenomenon "complexion" transitions, where a complexion is an equilibrium 2D state of an interface [161]. It is important to differentiate between bulk phases and complexions, since complexions can only exist at the surface of a bulk phase or at the interface between two bulk condensed phases, and complexion transitions can occur without a transition in any of the adjacent bulk phases.

Interestingly, structural transitions at an interface, without any nominal change in chemistry (chemical excess) have recently been studied during wetting experiments of liquid $\mathrm{Al}$ in contact with sapphire $[132,164]$. The ordered region in the liquid adjacent to the solid is, in effect, a complexion, where the change in order as a function of, e.g., temperature or terminating solid facet plane, are different complexions [165]. That this phenomenon is a complexion is inferred from the experimental data, but cannot be directly proven. However, recently the energy of $\mathrm{Au}-$ sapphire interfaces with and without a $\mathrm{Ca}-\mathrm{Si}$-based complexion were measured, and a significant difference in energy was experimentally associated with the change in the interface complexion and an increase of the chemical potentials of Si and Ca [166, 167].

An interface will have an equilibrium complexion at given values of system potentials such as pressure, temperature, and the chemical potentials of the various chemical species. For crystalline surfaces, the equilibrium complexion will depend on the surface orientation; for example, in one-component systems, the surface can undergo reconstruction, disorder, roughening or melting [168]. In binary alloys, several surface complexions have been observed, which differ in structure and composition depending on the chemical potentials [169-172]. Symmetry reconstructions at the surfaces of oxides have been studied by Rappe and co-workers [173] who plotted the equilibrium surface structures as a function of the potentials (e.g., the partial pressure of oxygen). From this discussion it is clear that surface reconstruction is necessarily a complexion transition.

At grain boundaries, complexions manifest themselves as highly localized structural changes, or as abrupt changes in the adsorption content, which display a width set by thermodynamic equilibrium, and whose presence can lead to changes in properties such as electrical conductivity, adhesion, and grain boundary mobility [63, 174-179]. In all cases, these phenomena can be treated with an identical and rigorous classical thermodynamic foundation [163]. These formulations are not new. Gibbs recognized that soap film transitions can be treated in the same manner as the equilibrium of heterogeneous materials [180]; Smith [181] provided a heuristic argument for the onset of a premelting film of finite thickness; Cahn [154] extended this idea to compositional changes in his diffuse interface treatment in critical wetting theory; Schick [156] extended this further by a careful thermodynamic treatment of the interface environment. Abrupt surface symmetry changes, and surface relaxation are also well known. Several phenomena were described in Cahn's [154] critical wetting paper, including the abrupt transition of surface adsorption, $\Gamma$, at the exterior surface of a single phase. The term "critical wetting" has been used to describe these abrupt changes in adsorption, and this is unfortunate because these interfacial structures and compositions are not "wetting" and should not be directly associated with a wetting angle.

There is a tendency to call complexion transitions "phase transitions" and the finite films that constitute the complexions have been called "phases." This is also unfortunate. Complexions are not phases as defined by Gibbs (p. 96 in Collected Works [180]) “...the thermodynamic state of any such body without regard to its quantity or form. ...Phases (are in equilibrium) when (they) can exist together, the dividing surfaces being plane, in an equilibrium that does not depend on passive resistance". Complexions cannot exist as a bulk phase. Their compositions and structures do not necessarily appear on an equilibrium phase diagram, and consideration of these structures as phases could lead to mistaken additional degrees of freedom, and thus misinterpretations of the Gibbs phase rule. Thus, these collective phenomena have been called "complexions" 
with the objective of associating them with interfaces exclusively, and remove direct association to phases.

Complexion transitions can manifest themselves in conjunction with discontinuous changes in material behavior. Dillon et al. [160] have identified six different grain boundary structures in alumina that correlate directly with grain boundary mobility. Given the number of degrees of freedom of the various grain boundaries in a polycrystalline alumina, it is unlikely that the number of complexions is limited to six. However, it may be that, at high temperatures, a relatively small number of grain boundary structures emerge and dominate behavior. Luo and co-workers [182] have associated activated sintering with complexions, and it would be expected that creep behavior, etc., will also depend on complexions. Crystals with many atoms per unit cell such as oxides, especially those which have tightly bound subunit cell structural units, such as silica tetrahedral, are likely candidates for disordered complexions.

Methods of treating interfaces with $C$ components and $\Phi$ phases and its connection to complexions

Cahn [23] provides a useful and rigorous method of treating the excess quantities associated with an interface in a multiphase multi-component system. This section provides a trivial extension of Cahn's method to multiple interfaces. Recently Frolov and Mishin [183] have used this method to include the effect of mechanical stresses on the interfacial region.

The thermodynamic system consists of reservoirs of each of the $C$ independent chemical species; these reservoirs maintain constant chemical potentials, but their values may be varied (e.g., $\mathrm{d} \mu_{\mathrm{i}}$ ) by an experimenter. An additional reservoir of an inert species allows the total pressure (i.e., the sum of partial pressures) to be controlled independently. The entire system has a thermally conductive barrier in contact with a thermal reservoir which maintains a constant temperature. The experimenter has $C+2$ knobs that can be adjusted independently. The pressures within each phase must be uniform, but can take on different values within isolated bodies according to the value of $\gamma \kappa$ for isotropic surfaces (where $\kappa$ is the curvature of the interface) or weighted mean curvature $\kappa_{\gamma}$ [50] for anisotropic surfaces; equilibrium requires that these quantities be uniform and have the same value on all surfaces which bound an isolated body. The system is treated as a restricted equilibrium wherein mass exchange across the interfaces is slow compared to all other processes (i.e., grain growth is slow), and this is artificial because redistribution of the species transported from the chemical reservoirs is permitted. There are other systems which might be considered. For example, if the system is closed so that the number of each species is fixed, then the Gibbs free energy would be used. Here, the grand canonical potential, $\Omega$, is used in what follows. The Gibbs free energy function would be appropriate for nanocrystalline systems in which behavior is determined by redistribution of the adsorbate species between the bulk and interfacial regions.

Each phase has equilibrium densities associated with it, each of which depends on the various fixed and uniform potentials, the values of each phase's densities change according to a Gibbs-Duhem equation for each phase. The discussion is simplified by gravimetric densities (i.e., the number of species in a phase per unit mass, the phase volume per unit mass, and the phase entropy per unit mass). Volumetric densities would require the introduction of a reference volume.

Values of each phase's densities change according to a Gibbs-Duhem equation for each of the $\Phi$ phases, yielding $\Phi$ equations with $C+2$ unknowns.

$$
\begin{array}{ll}
\text { vap-phase } & 0=-s^{\mathrm{vap}} \mathrm{d} T+v^{\mathrm{vap}} \mathrm{d} P-\sum n_{i}^{\mathrm{vap}} \mathrm{d} \mu_{i} \\
\text { liq-phase } & 0=-s^{\mathrm{liq}} \mathrm{d} T+v^{\mathrm{liq}} \mathrm{d} P-\sum n_{i}^{\mathrm{liq}} \mathrm{d} \mu_{i} \\
\alpha \text {-phase } & 0=-s^{\alpha} \mathrm{d} T+v^{\alpha} \mathrm{d} P-\sum n_{i}^{\alpha} \mathrm{d} \mu_{i} \\
\vdots & \vdots \\
\pi \text {-phase } & 0=-s^{\pi} \mathrm{d} T+v^{\pi} \mathrm{d} P-\sum n_{i}^{\pi} \mathrm{d} \mu_{i}
\end{array}
$$

These $\Phi$ equations with $C+2$ unknowns result in $\mathrm{DoF}$ degrees of freedom (i.e., the maximum possible number of coexistent phases: $\mathrm{DoF}=C+2-\Phi)$ in the Gibbs phase rule.

Each of the interfaces has a relationship between the change in interfacial energy and its interfacial densities (i.e., $[S],[V],\left[N_{i}\right]$ where [] indicates division by a fixed reference area).

Interface Relations: $Q$ equations and $Q$ additional unknowns

$$
\begin{array}{ll}
\text { Liquid|Vapor: } & \mathrm{d} \gamma^{1 \mid \mathrm{v}}=-[S]^{1 / \mathrm{v}} \mathrm{d} T+[V]^{1 \mid \mathrm{v}} \mathrm{d} P-\sum\left[N_{i}\right]^{1 \mid \mathrm{v}} \mathrm{d} \mu_{i} \\
\text { Liquid } \mid \alpha: & \hat{n}^{\alpha} \cdot \mathrm{d} \vec{\xi}=-[S]^{1 \mid \alpha} \mathrm{d} T+[V]^{1 \mid \alpha} \mathrm{d} P-\sum\left[N_{i}\right]^{1 \mid \alpha} \mathrm{d} \mu_{i} \\
\alpha\left(0_{x}^{\alpha}\right) \mid \alpha\left(0_{y}^{\alpha}\right): & \hat{n}_{x}^{0_{x}^{\alpha} \mid 0_{y}^{\alpha}} \cdot \mathrm{d} \vec{\xi}=-[S]^{0_{x}^{\alpha} \mid 0_{y}^{\alpha}} \mathrm{d} T+[V]^{0_{x}^{\alpha} \mid 0_{y}^{\alpha}} \mathrm{d} P-\sum\left[N_{i}\right]^{0_{x}^{\alpha} \mid 0_{y}^{\alpha}} \mathrm{d} \mu_{i} \\
\vdots & \vdots \\
\beta\left(0_{x}^{\beta}\right) \mid \alpha\left(0_{y}^{\beta}\right): & \hat{n}_{x}^{\alpha} \mid 0_{y}^{\beta} \cdot \mathrm{d} \vec{\xi}=-[S]^{0_{x}^{\alpha} \mid 0_{y}^{\beta}} \mathrm{d} T+[V]^{0_{x}^{\alpha} \mid 0_{y}^{\beta}} \mathrm{d} P-\sum\left[N_{i}\right]^{0_{x}^{\alpha} \mid 0_{y}^{\beta}} \mathrm{d} \mu_{i}
\end{array}
$$


The system gains no additional degrees of freedom: DoF of the unknowns $\left(\mathrm{d} \mu_{i}, \mathrm{~d} T, \mathrm{~d} P, \mathrm{~d} \gamma\right.$, etc.) can be solved in terms of the remaining $C+2-\Phi$ unknowns. The extra $Q$ unknowns differ in that they are internal variables: the experimenter has no means to adjust these quantities individually, but only indirectly through $P, T$, and $\mu_{i}$.

The above notation is cumbersome but necessary: $\hat{n}^{\alpha}$ is the orientation of the crystalline $\alpha$-interface with respect to a reference frame. $\vec{\xi}$ is the Cahn-Hoffman anisotropic capillarity vector (which reduces to $\hat{n}^{\alpha} \gamma$ in the isotropic case). The Cahn-Hoffman $\vec{\xi}$ is discussed elsewhere [49], for the present purposes it suffices to recognize that $\hat{n}^{\alpha} \cdot \mathrm{d} \vec{\xi}$ represents the change in the interfacial energy for a crystalline orientation $\hat{n}^{\alpha} .0_{x}^{\beta} \mid 0_{y}^{\alpha}$ represents the interface between a $\beta$-phase (with a crystallographic orientation $x$ with a normal $\hat{n}^{\beta}$ ) and an $\alpha$-phase (with crystallographic orientation $y$ with a normal $\hat{n}^{\alpha}$ ). Using an outward normal convention, $\hat{n}^{\alpha}=-\hat{n}^{\beta}$, because both normals share the same reference frame.

Each interface must be in equilibrium with its abutting phases and examples are given above. However, because there may be extant equilibrium phases that do not abut a particular interface, the Gibbs-Duhem equation for that non-abutting phase must also appear as one of the equations used for elimination. Therefore, the behavior of an $\alpha-\alpha$ grain boundary at a range of compositions where only the $\alpha$ phase is in equilibrium will differ from an $\alpha-\alpha$ grain boundary when a $\beta$-phase is also at equilibrium. In the first case, the adsorption of a species (e.g., B) will change continuously until a complexion transition occurs, at which point the adsorption changes discontinuously at constant interfacial energy; upon further addition of B to the system, a wetting transition occurs when another phase (e.g., $\beta$ ) appears. In the latter case, partial or complete wetting of the grain boundary by the $\beta$-phase is always possible.

In addition to the Gibbs-Duhem equations for multicomponent and multiphase systems, one may choose to use an interface equation for each interface in Fig. 26. In this case, an adsorption isotherm equation will appear for each interface, but each will depend on the potentials that the experimentalist chooses to vary independently. In other words, changing the potentials in Fig. 26 will cause simultaneous changes on all the interfaces in a manner that is calculated above. These interfaces are not available to be controlled independently.

The coupling of the interface equations at junctions, such as triple lines, has not been included in the equations above. If they were to be included, then they would give

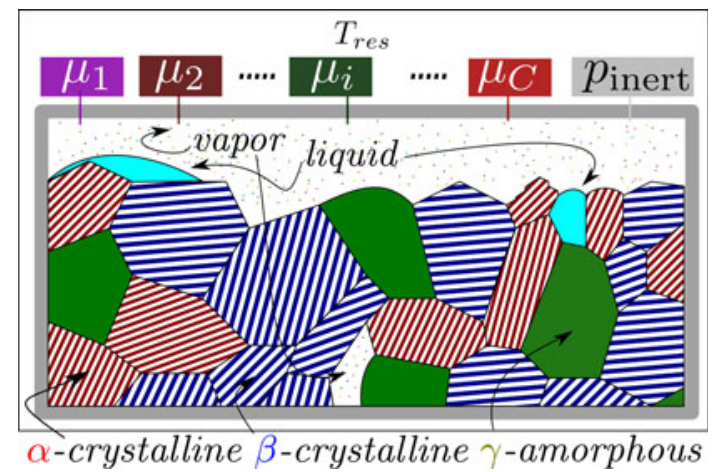

Fig. 26 A thermodynamic system with a fixed pressure, temperature, and chemical potentials of $C$ independent components. The experimentalist has the possibility of controlling any of these potentials, but the number of potentials that can be varied independently and maintain phase coexistence is determined by the Gibbs phase rule. The figure illustrates the variety of interfaces that can be considered: vapor/liquid, liquid/crystalline, vapor/crystalline, two-phase crystalline/crystalline, single-phase crystalline/crystalline, amorphous/crystalline, etc. This image is used to illustrate the development of the Gibbs adsorption isotherms for the general case of many interface types in multi-component, multiple alloy systems.

geometric boundary conditions (i.e., the contact angles) for the intersections and this discussion appears in Gibbs (Figs. 11-13 in p. 287 of [184]). Any additional energies such as triple line energies have not been included, but as Gibbs states (footnote on p. 288 of Collected Works [184]) the triple junctions can be treated analogously to interfaces, but with a line replacing a cylinder of localized inhomogeneity instead of a dividing surface replacing a localized slab of inhomogeneous material.

Connection to complexions and wetting transitions

A complexion is associated with particular values of $[S]^{0_{x}^{\alpha} \mid 0_{y}^{\beta}},[V]^{0_{x}^{\alpha} \mid 0_{y}^{\beta}}$, and $\left[N_{i}\right]^{0_{x}^{\alpha} \mid 0_{y}^{\beta}}$. (Note that $[V]^{0_{x}^{\alpha} \mid 0_{y}^{\beta}}$ has units of a characteristic width of the interface, which can be used to characterize a complexion transition.) A first-order complexion transition would be associated with finite changes of $[S]^{\alpha_{x}^{\alpha}}\left|0_{y}^{\beta},[V]^{0_{x}^{\alpha}}\right| 0_{y}^{\beta}$, and $\left[N_{i}\right]^{0_{x}^{\alpha} \mid 0_{y}^{\beta}}$. At particular values of $T, P$, and $\mu_{i}$ which define the edge of the miscibility gap, an additional phase is in equilibrium with $\alpha$ and $\beta$, and then DoF (i.e., DoF $=C+2-\Phi$ ) will decrease by 1 .

Each particular interface must be in equilibrium with its abutting phases, or phase in the case of a grain boundary. It is useful to consider several different cases that illustrate the differences in the computation of the adsorption or excess quantities; below we consider examples of grain boundaries in single-phase alloys, and interphase boundaries in multi-component alloys. 
Isotropic grain boundary $(\alpha / \alpha)$, unary phase (A)

There is one equation of the Gibbs-Duhem type and one interface equation. Eliminating $\mathrm{d} P$ :

$\mathrm{d} \gamma^{\mathrm{gb}}=\frac{n_{\mathrm{A}}^{\alpha}[V]^{\mathrm{gb}}-\left[N_{\mathrm{A}}\right]^{\mathrm{gb}} v_{\mathrm{A}}^{\alpha}}{v_{\mathrm{A}}^{\alpha}} \mathrm{d} \mu_{\mathrm{A}}+\frac{s^{\alpha}[V]^{\mathrm{gb}}-[S]^{\mathrm{gb}} v_{\mathrm{A}}^{\alpha}}{v_{\mathrm{A}}^{\alpha}} \mathrm{d} T$

If the method of the Gibbs' dividing surface is applied to the case of a grain boundary, then the position of the dividing surface is arbitrary. If that position is chosen so that $[V]^{\mathrm{gb}}=0$, then for the isothermal case, $\mathrm{d} \gamma^{\mathrm{gb}}=$ $-\left[N_{\mathrm{A}}\right]^{\mathrm{gb}} \mathrm{d} \mu_{\mathrm{A}} \equiv-\Gamma_{\mathrm{A}}^{\mathrm{gb}} \mathrm{d} \mu_{\mathrm{A}}$; this choice $\left([V]^{\mathrm{gb}}=0\right)$ used by Gibbs is natural if the interface is taken to be a mathematical surface, however, $[V]^{\mathrm{gb}}$ is perhaps the most useful characteristic of complexion transitions and a nonzero choice is important in their characterization. $\left[N_{\mathrm{A}}\right]^{\mathrm{gb}}$ is the excess number of atoms associated with the grain boundary, this quantity is generally negative (which means there is a depletion of atoms per unit area of grain boundary), but can be of either sign.

Note that the elimination of the variable $\mathrm{d} P$ produces an expression which specifies the necessary variation of $P$, in other words changes of $P$ are not independent of $\mathrm{d} \mu_{\mathrm{A}}$ and $\mathrm{d} T$. This will be the case for all eliminated variables below.

Anisotropic grain boundary $(\alpha / \alpha)$, binary alloy (A-B)

There is one equation of the Gibbs-Duhem type and one anisotropic interface equation: the $\mathrm{d} \gamma^{\mathrm{gb}}$ is replaced with $\hat{n}_{x}^{\alpha}||_{y}^{\alpha} \cdot \mathrm{d} \vec{\xi}$. This equation has additional degrees of freedom because the adsorption or the excess entropy will depend
Eliminating $\mathrm{d} P$ using the single Gibbs-Duhem equation

$$
\begin{aligned}
\left.\hat{n}^{0^{\alpha}}\right|_{y} ^{\alpha} \cdot \mathrm{d} \vec{\xi}= & \frac{n_{\mathrm{A}}^{\alpha}[V]^{\mathrm{gb}}-\left[N_{\mathrm{A}}\right]^{\mathrm{gb}} v_{\mathrm{A}}^{\alpha}}{v_{\mathrm{A}}^{\alpha}} d \mu_{\mathrm{A}} \\
& +\frac{n_{\mathrm{B}}^{\alpha}[V]^{\mathrm{gb}}-\left[N_{\mathrm{B}}\right]^{\mathrm{gb}} v_{\mathrm{B}}^{\alpha}}{v_{\mathrm{A}}^{\alpha}} \mathrm{d} \mu_{\mathrm{B}} \\
& +\frac{s^{\alpha}[V]^{\mathrm{gb}}-[S]^{\mathrm{gb}} v_{\mathrm{A}}^{\alpha}}{v_{\mathrm{A}}^{\alpha}} \mathrm{d} T
\end{aligned}
$$

In this case, there are two different Gibbs adsorption isotherm terms: one for the excess of the A-species and one for the B-species. In the example of a two-phase binary alloy given below, the loss of one degree of freedom will result in an equation with only one adsorption isotherm for the species $\mathrm{B}$.

The $\vec{\xi}(\hat{n})$ are directly related with the "grain boundary Wulff shape": for each $\hat{n}$, the $\vec{\xi}(\hat{n})$ is the vector which coincides with a point on the Wulff shape having that $\hat{n}$. Thus, any point that lies on the grain boundary Wulff shape consists of the set of $\vec{\xi}(\hat{n})$ such that $\hat{n}$ is a stable orientation on the Wulff shape. In the trivial case that the Wulff shape is a circle: $\vec{\xi}(\hat{n})=\gamma^{\mathrm{gb}} \hat{n}$.

Liquid-vapor (llv), binary alloy (A-B)

There are two equations of the Gibbs-Duhem type with $C=2$ (one for each phase), and one of the interface type. These two equations are used to solve for the changes in potentials that maintain equilibrium: Eliminating $\mathrm{d} P$ and $\mathrm{d} \mu_{\mathrm{A}}$ from the two Gibbs-Duhem relations, and inserting these into the expression for $\mathrm{d} \gamma^{\mathrm{ll}} \mathrm{v}$ :

$$
\begin{aligned}
& \mathrm{d} \gamma^{\mathrm{l} \mid \mathrm{v}}=\frac{\left[N_{\mathrm{B}}\right]^{1 \mathrm{v}}\left(n_{\mathrm{A}}^{\text {vap }} v^{\text {liq }}-n_{\mathrm{A}}^{\text {liq }} v^{\text {vap }}\right)-\left[N_{\mathrm{A}}\right]^{1 \mathrm{v}}\left(n_{\mathrm{B}}^{\text {vap }} v^{\text {liq }}-n_{\mathrm{B}}^{\text {liq }} v^{\text {vap }}\right)+[V]^{1 / \mathrm{v}}\left(n_{\mathrm{B}}^{\text {vap }} n_{\mathrm{A}}^{\text {liq }}-n_{\mathrm{A}}^{\text {vap }} n_{\mathrm{B}}^{\text {liq }}\right)}{n_{\mathrm{A}}^{\text {vap }} v^{\text {liq }}-n_{\mathrm{A}}^{\text {liq }} v^{\text {vap }}} \mathrm{d} \mu_{\mathrm{B}} \\
& -\frac{[S]^{1 / \mathrm{v}}\left(n_{\mathrm{A}}^{\text {vap }} v^{\text {liq }}-n_{\mathrm{A}}^{\text {liq }} v^{\text {vap }}\right)-\left[N_{\mathrm{A}}\right]^{1 / \mathrm{v}}\left(s^{\text {vap }} v^{\text {liq }}-v^{\text {vap }} s^{\text {liq }}\right)+[V]^{l / v}\left(s^{\text {vap }} n_{\mathrm{A}}^{\text {liq }}-n_{\mathrm{A}}^{\text {vap }} s^{\text {liq }}\right)}{n_{\mathrm{A}}^{\text {vap }} v^{\text {liq }}-n_{\mathrm{A}}^{\text {liq }} v^{\text {vap }}} \mathrm{d} T \\
& =\frac{\operatorname{Det}\left(\begin{array}{ccc}
{\left[N_{\mathrm{B}}\right]^{1 / \mathrm{v}}} & {\left[N_{\mathrm{A}}\right]^{1 / \mathrm{v}}} & {[V]^{1 / \mathrm{v}}} \\
n_{\mathrm{B}}^{\text {vap }} & n_{\mathrm{A}}^{\text {vap }} & v^{\text {vap }} \\
n_{\mathrm{B}}^{\text {liq }} & n_{\mathrm{A}}^{\text {liq }} & v^{\text {liq }}
\end{array}\right)}{\operatorname{Det}\left(\begin{array}{cc}
n_{\mathrm{A}}^{\text {vap }} & v^{\text {vap }} \\
n_{\mathrm{A}}^{\text {liq }} & v^{\text {liq }}
\end{array}\right)} \mathrm{d} \mu_{\mathrm{B}}-\frac{\operatorname{Det}\left(\begin{array}{ccc}
{[S]^{1 / \mathrm{v}}} & {\left[N_{\mathrm{A}}\right]^{1 / \mathrm{v}}} & {[V]^{1 / \mathrm{v}}} \\
s^{\text {vap }} & n_{\mathrm{A}}^{\text {vap }} & v^{\text {vap }} \\
s^{\text {liq }} & n_{\mathrm{A}}^{\text {liq }} & v^{\text {liq }}
\end{array}\right)}{\operatorname{Det}\left(\begin{array}{cc}
n_{\mathrm{A}}^{\text {vap }} & v^{\text {vap }} \\
n_{\mathrm{A}}^{\text {liq }} & v^{\text {liq }}
\end{array}\right)} \mathrm{d} T
\end{aligned}
$$

on the orientation of the grain boundary with respect to the crystal.
The term multiplying $\mathrm{d} \mu_{\mathrm{B}}$ is the right-hand side of the traditional form of the Gibbs' adsorption isotherm. In the 
notation defined in Eq. (39), and in Equation 514 of Gibbs' "Equilibrium of Heterogeneous Substances" [184], this becomes $\mathrm{d} \gamma^{1 / \mathrm{v}}=-\Gamma_{\mathrm{B}(\mathrm{A})} \mathrm{d} \mu_{\mathrm{B}}$ in the case that $\mathrm{d} T=0$ (the adsorption isotherm); in this form, the equations are derived by locating the dividing surface such that $\left[N_{\mathrm{A}}\right]^{1 / \mathrm{v}}=\left[V_{\mathrm{A}}\right]^{1 / \mathrm{v}}=0$. The equations derived above by elimination of variables do not depend on this particular choice of location, but it can be seen that this reduces to the $-\Gamma_{\mathrm{B}(\mathrm{A})}$ form when $\left[N_{\mathrm{A}}\right]^{1 / \mathrm{v}}$ and $[V]^{1 / \mathrm{v}}$ are set to zero:

$\Gamma_{\mathrm{B}(\mathrm{A})}=\frac{\left[N_{\mathrm{B}}\right]^{\mathrm{l|v}}\left(n_{\mathrm{A}}^{\text {liq }} v^{\text {vap }}-n_{\mathrm{A}}^{\text {vap }} v^{\text {liq }}\right)}{n_{\mathrm{A}}^{\text {liq }} v^{\text {vap }}-n_{\mathrm{A}}^{\text {vap }} v^{\text {liq }}}=\left[N_{\mathrm{B}}\right]^{1 / \mathrm{v}}$

$\alpha$-Crystal/ $\beta$-crystal, ternary alloy (A-B-C)

There are two Gibbs-Duhem equations (one for each of the $\alpha$ and $\beta$ phase) - each of which have three componentsand an anisotropic interface equation with orientation and misorientation dependence.

Above, we have eliminated $\mathrm{d} P$ and $\mathrm{d} \mu_{\mathrm{A}}$ in each case. The choice of eliminated variables is arbitrary and the results will be identical for any choice. However, it is convenient to eliminate variables such that the ones that remain are those which the experimentalist may wish to change. For example, suppose that we change $\mathrm{d} \mu_{\mathrm{A}}, \mathrm{d} P$, and $\mathrm{d} T$ independently and allow the $\mathrm{d} \mu_{\mathrm{B}}$ and $\mathrm{d} \mu_{\mathrm{C}}$ to change so as to maintain equilibrium between the two phases. In this case, we would eliminate $\mathrm{d} \mu_{\mathrm{B}}$ and $\mathrm{d} \mu_{\mathrm{C}}$ from the GibbsDuhem equations and obtain:

$$
\begin{aligned}
& \hat{n}^{0_{x}^{\alpha} \mid 0_{y}^{\beta}} \cdot \mathrm{d} \vec{\xi}=\frac{\operatorname{Det}\left(\begin{array}{ccc}
{\left[N_{\mathrm{A}}\right]_{x}^{\alpha_{x}^{\alpha}} 0_{y}^{\beta}} & {\left[N_{\mathrm{B}}\right]^{0_{x}^{\alpha} \mid 0_{y}^{\beta}}} & {\left[N_{\mathrm{C}}\right]^{0_{x}^{\alpha} \mid 0_{y}^{\beta}}} \\
n_{\mathrm{A}}^{\alpha} & n_{\mathrm{B}}^{\alpha} & n_{\mathrm{B}}^{\beta} \\
n_{\mathrm{A}}^{\beta} & n_{\mathrm{C}}^{\alpha} & n_{\mathrm{C}}^{\beta}
\end{array}\right)}{\operatorname{Det}\left(\begin{array}{cc}
n_{\mathrm{B}}^{\alpha} & n_{\mathrm{B}}^{\beta} \\
n_{\mathrm{C}}^{\alpha} & n_{\mathrm{C}}^{\beta}
\end{array}\right)} \mathrm{d} \mu_{\mathrm{A}} \\
& -\frac{\operatorname{Det}\left(\begin{array}{ccc}
{[S]_{x}^{0_{x}^{\alpha} \mid 0_{y}^{\beta}}} & {\left[N_{\mathrm{B}}\right]_{x}^{0_{x}^{\alpha} \mid 0_{y}^{\beta}}} & {\left[N_{\mathrm{C}}\right]_{x}^{0_{x}^{\alpha} \mid 0_{y}^{\beta}}} \\
s^{\alpha} & n_{\mathrm{B}}^{\alpha} & n_{\mathrm{B}}^{\beta} \\
s^{\beta} & n_{\mathrm{C}}^{\alpha} & n_{\mathrm{C}}^{\beta}
\end{array}\right)}{\operatorname{Det}\left(\begin{array}{cc}
n_{\mathrm{B}}^{\alpha} & n_{\mathrm{B}}^{\beta} \\
n_{\mathrm{C}}^{\alpha} & n_{\mathrm{C}}^{\beta}
\end{array}\right)} \mathrm{d} T \\
& +\frac{\operatorname{Det}\left(\begin{array}{ccc}
{[V]^{0_{x}^{\alpha} \mid 0_{y}^{\beta}}} & {\left[N_{\mathrm{B}}\right]^{0_{x}^{\alpha} \mid 0_{y}^{\beta}}} & {\left[N_{C}\right]_{x}^{0_{x}^{\alpha} \mid 00_{y}^{\beta}}} \\
v^{\alpha} & n_{\mathrm{B}}^{\alpha} & n_{B}^{\beta} \\
v^{\beta} & n_{\mathrm{C}}^{\alpha} & n_{C}^{\beta}
\end{array}\right)}{\operatorname{Det}\left(\begin{array}{cc}
n_{B}^{\alpha} & n_{B}^{\beta} \\
n_{C}^{\alpha} & n_{C}^{\beta}
\end{array}\right)} \mathrm{d} P
\end{aligned}
$$

\section{General case: Cahn's determinants}

In the sequence of examples given above, the coefficients which multiply the differential quantities become more lengthy as the system becomes more general. However, the form of the last example where the coefficients are written as a ratio of determinants indicates the possibility of a generalization to any case. The ratios are the determinants

\begin{tabular}{|c|c|}
\hline Adsorbate & $\begin{array}{l}\text { A component which belongs to an adsorption layer } \\
\text { (often used when the species which are in this } \\
\text { adsorption layer come from the vapor phase) }\end{array}$ \\
\hline Adsorption & $\begin{array}{l}\text { Positive or negative excess (atoms or moles) of a } \\
\text { component, per unit area of interface, in a system } \\
\text { with an interface in comparison with a system } \\
\text { consisting just of the abutting bulk phases }\end{array}$ \\
\hline $\begin{array}{r}\text { Bad/poor } \\
\text { wetting }\end{array}$ & $\begin{array}{l}\text { Traditionally used to describe a contact angle } \\
\text { greater than } 90^{\circ}\end{array}$ \\
\hline $\begin{array}{l}\text { Capillary } \\
\text { length }\end{array}$ & $\begin{array}{l}\text { The length defined by the balance of capillary and } \\
\text { hydrostatic pressures }\end{array}$ \\
\hline $\begin{array}{c}\text { Complete } \\
\text { wetting }\end{array}$ & $\begin{array}{l}\text { A zero contact or dihedral angle of a phase at an } \\
\text { interface }\end{array}$ \\
\hline Complexions & $\begin{array}{l}\text { Equilibrium } 2 \mathrm{D} \text { state at an interface, characterized } \\
\text { by an interfacial excess of chemical components } \\
\text { and/or structural order parameters, at prescribed } \\
\text { chemical potentials }\end{array}$ \\
\hline $\begin{array}{l}\text { Critical point } \\
\text { wetting }\end{array}$ & $\begin{array}{l}\text { A term introduced by Cahn [127] to describe a } \\
\text { transition from partial wetting to complete wetting } \\
\text { as a bulk critical point is approached. For example, } \\
\text { when two liquids coexist within a miscibility gap } \\
\text { with an external third phase like a gas or a solid } \\
\text { wall, and are heated towards the critical point, both } \\
\text { the difference between their interfacial energies } \\
\text { with the third phase, as well as the energy of the } \\
\text { liquid-liquid interface separating them, vanish. } \\
\text { Since the difference between the interfacial } \\
\text { energies of the two liquids with the third phase } \\
\text { vanishes faster than the liquid-liquid interfacial } \\
\text { energy, complete wetting of one of the two liquid } \\
\text { in contact with the third phase on the other liquid } \\
\text { arises before the bulk critical point is reached }\end{array}$ \\
\hline Dewetting & $\begin{array}{l}\text { The kinetic process through which a phase (in the } \\
\text { form of a film) breaks up or agglomerates into } \\
\text { droplets (or particles in the solid state) because } \\
\text { wetting is not complete and/or the film is too thin }\end{array}$ \\
\hline $\begin{array}{l}\text { Diffuse } \\
\text { interface }\end{array}$ & $\begin{array}{l}\text { Cahn's description of an interface between two } \\
\text { phases of a miscibility gap which are at } \\
\text { equilibrium; the gradients of "intensive } \\
\text { interfacial parameters" are spread along a weak } \\
\text { gradient [185] }\end{array}$ \\
\hline $\begin{array}{l}\text { Disjoining } \\
\text { pressure }\end{array}$ & $\begin{array}{l}\text { The pressure that must be applied to two interfaces to } \\
\text { maintain a given separation distance }\end{array}$ \\
\hline
\end{tabular}
derived by Cahn using Cramer's rule.

\section{Panel 6: nomenclature}




\begin{tabular}{|c|c|c|c|}
\hline \multirow{3}{*}{$\begin{array}{l}\text { Dry versus moist } \\
\text { interface }\end{array}$} & \multirow{3}{*}{$\begin{array}{l}\text { A "dry" interface has been used to describe } \\
\text { an interface with no chemical excess } \\
\text { (which probably never exists). A "moist" } \\
\text { interface has been used to describe an } \\
\text { interface with a finite amount of } \\
\text { adsorbate (a specific complexion) }\end{array}$} & Premelting-like & Same as premelting \\
\hline & & Prewetting & $\begin{array}{l}\text { A first-order complexion transition at an } \\
\text { interface; for example transition in } \\
\text { adsorption or interfacial order parameter }\end{array}$ \\
\hline & & \multirow[t]{3}{*}{ Prewetting film } & \multirow{3}{*}{$\begin{array}{l}\text { This term is used in the literature to describe } \\
\text { an interfacial state (complexion), where } \\
\text { complete wetting by a new phase is } \\
\text { preceded by a film with a composition very } \\
\text { close to that of the new phase as } \\
\text { coexistence is approached. The new phase } \\
\text { will form when coexistence is reached }\end{array}$} \\
\hline Frank-van der Merwe & $\begin{array}{l}\text { A mode of thin film growth (layer-by- } \\
\text { layer); this is not complete wetting } \\
\text { because there is no equilibrium between } \\
\text { the film and the substrate }\end{array}$ & & \\
\hline \multirow[t]{2}{*}{ GB-disordering } & \multirow{2}{*}{$\begin{array}{l}\text { Transition from an ordered to a disordered } \\
\text { state of a complexion at a grain boundary }\end{array}$} & & \\
\hline & & \multirow{3}{*}{$\begin{array}{l}\text { Pseudo-partial } \\
\text { wetting }\end{array}$} & \multirow{3}{*}{$\begin{array}{l}\text { This term occasionally appears in the } \\
\text { literature to describe a partial wetting } \\
\text { situation of a drop where an adsorption } \\
\text { layer of undetermined thickness of the } \\
\text { components of the drop, spreads ahead of } \\
\text { the triple line on the solid substrate }\end{array}$} \\
\hline Good wetting & $\begin{array}{l}\text { Traditionally used to describe a contact } \\
\text { angle less than } 90^{\circ}\end{array}$ & & \\
\hline \multirow[t]{3}{*}{ Interface/surface phase } & \multirow{3}{*}{$\begin{array}{l}\text { An incorrect terminology used in the past to } \\
\text { address complexions. Phases can certainly } \\
\text { form at interfaces or surfaces, but these } \\
\text { are necessarily 3D phases which must } \\
\text { appear on the phase diagram of the } \\
\text { corresponding system, and are not } \\
\text { complexions }\end{array}$} & & \\
\hline & & Quasi-liquid film & Same as premelted film \\
\hline & & Surficial film & $\begin{array}{l}\text { This is the surface equivalent of an } \\
\text { intergranular film. It has most often been } \\
\text { used to describe an adsorption film (a }\end{array}$ \\
\hline \multirow[t]{2}{*}{$\begin{array}{l}\text { Interface/surface } \\
\text { reconstruction }\end{array}$} & \multirow{2}{*}{$\begin{array}{l}\text { A structural change of the interfacial region, } \\
\text { leading to a new } 2 \mathrm{D} \text { unit cell. The thickness } \\
\text { of the cell may not be limited to a single } \\
\text { monolayer. An example is the } 7 \times 7 \\
\text { reconstruction on the (111) surface of } \mathrm{Si}\end{array}$} & & $\begin{array}{l}\text { complexion) at a surface. In some cases this } \\
\text { has also been used to describe a film of a } \\
\text { wetting phase at a surface }\end{array}$ \\
\hline & & Segregation & $\begin{array}{l}\text { In the realm of thermodynamics and } \\
\text { equilibrium, this is equivalent to adsorption. }\end{array}$ \\
\hline \multirow[t]{4}{*}{$\begin{array}{l}\text { Intergranular Films } \\
\text { (IGFs) }\end{array}$} & \multirow{4}{*}{$\begin{array}{l}\text { Interfacial regions of constant thickness (at } \\
\text { fixed chemical potentials) between two } \\
\text { grains of the same phase with a defined } \\
\text { misorientation, or between two grains of } \\
\text { different phases. Due to the typical } \\
1-2 \mathrm{~nm} \text { thickness of some IGFs at grain } \\
\text { boundaries in ceramics, IGFs were } \\
\text { originally referred to as "phases" or } \\
\text { wetting films, although they have now } \\
\text { been clearly shown to be complexions. } \\
\text { Equivalent terms used in the literature } \\
\text { include equilibrium films, equilibrium } \\
\text { amorphous films (although complexions } \\
\text { are not necessarily amorphous), } \\
\text { multilayer adsorbates, and wetting films, } \\
\text { although adsorbed layers are clearly not } \\
\text { wetting films }\end{array}$} & & $\begin{array}{l}\text { However, it is also unfortunately used by } \\
\text { many to indicate the diffusion of species } \\
\text { towards an interface driven by equilibration } \\
\text { processes. "Enrichment" is probably a } \\
\text { better term for this situation }\end{array}$ \\
\hline & & Spreading & $\begin{array}{l}\text { The kinetics of triple line displacement of a } \\
\text { liquid on a substrate in the presence of a } \\
\text { fluid phase }\end{array}$ \\
\hline & & Stranski-Krastanov & $\begin{array}{l}\text { A mode of thin film growth (islands on top of } \\
\text { an adsorption layer); this is not a wetting } \\
\text { state because there is no equilibrium } \\
\text { between the film and the substrate. }\end{array}$ \\
\hline & & $\begin{array}{l}\text { Submonolayer } \\
\text { adsorbate }\end{array}$ & $\begin{array}{l}\text { An amount of excess which, if projected onto } \\
\text { one atomic plane and assumed to occupy } \\
\text { specific lattice sites, occupies less than a } \\
\text { complete monolayer of lattice sites }\end{array}$ \\
\hline \multirow[t]{3}{*}{$\begin{array}{l}\text { Interface layering/ } \\
\text { layering transition }\end{array}$} & \multirow{3}{*}{$\begin{array}{l}\text { First (or higher) order adsorption transitions } \\
\text { from one layer to the next; the thickness } \\
\text { of layers depends on the range of } \\
\text { interaction (one atom thick for metals, } \\
\text { several ions thick in iono-covalent } \\
\text { materials) }\end{array}$} & $\begin{array}{l}\text { Surface/interface } \\
\text { freezing }\end{array}$ & $\begin{array}{l}\text { Ordering transition of a liquid surface state } \\
\text { from static and dynamic disorder to ordered }\end{array}$ \\
\hline & & $\begin{array}{l}\text { Surface/interface } \\
\text { melting }\end{array}$ & Same as premelting \\
\hline & & \multirow[t]{2}{*}{ Volmer-Weber } & \multirow{2}{*}{$\begin{array}{l}\text { A mode of thin film growth (islands); this is not } \\
\text { a partial wetting state because there is no } \\
\text { equilibrium between the film and the substrate }\end{array}$} \\
\hline Liquid-like GB & A premelted grain boundary & & \\
\hline \multirow[t]{2}{*}{ Nano-wetting } & $\begin{array}{l}\text { An unfortunate and inappropriate use of } \\
\text { jargon to describe adsorption }\end{array}$ & \multirow[t]{4}{*}{$\begin{array}{l}\text { Wetting (transition) } \\
\text { temperature }\end{array}$} & \multirow{4}{*}{$\begin{array}{l}\text { Temperature at which a transition occurs from } \\
\text { partial to complete wetting. Since any } \\
\text { interfacial energy varies continuously as a } \\
\text { function of any intensive variable like } \\
\text { temperature or chemical potential, the contact } \\
\text { angle goes to zero continuously, while the } \\
\text { transition can be critical or first order }\end{array}$} \\
\hline & $\begin{array}{l}\text { A wetting state characterized by a contact } \\
\text { angle above zero and less than } 180^{\circ}\end{array}$ & & \\
\hline Perfect wetting & Same as complete wetting & & \\
\hline \multirow[t]{2}{*}{ Premelting } & \multirow{2}{*}{$\begin{array}{l}\text { An interfacial state transition from an } \\
\text { ordered state to a state which displays } \\
\text { static and dynamic disorder, as in a liquid, } \\
\text { but below the bulk melting point. This } \\
\text { means that diffusion rates within this } \\
\text { region are similar to those in a liquid }\end{array}$} & & \\
\hline & & Work of adhesion & $\begin{array}{l}\text { The reversible thermodynamic work to create } \\
\text { two surfaces of two different phases at } \\
\text { equilibrium, from an equilibrated interface } \\
\text { between the two phases }\end{array}$ \\
\hline
\end{tabular}




\begin{tabular}{ll}
\hline $\begin{array}{l}\text { Work of } \\
\text { separation }\end{array}$ & $\begin{array}{l}\text { Irreversible work to create two non-equilibrated } \\
\text { surfaces of two different phases from an } \\
\text { equilibrated interface between the two phases. It } \\
\text { will always be larger than the work of adhesion }\end{array}$ \\
\hline
\end{tabular}

\section{Summary}

High temperature capillarity is an important scientific and technological field of research, which covers both thermodynamics (wetting) and kinetics (spreading). In writing this review, we have first attempted to identify phenomena related to wetting, and then proceeded to describe how these phenomena may be modified by the presence of adsorption. We have also emphasized that the simple measure of a contact (or dihedral) angle is not sufficient to understand wetting, and that the anisotropy of crystalline materials can play an important role in the minimization of interface and surface energy. Furthermore, we have summarized the strong influence that chemical heterogeneity and roughness of surfaces can have on both wetting and spreading, since they impact the motion of the triple line.

We have also reviewed wetting between two solid phases, since while solid-liquid interfaces are often important for materials processing, it is the solid-solid interface which most often determines the properties of the final material system. Solid-solid interfacial energy can be experimentally measured, and allows determination of the important value of the thermodynamic work of adhesion; but once again the anisotropy of crystalline materials must be taken into account.

Finally, we have reviewed the fundamentals of adsorption at the thermodynamic, or macroscopic, scale, and how adsorption may play a decisive role in both wetting and wetting transitions. Adsorption has also been considered at the level of the local atomic structure, first with regard to excess distribution and then by using the concept of interface complexions. It is our hope that this review will convince the reader that wetting phenomena cannot be addressed without considering complexions and/or complexion transitions, and this important concept provides a bridge to future work aimed at merging continuum and atomistic approaches to interface science.

Acknowledgements We would like to thank our students who critically reviewed this paper: R. Zucker and G. Atiya. We thank R. Zucker for contributing to Panel 2. We also acknowledge the staff of the Best Western in Cassis, France, who showed unending patience when we descended upon their lobby for an entire week to consolidate this review. The effort leading to this review received funding from the European Community's Seventh Framework Programme (FP7/ 2007-2013) under grant agreement FP7-NMP-2009-CSA-233484MACAN (Merging Atomistic and Continuum Analysis of Nanometer Length-Scale Metal-Oxide Systems for Energy and Catalysis Applications).
Open Access This article is distributed under the terms of the Creative Commons Attribution License which permits any use, distribution, and reproduction in any medium, provided the original author(s) and the source are credited.

\section{References}

1. Akselsen OM (1992) J Mater Sci 27:1989. doi:10.1007/ BF01117909

2. Marks RA, Sugar JD, Glaeser AM (2001) J Mater Sci 36:5609. doi:10.1023/A:1012565600601

3. Yacobi BG, Martin S, Davis K, Hudson A, Hubert M (2002) J Appl Phys 91:6227

4. Kim JY, Hardy JS, Scott Weil K (2005) J Am Ceram Soc $88: 2521$

5. Karpel A, Gur G, Atzmon Z, Kaplan WD (2007) J Mater Sci 42:2334. doi:10.1007/s10853-007-1592-Z

6. Karpel A, Gur G, Atzmon Z, Kaplan WD (2007) J Mater Sci 42:2347. doi:10.1007/s10853-007-1593-y

7. Hoyt JJ, Asta M, Haxhimali T, Karma A, Napolitano RE, Trivedi R, Laird BB, Morris JR (2004) MRS Bull 29:935

8. Kelton KF, Greer AL, Herlach DM, Holland-Moritz D (2004) MRS Bull 29:940

9. Levi G, Kaplan WD, Bamberger M (2002) Mater Sci Eng, A 326:288

10. Loehman RE, Ewsuk K, Tomsia AP (1996) J Am Ceram Soc 79:27

11. Kennedy AR, Wood JD, Weager BM (2000) J Mater Sci 35:2909. doi:10.1023/A:1004714407371

12. Ji Y, Yeomans JA (2002) J Mater Sci 37:5229. doi:10.1023/A: 1021000318894

13. Aizenshtein M, Frage N, Froumin N, Shapiro-Tsoref E, Dariel MP (2006) J Mater Sci 41:5185. doi:10.1007/s10853-0060436-6

14. Wagner F, Garcia DE, Krupp A, Claussen N (1999) J Eur Ceram Soc 19:2449

15. Voue M, De Coninck J (2000) Acta Mater 48:4405

16. Muolo ML, Ferrera E, Morbelli L, Passerone A (2004) Scripta Mater 50:325

17. Laurent V, Rado C, Eustathopoulos N (1996) Mater Sci Eng, A 205:1

18. Cook RF (1990) Acta Metall Mater 38:1083

19. Degraef M, Dalgleish BJ, Turner MR, Evans AG (1992) Acta Metall Mater 40:S333

20. Dalgleish BJ, Saiz E, Tomsia AP, Cannon RM, Ritchie RO (1994) Scr Metall Mater 31:1109

21. Saiz E, Tomsia AP, Suganuma K (2003) J Eur Ceram Soc 23:2787

22. Gremillard L, Saiz E, Chevalier J, Tomsia AP (2004) Zeitschrift Fur Metallkunde 95:261

23. Cahn JW (1979) In: Johnson WC, Blakely JM (eds) Interfacial segregation. ASM, Metals Park, p 3

24. Cannon RM, Carter WC (1989) J Am Ceram Soc 72:1550

25. Carter WC (1988) Acta Metall 36(8):2283

26. Marmur A (2012) Soft Matter 8(26): 2867

27. Johnson RE, Dettre RH (1964) In: Fowkes FM (ed) Contact angle, wettability, and adhesion. Advances in chemistry series, vol 43. American Chemical Society, Washington, DC, p 112

28. Chatain D, Ghetta V, Wynblatt P (2004) Interface Sci 12(1):7

29. Cheng WC, Wynblatt P (1997) J Cryst Growth 173(3-4):513

30. De Gennes PG (1985) Rev Mod Phys 57(3):827

31. Huh C, Mason SG (1977) J Colloid Interface Sci 60(1):11

32. Wenzel RN (1936) Ind Eng Chem 28:988 
33. Eick JD, Good RJ, Neumann AW (1975) J Colloid Interface Sci 53(2): 235

34. Cassie ABD, Baxter S (1948) Discuss Faraday Soc 3:11

35. De Jonghe V, Chatain D (1995) Acta Metall Mater 43:1505

36. Chatain D, Lesueur C, Baland JP (2006) Langmuir 22(9):4230

37. Chatain D, Lewis D, Baland JP, Carter WC (2006) Langmuir 22(9):4237

38. Naidich YV, Voitovich RP, Zabuga VV (1995) J Colloid Interface Sci 174(1):104

39. Pompe T, Herminghaus S (2000) Phys Rev Lett 85(9):1930

40. Solomentsev Y, White LR (1999) J Colloid Interface Sci 218: 122

41. Mullins WW (1963) Solid surface morphologies governed by capillarity. In: Robertson WD, Gjostein NA (eds) Metal surfaces: structure, energetics and kinetics. ASM, Cleveland, p 17

42. Wulff G (1901) Zeitschrift fur Krystallographie und Mineralogie 34(5/6):449

43. Chatain D (2008) Annu Rev Mater Res 38:45

44. Herring C (1951) Phys Rev 82(1):87

45. Lipkin DM, Clarke DR, Evans AG (1998) Acta Mater 46(13): 4835

46. Winterbottom WL (1967) Acta Metall 15(2):303

47. Lee JK, Aaronson HI, Russell KC (1975) Surf Sci 51:302

48. Frank FC (1963) In: Mullins WW (ed) Metal surfaces. American Society for Metals, Metals Park, p 1

49. Cahn JW, Carter WC (1996) Metall Mater Trans A 27(6):1431

50. Taylor JE (1992) Acta Metall Mater 40(7):1475

51. Pilliar RM, Nutting J (1967) Philos Mag 16(139):181

52. Heyraud JC, Metois JJ (1980) Acta Metall 28(12):1789

53. Silly F, Castell MR (2005) Appl Phys Lett 87:053106

54. Hansen KH, Worren T, Stempel S, Laegsgaard E, Baumer M, Freund HJ, Besenbacher F, Stensgaard I (1999) Phys Rev Lett 83(20):4120

55. Cahn JW, Hoffman DW (1974) Acta Metall 22(10):1205

56. Levi G, Kaplan WD (2003) Acta Mater 51(10):2793

57. Thangadurai P, Lumelsky Y, Silverstein MS, Kaplan WD (2008) Mater Charact 59(11):1623

58. Sadan H, Kaplan WD (2006) J Mater Sci 41(16):5099. doi: 10.1007/s10853-006-0437-5

59. Baram M, Kaplan WD (2008) J Microsc 232(3):395

60. Meltzman H, Kauffmann Y, Thangadurai P, Drozdov M, Baram M, Brandon D, Kaplan WD (2009) J Microsc 236(3):165

61. Meltzman H, Chatain D, Avizemer D, Besmann TM, Kaplan WD (2011) Acta Mater 59(9):3473

62. Meltzman H, Mordehai D, Kaplan WD (2012) Acta Mater 60(11):4359

63. Nussbaum E, Meltzman H, Kaplan WD (2012) J Mater Sci 47(4):1647. doi:10.1007/s10853-011-5707-1

64. Goldfarb I, Grossman S, Cohen-Taguri G, Levinshtein M (2004) Appl Surf Sci 238(1-4):29

65. Goldfarb I, Cohen-Taguri G, Grossman S, Levinshtein M (2005) Phys Rev B 72(7):075430

66. Muller P, Kern R (2000) Surf Sci 457(1-2):229

67. Irawan T, Barke I, Hovel H (2005) Appl Phys A 80:929

68. Saylor DM, Rohrer GS (1999) J Am Ceram Soc 82(6):1529

69. Lee JK, Aaronson HI (1975) Acta Metall 23:799

70. Lee JK, Aaronson HI (1975) Acta Metall 23:809

71. Johnson E, Johansen A, Dahmen U, Chen S, Fujii T (2001) Mater Sci Eng A 304:187

72. Hamilton JC, Leonard F, Johnson E, Dahmen U (2007) Phys Rev Lett 98(23):236102

73. Siem EJ, Carter WC, Chatain D (2004) Philos Mag 84(10):991

74. Siem EJ, Carter WC (2005) J Mater Sci 40(12):3107. doi: 10.1007/s10853-005-2671-7

75. Zucker RV, Chatain D, Dahmen U, Hagège S, Carter WC (2012) J Mater Sci 47(24):8290. doi:10.1007/s10853-012-6739-x
76. Saiz E, Cannon RM, Tomsia AP (2000) Acta Mater 48(18-19): 4449

77. Chatain D, Carter WC (2004) Nat Mater 3(12):843

78. Ghetta V, Chatain D (2002) J Am Ceram Soc 85(4):961

79. Nichols FA, Mullins WW (1965) Trans Metall Soc AIME 233(10): 1840

80. Levi G, Kaplan WD (2003) Acta Mater 51(10):2793

81. Thompson CV (2012) Annu Rev Mater Res 42:399

82. Srolovitz DJ, Safran SA (1986) J Appl Phys 60(1):247

83. Srolovitz DJ, Goldiner MG (1995) J Miner Met Mater Soc 47(3):31

84. Muller CM, Spolenak R (2010) Acta Mater 58(18):6035

85. Ye J, Thompson CV (2011) Acta Mater 59(2):582

86. Klinger L, Amram D, Rabkin E (2011) Scripta Mater 64(10):962

87. Genin FY, Mullins WW, Wynblatt P (1992) Acta Metall Mater 40(12):3239

88. Genin FY, Mullins WW, Wynblatt P (1994) Acta Metall Mater 42(4): 1489

89. Khenner M (2008) Phys Rev B 77(24):245445

90. Khenner M (2008) Phys Rev B 77(16):165414

91. Schmirgeld-Mignot L, Molinas-Mata PJA, Poissonnet S, Martin G (2000) Philos Mag Lett 80(1):33

92. Shaffir E, Riess I, Kaplan WD (2009) Acta Mater 57(1):248

93. Chatain D, Wynblatt P, Rohrer GS (2005) Acta Mater 53(15): 4057

94. Curiotto S, Chien H, Meltzman H, Wynblatt P, Rohrer GS, Kaplan WD, Chatain D (2011) Acta Mater 59(13):5320

95. Beszeda I, Beke DL (1997) Appl Phys A 64(6):583

96. Beszeda I, Kocsis T, Imreh G, Weigl F, Boyen HG, Ziemann P, Beke DL (2006) Appl Surf Sci 253(3):1160

97. Amram D, Klinger L, Rabkin E (2012) Acta Mater 60(6-7): 3047

98. Ye J, Thompson CV (2011) Adv Mater 23(13):1567

99. Wang D, Schaaf P (2012) J Mater Sci 47(4):1605. doi: 10.1007/s10853-011-5716-0

100. Giermann AL, Thompson CV (2012) Appl Phys Lett 101(5): 051904

101. Ahn T-M, Tien JK, Wynblatt P (1980) J Catal 66(2):335

102. Hajjar S, Garreau G, Josien L, Bubendorff JL, Berling D, Mehdaoui A, Pirri C, Maroutian T, Renard C, Bouchier D, Petit M, Spiesser A, Dau MT, Michez L, Le Thanh V, Mentes TO, Nino MA, Locatelli A (2011) Phys Rev B 84(12):125325

103. Pina-Zapardiel R, Montero I, Esteban-Cubillo A, Moya JS, Kaplan WD, Paramasivam T, Pecharroman C (2011) J Nanopart Res 13(10):5239

104. Beckel D, Dubach A, Grundy AN, Infortuna A, Gauckler LJ (2007) J Eur Ceram Soc 28(1):49

105. Baumann N, Mutoro E, Janek J (2010) Solid State Ionics 181(1-2):7

106. Mikhelashvili V, Meyler B, Yoffis S, Salzman J, Garbrecht M, Cohen-Hyams T, Kaplan WD, Eisenstein G (2009) Appl Phys Lett 95(2):023104

107. Mikhelashvili V, Meyler B, Yofis S, Salzman J, Garbrecht M, Cohen-Hyams T, Kaplan WD, Eisenstein G (2010) J Electrochem Soc 157(4):H463

108. Mikhelashvili V, Shneider Y, Meyler B, Atiya G, Yofis S, Cohen-Hyams T, Kaplan WD, Lisiansky M, Roizin Y, Salzman J, Eisenstein G (2012) J Appl Phys 112(2):024319

109. Léger L, Joanny JF (1992) Rep Prog Phys 55(4):431

110. Brochard-Wyart F, Di Meglio JM, Quere D, de Gennes PG (1991) Langmuir 7(2):335

111. Tiberg F, Cazabat AM (1994) Langmuir 10(7):2301

112. Moon J, Lowekamp J, Wynblatt P, Garoff S, Suter RM (2001) Surf Sci 488(1-2):73

113. Monchoux JP, Chatain D, Wynblatt P (2007) Surf Sci 601(4): 1101 
114. Heine DR, Grest GS, Webb EB (2005) Phys Rev Lett 95(10): 107801

115. Saiz E, Tomsia AP (2004) Nat Mater 3:903

116. Blake TD (2006) J Colloid Interface Sci 299(1):1

117. Saiz E, Tomsia AP (2005) Curr Opin Solid State Mater Sci 9(4-5): 167

118. Saiz E, Benhassine M, De Coninck J, Tomsia AP (2010) Scripta Mater 62(12):934

119. Benhassine M, Saiz E, Tomsia AP, De Coninck J (2009) Langmuir 25(19):11450

120. Benhassine M, Saiz E, Tomsia AP, De Coninck J (2010) Acta Mater 58(6):2068

121. Benhassine M, Saiz E, Tomsia AP, De Coninck J (2011) Acta Mater 59(3):1087

122. Spaepen F (1975) Acta Metall 23:729

123. Abraham FF, Singh Y (1977) J Chem Phys 67(5):2384

124. Hashibon A, Adler J, Finnis MW, Kaplan WD (2001) Interface Sci 9:175

125. Hashibon A, Adler J, Finnis MW, Kaplan WD (2002) Comput Mater Sci 24(4):443

126. Buta D, Asta M, Hoyt JJ (2008) Phys Rev E 78(3):031605

127. Kyrlidis A, Brown RA (1995) Phys Rev E 51(6-A):5832

128. Kaplan WD, Kauffmann Y (2006) Annu Rev Mater Res 36(1):1

129. Kang J, Zhu J, Curtis C, Blake D, Glatzmaier G, Kim Y-H, Wei S-H (2012) Phys Rev Lett 108(22):226105

130. Huisman WJ, Peters JF, Zwanenburg MJ, de Vries SA, Derry TE, Abernathy D, van der Veen JF (1997) Nature 390:379

131. Donnelly SE, Birtcher RC, Allen CW, Morrison I, Furuya K, Song MH, Mitsuishi K, Dahmen U (2002) Science 296(5567): 507

132. Oh SH, Kauffmann Y, Scheu C, Kaplan WD, Ruhle M (2005) Science 310(5748):661

133. Oh SH, Chisholm MF, Kauffmann Y, Kaplan WD, Luo W, Rühle M, Scheu C (2010) Science 330:489

134. Yu C-J, Evmenenko G, Richter AG, Datta A, Kmetko J, Dutta P (2001) Appl Surf Sci 182(3-4):231

135. Reedijk MF, Arsic J, Hollander FFA, de Vries SA, Vlieg E (2003) Phys Rev Lett 90(6):066103

136. Gibbs JW (1961) The scientific papers of J. Willard Gibbs, vol 1. Dover, New York, p 219

137. Hirth JP (1965) In: Mueller WM (ed) Energetics in metallurgical phenomena, vol II. Gordon and Breach, New York, p 1

138. Hirth JP (1973) In: Shimodaiva S (ed) Structure and properties of metal surfaces, Honda Memorial Series in Materials Science No. 1. Maruzen Co. Ltd., Tokyo, p 10

139. Rowlinson JS, Widom B (1982) Molecular theory of capillarity. Clarendon Press, Oxford, p 25

140. Wynblatt P, Chatain D (2006) Metall Mater Trans A 37(9):2595

141. Defay R, Prigogine I, Bellmans A, Everett DH (1966) Surface tension and adsorption. Wiley, New York, pp 158

142. Hondros ED, Seah MP (1977) Metall Trans A 8(9):1363

143. Fowler RH, Guggenheim EA (1939) Statistical Thermodynamics. Macmillan, New York, p 429

144. McLean D (1957) Grain boundaries in metals. Oxford Press, London, p 116

145. Ono S, Kondo S (1960) In: Flugge S (ed) Encyclopedia of physics, vol 10. Springer, Berlin, p 134

146. Wynblatt P, Ku RC (1977) Surf Sci 65:511

147. Wynblatt P, Ku RC (1979) In: Johnson WC, Blakely JM (eds) Interfacial segregation. ASM, Metals Park, p 115
148. Friedel J (1954) Adv Phys 3:446

149. Wang Z, Wynblatt P (1998) Acta Mater 46(14):4853

150. Ghetta V, Fouletier J, Chatain D (1996) Acta Mater 44:1927

151. Saiz E, Cannon RM, Tomsia AP (2008) Annu Rev Mater Res 38:197

152. Wynblatt P, Curiotto S, Chatain D (2010) Surf Sci 604(17-18): 1369

153. Wynblatt P (2008) Annu Rev Mater Res 38:173

154. Cahn JW (1977) J Chem Phys 66:3667

155. Moldover MR, Cahn JW (1980) Science 207:1073

156. Schick M (1990) In: Charvolin J, Joanny JF, Zinn-Justin J (eds) Liquids at Interfaces. Elsevier, Amsterdam, p 415

157. Pandit R, Schick M, Wortis M (1982) Phys Rev B 26(9):5112

158. Wynblatt P, Saúl A, Chatain D (1998) Acta Mater 46(7):2337

159. Dillon SJ, Harmer MP (2007) Acta Mater 55(15):5247

160. Dillon SJ, Tang M, Carter WC, Harmer MP (2007) Acta Mater 55(18):6208

161. Tang M, Carter WC, Cannon RM (2006) Phys Rev B 73(2): 024102

162. Tang M, Carter WC, Cannon RM (2006) J Mater Sci 41(23):7691. doi:10.1007/s10853-006-0608-4

163. Tang M, Carter WC, Cannon RM (2006) Phys Rev Lett 97(7): 075502

164. Kauffmann Y, Oh SH, Koch CY, Hashibon A, Scheu C, Ruhle M, Kapaln WD (2011) Acta Mater 59(11):4378

165. Gandman M, Kauffmann Y, Koch CT, Kaplan WD (2013) Phys Rev Lett 110:086106

166. Baram M, Chatain D, Kaplan WD (2011) Science 332(6026): 206

167. Baram M, Garofalini SH, Kaplan WD (2011) Acta Mater 59(14): 5710

168. Metois JJ, Heyraud JC (2000) Surf Sci 446(3):L127

169. Moon J, Lowekamp J, Wynblatt P, Garoff S, Suter RM (2001) Surf Sci 488(1-2):73

170. Moon J, Wynblatt P, Garoff S, Suter RM (2004) Surf Sci $559(2-3): 15$

171. Monchoux JP, Chatain D, Wynblatt P (2006) Surf Sci 600(6): 1265

172. Chatain D, Wynblatt P, Ranguis A (2007) Surf Sci 601(6):1623

173. Kolpak AM, Li D, Shao R, Rappe AM, Bonnell DA (2008) Phys Rev Lett 101(3):036102

174. Cavallaro A, Burriel M, Roqueta J, Apostolidis A, Bernardi A, Tarancón A, Srinivasan R, Cook SN, Fraser HL, Kilner JA, McComb DW, Santiso J (2010) Solid State Ionics 181:592

175. Jud E, Tang M, Chiang YM (2008) J Appl Phys 103(11):114108

176. Chiang YM, Wang H, Lee JR (1998) J Microsc 191:275

177. Luo J, Cheng HK, Asl KM, Kiely CJ, Harmer MP (2011) Science 333(6050):1730

178. Dillon SJ, Harmer MP (2008) J Am Ceram Soc 91(7):2304

179. Dillon SJ, Harmer MP (2008) J Am Ceram Soc 91(7):2314

180. Gibbs JW (1876/1928) On the equilibrium of heterogeneous substances, vol 1. Collected works. Longmans, Green, and Co., New York

181. Smith CS (1952) Trans ASM 45:533

182. Gupta VK, Yoon DH, Meyer HH, Luo J (2007) Acta Mater 55(9):3131

183. Frolov T, Mishin Y (2012) Phys Rev B 85:224106

184. Gibbs WJ (1876) Trans Conn Acad Arts Sci III Oct. 1875-May 1876:108; May 1877-July 1878:343

185. Cahn JW, Hilliard JE (1958) J Chem Phys 28:258 